\title{
NONCONTACTING THERMOELECTRIC DETECTION OF MATERIAL IMPERFECTIONS IN METALS
}

\author{
Final Report \\ for Period September 15, 2000 - March 15, 2005
}

Peter B. Nagy (PI), Adnan H. Nayfeh (co-PI), Waseem I. Faidi, Hector Carreon, Balachander Lakshminaraya, Feng Yu, Bassam Abu-Nabah

\author{
Department of Aerospace Engineering and Engineering Mechanics \\ University of Cincinnati \\ Cincinnati, Ohio 45221-0070
}

May 2005

Prepared for

THE U.S. DEPARTMENT OF ENERGY

AWARD NO. DE-FG02-00ER45855 


\section{Summary}

This project was aimed at developing a new noncontacting thermoelectric method for nondestructive detection of material imperfections in metals. The method is based on magnetic sensing of local thermoelectric currents around imperfections when a temperature gradient is established throughout a conducting specimen by external heating and cooling. The surrounding intact material serves as the reference electrode therefore the detection sensitivity could be very high if a sufficiently sensitive magnetometer is used in the measurements. This self-referencing, noncontacting, nondestructive inspection technique offers the following distinct advantages over conventional methods: high sensitivity to subtle variations in material properties, unique insensitivity to the size, shape, and other geometrical features of the specimen, noncontacting nature with a substantial stand-off distance, and the ability to probe relatively deep into the material. The potential applications of this method cover a very wide range from detection metallic inclusions and segregations, inhomogeneities, and tight cracks to characterization of hardening, embrittlement, fatigue, texture, and residual stresses.

During the this project, we laid down the foundations of this new field of nondestructive materials characterization and made substantial advance towards all of the originally proposed research goals. We have successfully adapted the noncontacting thermoelectric method to a series of nondestructive materials characterization applications that are currently not accessible by any other known inspection method. In particular, we developed and experimentally verified a series of analytical models capable of predicting the thermoelectric signatures produced by inclusions of different material properties and geometrical features. In addition, we studied the feasibility of nondestructive detection and characterization of cracks and voids in textured polycrystalline materials, phase anomalies and anisotropic effects of the microstructure, and thermally induced residual stress relief in surface treated components. 


\section{CONTENT}

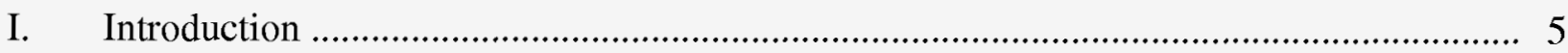

II. Modeling of the signal from isotropic spherical inclusions ........................................... 8

II.1 Infinite homogeneous medium containing a spherical inclusion .......................... 10

II.2 Infinite homogeneous medium containing a cylindrical inclusion .............. 13

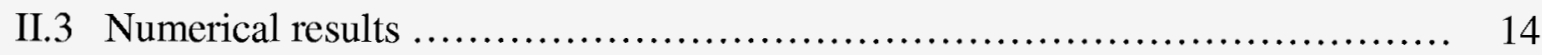

II.4 Half-space with a surface-breaking spherical inclusion ......................... 16

II.5 Half-space with a subsurface spherical inclusion ........................... 17

III Experimental investigation of the signal from isotropic spherical inclusions ............. 20

III.1 Predictions for a surface-breaking inclusion ............................... 20

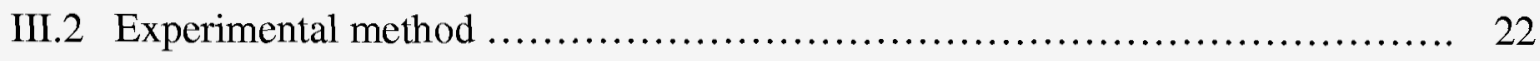

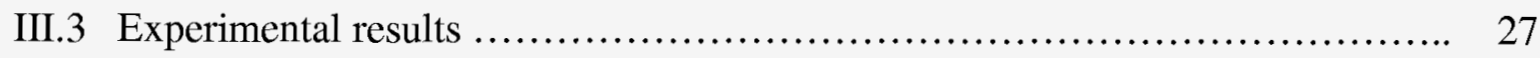

IV Theoretical modeling of the signal from cylindrical and ellipsoidal inclusions ............... 31

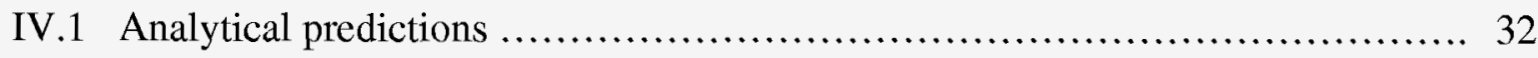

V Thermoelectric background signature produced by anisotropic materials ..................... 34

V.1 Analytical predictions ................................................... 36

V.2 Infinite dipole along a principal direction ................................... 41

V.3 Experimental verification .................................................. 43

VI Thermoelectric flaw signals produced by cracks and voids in anisotropic hosts ......... 46

VI.1 Analytical predictions .................................................. 47

VII Thermoelectric signature produced by material inhomogeneity .................................. 50

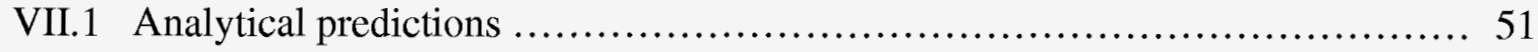

VII.2 Experimental arrangement f............................................. 55

VII.3 Experimental results in Ti-6Al-4V .................................... 58 
VII.4 Experimental results in brass

VIII Thermoelectric signature produced by applied stresses .......................................... 65

VIII.1 Experimental results .................................................... 68

IX Thermoelectric signature produced by residual stresses ........................................ 72

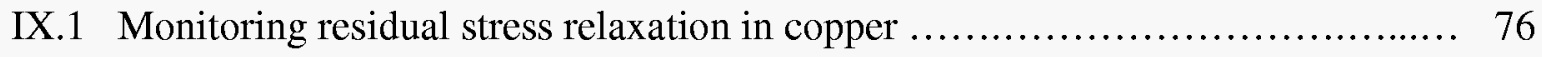

IX.2 Monitoring residual stress relaxation in nickel-base superalloys ............... 79

X Thermoelectric signals produced by material embrittlement .................................... 83

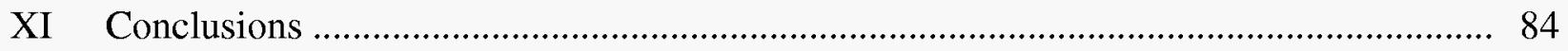

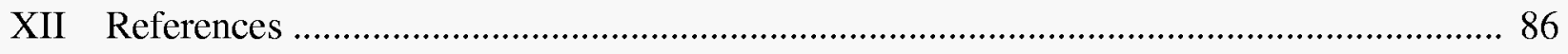

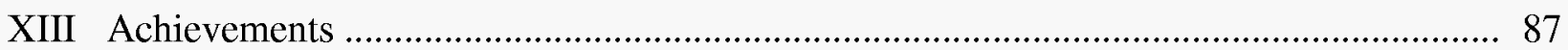




\section{Introduction}

A variety of different physical principles have been exploited for nondestructive detection, localization, and characterization of material imperfections in metals. The most popular techniques rely on ultrasonic, eddy current, x-ray radiographic, magnetic, thermal, and microwave principles. A common feature of these conventional methods is that they are sensitive to both intrinsic material (e.g., electrical and thermal conductivity, permeability, elastic stiffness, density, etc.) and spurious geometrical (e.g., size, shape, surface roughness, etc.) parameters. Unfortunately, these two classes of properties are often very difficult to separate, which sets the ultimate limit for the detectable smallest and/or weakest material imperfection. Conventional thermoelectric techniques, that have been used in nondestructive materials characterization for several decades, are essentially free from these geometrical limitations, i.e., they are sensitive to intrinsic material variations only regardless of the size, shape, and surface quality of the specimen to be tested. Essentially all existing thermoelectric NDT techniques are based on the well-known Seebeck effect that is commonly used in thermocouples to measure temperature at the junction of two different conductors. Ideally, regardless of the temperature difference between the junctions, only thermocouples made of different materials, or more precisely, materials of different thermoelectric power, will generate a thermoelectric signal. This unique feature makes the simple thermoelectric tester one of the most sensitive material discriminators used in nondestructive inspection. The thermoelectric power of metals is sensitive to a variety of material properties that can affect the measurement. Clearly, chemical composition exerts the strongest effect on the thermoelectric properties and accordingly the basic application of conventional thermoelectric materials characterization is metal sorting [1].

However, it is well known that under special conditions materials of identical chemical composition can also produce an efficient thermocouple as a result of different heat treatment, hardening, texture, residual stress, fatigue, etc., which can be further exploited for nondestructive testing of materials [2-5]. In spite of its obvious advantages over other methods, thermoelectric testing is rarely used in nondestructive testing (NDT) because of the requirement that a metallic contact be established between the specimen and the reference electrode. The resulting thermoelectric offset can be reduced, but not entirely eliminated, by decreasing the thermal and electrical resistance between the specimen and the reference electrode, e.g., via better cleaning or 
imposing higher contact pressure. Ultimately, the presence of this imperfect contact limits the detectability of small variations in material properties by the conventional thermoelectric technique [4]. Thermoelectric measurements are inherently relative in nature, therefore sensitive detection of subtle material variations requires that the thermoelectric power of the reference electrode be sufficiently similar to those of the intact material and the imperfection. In addition, very good metallic contact must exist between the reference electrodes and the part to be inspected. When either very small inclusions or very weak local variations such as texture, hardening, fatigue damage, or weak impurities are to be detected, the best sensitivity can be achieved by using the surrounding intact material as the reference electrode [5]. This so-called self-referencing method not only provides an ideal reference electrode, but also automatically eliminates the above mentioned spurious thermoelectric offset caused by having a less than perfect interface between the part to be tested and the surrounding intact reference material.

It was recently suggested that such self-referencing thermoelectric measurements can be also done in an entirely noncontact way by using high-sensitivity magnetometers to detect the weak thermoelectric currents around inclusions and other types of inhomogeneities, which occur when a temperature gradient exists in the specimen [6-8]. A schematic diagram of the selfreferencing thermoelectric method with noncontacting magnetic sensing is shown in Figure 1. External heating and/or cooling is applied to the specimen to produce a modest temperature gradient $\left(\approx 1{ }^{\circ} \mathrm{C} / \mathrm{cm}\right)$ in the region to be tested. As a result, different points on the boundary between the host material and the inclusion will be at different temperatures, therefore also at different thermoelectric potentials. These potential differences will produce opposite thermoelectric currents inside and outside the inclusion. The resulting thermoelectric currents form local loops that run in opposite directions on the two sides of the inclusion relative to the prevailing local heat flux, which can be detected by scanning the specimen with a sensitive magnetometer. Since the surrounding intact material serves as the "reference" electrode and there is no artificial interface between the host and the imperfect region, the detection sensitivity to minute variations in material properties can be extremely high. 


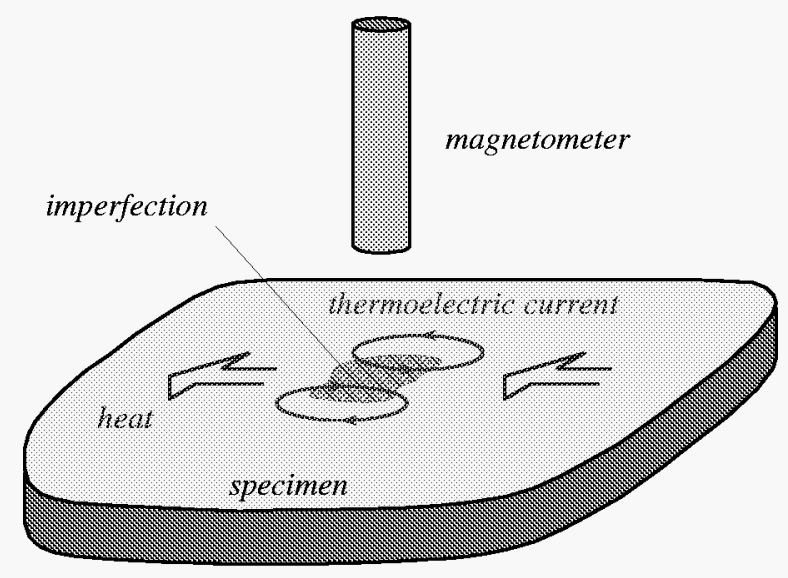

Figure 1 A schematic diagram of noncontacting thermoelectric detection of material imperfections by magnetic monitoring.

In this project, we demonstrated that the noncontacting thermoelectric technique can be used to detect various imperfections in metals, including foreign body inclusions and more subtle local property variations caused by service or manufacturing related effects such as cold work, localized texture, residual stress, heat damage, fatigue damage, fretting, etc. [9-16]. Like most other methods used in NDE, the detection sensitivity of the noncontacting thermoelectric method is ultimately limited by temporally coherent material noise rather than temporally incoherent electrical noise, which could be easily eliminated by simple time averaging. Strictly speaking, material "noise" is really a spurious background "signature" that is often called noise only because it interferes with, and potentially conceals, the flaw signals to be detected. The main sources of this adverse background signature in thermoelectric NDE are macrostructural features such as case hardening, cold work, texture induced anisotropy, residual stress, etc., while the inherent microstructural inhomogeneity caused by, e.g., the grain structure in polycrystalline metals, is less important because of the lack of sufficient spatial resolution.

The main goal of our research was to lay down the groundwork necessary to develop this new field of nondestructive testing and materials characterization based on noncontacting magnetic detection of thermoelectric currents. In order to achieve this ambitious goal we had to study not only the flaw signals produced by different types of material imperfections, but also the thermoelectric noise or background signature produced by inherent material variations, that together determine the probability of detection (POD) of a certain type of imperfection and 
thereby the ultimate sensitivity threshold of the method. This project involved closely related theoretical and experimental efforts that led to a better understanding of the underlying physical phenomena, the development of new, predictive analytical models, more sensitive experimental procedures, and, ultimately, increased POD for small inclusions and weak material imperfections.

The detectability of a given material flaw by nondestructive means is generally determined by the resulting signal-to-noise ratio $(\mathrm{S} / \mathrm{N})$, where the dominant noise is temporally coherent material noise rather than temporally incoherent electrical noise from the sensor, i.e., from the magnetometer in our case. In order to establish the scientific foundations of the new thermoelectric inspection methods, we first studied a variety of factors that affect the strength of the signals to be detected on one side and the material noise to be rejected on the other side. Needless to say that the same magnetic signature that acts as material noise and limits flaw detectability in one application might very well act as the sought signal in another type of materials characterization. We developed numerous theoretical models capable of predicting the magnetic field produced by thermoelectric currents around spherical, ellipsoidal, and cylindrical inclusions under different types of external thermal excitation as well as the background material signatures produced by weak thermoelectric inhomogeneity and anisotropic texture. These models are based on the well-known coupled transfer equations of thermoelectricity [17]. In all cases, weak thermoelectric coupling was assumed, which was shown to be a very good approximation in all cases of practical importance. The main predictions of these analytical models have been experimentally verified by measurements on well-controlled specimens. Our main theoretical and experimental results are reviewed in the following sections. For brevity, only the basic ideas and main results are presented.

\section{Modeling of the signal from isotropic spherical inclusions}

First, we considered an infinite homogeneous medium containing a spherical inclusion of a different material when the system is subjected to a uniform temperature gradient. After deriving the governing equations for the coupled thermal and electrical fields and satisfying all boundary conditions, first the electrical current densities in the host and the inclusion can be calculated, 
then the magnetic field of the thermoelectric current can be determined by integration using the Biot-Savart law.

Thermoelectricity is a result of intrinsically coupled transport of electricity and heat in metals. The electrical current density $j$ and thermal flux $h$ produced by a given combination of electrochemical potential $\Phi$ and temperature $T$ distributions are given by [17]

$$
\left[\begin{array}{l}
j \\
h
\end{array}\right]=\left[\begin{array}{ll}
\sigma & \varepsilon \\
\bar{\varepsilon} & \kappa
\end{array}\right]\left[\begin{array}{l}
-\nabla \Phi \\
-\nabla T
\end{array}\right],
$$

where $\sigma$ denotes the electrical conductivity measured at uniform temperature, $\kappa$ is the thermal conductivity for zero electrical field, and $\varepsilon$ and $\bar{\varepsilon}$ are thermoelectric coupling coefficients that can be expressed by the absolute thermoelectric power $S$ of the material as $\varepsilon=\sigma S$ and $\bar{\varepsilon}=\sigma S T$. The thermal conductivity for zero electrical field $\kappa$ can be easily expressed by the thermal conductivity of the material for zero electrical current $k$, which is often easier to determine experimentally, as $\kappa=k+\sigma S^{2} T$. The difference between these two thermal conductivities is due to the thermoelectric coupling in the material. We can write that $\kappa=k(1+\eta)$, where $\eta=\sigma S^{2} T / k$ is a dimensionless factor that provides a measure of the degree of coupling between thermal and electrical transports. For typical metals, the coupling factor is relatively small somewhere between $10^{-3}$ and $10^{-2}$, an important fact that will be exploited in our following calculations.

The total energy flux $h+\Phi j$ includes the thermal flux plus an additional term representing the changing electrochemical potential of the electrons. The rate at which heat is evolved, per unit volume, at any point in the material is

$$
\nabla \cdot h+\nabla \Phi \cdot j=-C \dot{T}
$$

where $C$ is the specific heat and we exploited Maxwell's law that $\nabla \cdot j=0$. If we now assume the coupling coefficients $\varepsilon$ and $\bar{\varepsilon}$ to be small such that their individual squares and products can be neglected, then for thermal loading we can neglect the nonlinear term, namely $\nabla \Phi \cdot j$, in Eq. (2). Finally, for steady state thermal loading, this equation further reduces to 
$\nabla \cdot h=0$. Imposing these requirements on Eq.(1) and noting that $\sigma \kappa-\varepsilon \bar{\varepsilon} \neq 0$ dictates that the Laplacian of $T$ and $\Phi$ vanish individually, namely

$$
\nabla^{2} T=0 \text { and } \nabla^{2} \Phi=0
$$

For a homogeneous isotropic medium, $\sigma, \kappa, \varepsilon$ and $\bar{\varepsilon}$ are scalar quantities that do not depend on the spatial coordinates, though generally they do depend on temperature, especially $\varepsilon$ and $\bar{\varepsilon}$. In the first-order approximation of Eq. (1), the temperature dependence of $\sigma$ can be neglected and the curl of the thermoelectric current $\nabla \times j=-\sigma \nabla \times \nabla \Phi-\varepsilon \nabla \times \nabla T$ $-(\partial \varepsilon / \partial T) \nabla T \times \nabla T$ is zero. Actually, this outcome does not change even if the temperature dependence of $\sigma$ is accounted for by an additional term $-(\partial \sigma / \partial T) \nabla T \times \nabla \Phi$, as $\nabla T$ will be parallel with $\nabla \Phi$. Since the divergence of the thermoelectric current is inherently zero, in the absence of an external electric source, the current density itself must be identically zero everywhere in the medium. This means that, regardless of the size, shape, and material properties of a homogeneous isotropic specimen, no thermoelectric currents will be generated by any type of heating or cooling. In other words, the presence of any magnetically or otherwise detected thermoelectric current will positively identify the specimen as either inhomogeneous or anisotropic.

\section{II.1 Infinite homogeneous medium containing a spherical inclusion}

First, we considered an infinite homogeneous medium (host) containing a spherical inclusion of a different material having radius $a$. To differentiate between the properties of the two media, we designate those of the inclusion by a prime. The system is subjected to a thermal flux $h_{0}$ far away from the inclusion and directed along the $x_{3}$-axis of the Cartesian coordinate system $\left(x_{1}, x_{2}, x_{3}\right)$ as illustrated in Figure 2. 


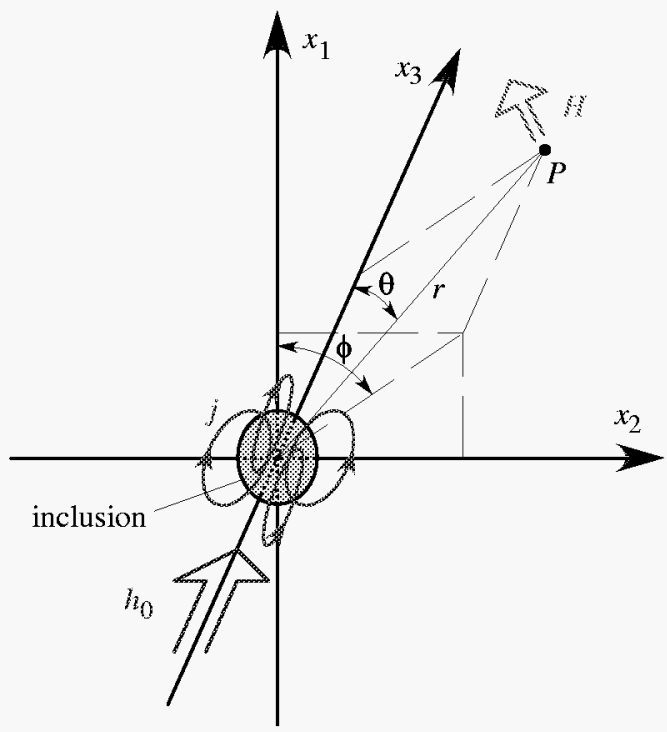

Figure 2 The coordinate system used to study the magnetic field produced by the thermoelectric currents around a spherical inclusion embedded in an otherwise homogeneous specimen.

In the absence of the inclusion, solutions to the above presented coupled field equations are

$$
T=T_{0}=-\frac{h_{0}}{\kappa} x_{3} \text { and } \Phi=\Phi_{0}=\frac{\varepsilon h_{0}}{\kappa \sigma} x_{3}
$$

It is advantageous to introduce a spherical polar coordinate system $(r, \theta, \varphi)$ so that $\theta$ is the polar angle measured from the $x_{3}$ direction and $\varphi$ is the azimuthal angle measured from the $x_{1}$ direction. In these spherical coordinates, Eq. (4) can be expressed as

$$
T_{0}=-\frac{h_{0}}{\kappa} r \cos \theta \text { and } \Phi_{0}=\frac{\varepsilon h_{0}}{\kappa \sigma} r \cos \theta
$$

With these solutions as a guide, in the presence of the spherical inclusion we seek solutions of the form

$$
T=f(r) \cos \theta \text { and } \Phi=g(r) \cos \theta
$$


These solutions have to satisfy Eq. (3). For the axisymmetric situation under consideration, the Laplacian operator in spherical coordinates is widely available and is given as

$$
\nabla^{2}=\frac{\partial^{2}}{\partial r^{2}}+\frac{2}{r} \frac{\partial}{\partial r}+\frac{1}{r^{2}}\left(\frac{\partial^{2}}{\partial \theta^{2}}+\cot \theta \frac{\partial}{\partial \theta}\right)
$$

Combinations of Eqs. (6) and (7) lead to the following formal solutions for the temperature and electric potential for both the host and the inclusion

$$
T=\left(A r+\frac{B}{r^{2}}\right) \cos \theta, \Phi=\left(A^{*} r+\frac{B^{*}}{r^{2}}\right) \cos \theta, T^{\prime}=D r \cos \theta, \Phi^{\prime}=D^{*} r \cos \theta
$$

where bondedness of the solutions at $r=0$, i.e., at the center of the inclusion, is satisfied and $A, A^{*}, B, B^{*}, D$, and $D^{*}$ are currently unknown constants to be determined from the appropriate boundary conditions. At the interface between the host and the inclusion $(r=a)$, the interface continuity conditions require that both the temperature and the electrical potential be continuous, namely $T=T^{\prime}, \Phi=\Phi^{\prime}$. Furthermore, the normal (radial) components of both the electrical current density and thermal flux are also continuous at $r=a, j_{r}=j_{r}{ }_{r}$ and $h_{r}=h_{r}^{\prime}$.

It should be mentioned that the continuity of the thermal flux is an approximation based on $\nabla \cdot h=0$., which is used instead of the continuity of the total energy flux of Eq. (2) in the weak thermoelectric coupling approximation. As such, it clearly neglects the so-called Peltier heat generated at the interface between the host and the inclusion as a result of the weak thermoelectric currents. The unknown constants $A, A^{*}, B, B^{*}, D$, and $D^{*}$ can be determined by imposing the above boundary conditions on the formal solutions Eq. (8) and requiring that in the limit of $r \rightarrow \infty$ the solutions reduce to Eq. (5). After some algebraic reductions while exploiting small coupling we obtain [10] for $j$ and $j^{\prime}$

$$
j=\frac{a^{3} h_{0}}{r^{3}} G_{s}\left(2 \cos \theta e_{r}+\sin \theta e_{\theta}\right) \text { and } j^{\prime}=h_{0} G_{s}\left(2 \cos \theta e_{r}-2 \sin \theta e_{\theta}\right)
$$

where 


$$
G_{s}=3 \frac{\varepsilon^{\prime} \sigma-\varepsilon \sigma^{\prime}}{\left(\sigma^{\prime}+2 \sigma\right)\left(\kappa^{\prime}+2 \kappa\right)} .
$$

Of course similar expressions can be obtained for $h$ and $h^{\prime}$, but need not be reported here. It now remains to obtain expressions for the magnetic field components $H_{\varphi}$ and $H_{\varphi}^{\prime}$. We recall that the electrical current density $j$ is related to the magnetic field $H$ by $\nabla \times H=j$. In spherical coordinates, we have

$$
\nabla \times H=\frac{1}{r}\left(\frac{\partial H_{\varphi}}{\partial \theta}+\cot \theta H_{\varphi}\right) e_{r}-\left(\frac{\partial H_{\varphi}}{\partial r}+\frac{H_{\varphi}}{r}\right) e_{\theta}
$$

where we exploited the fact that, due to the axial symmetry of the problem, both $H_{r}$ and $H_{\theta}$ identically vanish everywhere. From Eqs. (11) then

$$
H_{\varphi}=a^{3} h_{0} G_{s} \frac{\sin \theta}{r^{2}} \text { and } H_{\varphi}^{\prime}=h_{0} G_{s} r \sin \theta
$$

\section{II.2 Infinite homogeneous medium containing a cylindrical inclusion}

For an infinitely extended cylindrical inclusion along the $x_{1}$-direction, the field distributions will be independent of $x_{1}$. Using cylindrical coordinates, formal solutions will lead to a set of equations similar to those of Eq. (8) with the minor modification of replacing the $1 / r^{2}$ coefficients of $B$ and $B^{*}$ with $1 / r$ and assuming that $A, A^{*}, B, B^{*}, D$, and $D^{*}$ are new constants that need to be derived from the continuity and boundary conditions once again. Following the steps used in deriving these constants for the spherical inclusion, we get [10]

$$
j=\frac{a^{2} h_{0}}{r^{2}} G_{c}\left(\cos \theta e_{r}+\sin \theta e_{\theta}\right) \text { and } j^{\prime}=h_{0} G_{C}\left(\cos \theta e_{r}-\sin \theta e_{\theta}\right) \text {, }
$$

where $G_{c}$ now takes the appropriate form of

$$
G_{c}=2 \frac{\varepsilon^{\prime} \sigma-\varepsilon \sigma^{\prime}}{\left(\sigma^{\prime}+\sigma\right)\left(\kappa^{\prime}+\kappa\right)}
$$


It is important to recognize that, except for some numerical constants, the thermoelectric contrast represented by $G_{c}$ is essentially the same as the previously derived $G_{s}$ for spherical inclusions. After similar analysis to the one for spherical inclusions, these current distributions lead to a magnetic field parallel to the axis of the cylindrical inclusion as follows

$$
H_{1}=a^{2} h_{0} G_{c} \frac{\sin \theta}{r} \text { and } H_{1}^{\prime}=h_{0} G_{c} r \sin \theta
$$

\section{II.3 Numerical results}

In the previous sections, we assumed that the host is infinite so that the analysis could be simplified as a result of the perfect axial symmetry. In particular, due to this axial symmetry, $T, \Phi, h, j$, and $H$ were all independent of $\varphi$, and $h_{\varphi}, j_{\varphi}, H_{r}$, and $H_{\theta}$ all vanished. Needless to say that this assumption renders the problem somewhat hypothetical since the magnetic field cannot be practically measured inside the specimen. In order to realistically model a large but finite-size specimen, we have to either truncate or section the infinite specimen so that the measurement of the magnetic field could be done on the outside. Generally, this requirement renders the calculations very difficult since the spatial distribution of the thermoelectric current density becomes distorted as all the current that would otherwise spread out as $1 / r^{3}$ is squeezed inside the contour of the finite specimen. In order to show how dramatically this will affect the magnetic field, let us consider a cylindrical specimen of radius $b$ with its axis along the $x_{3}$ direction. Unless $b>>a$, the thermoelectric current density distribution will be significantly different from that in an infinite medium, but it will be still axisymmetric. In this case, the resulting magnetic field can be readily calculated from Stokes' theorem

$$
\oint H d s=\iint j d A,
$$

where the integrations on the left and right sides are carried out over the circumference and the area of the same continuous surface, respectively. Outside the specimen $(r>b), j=0$ so that the total electrical current on the right side of Eq. (16) is identically zero, therefore the magnetic 
field completely vanishes. It is clear that, by virtue of Stokes' theorem, any other axisymmetric arrangement would also result in the complete disappearance of the external magnetic field. In other words, the magnetic field to be measured in the case of an embedded inclusion is caused by asymmetric distortion of the thermoelectric current distribution and it will be inevitably rather weak. This means that noncontacting magnetic detection of a spherical inclusion is possible only if it is close enough to the surface of the specimen so that the thermoelectric currents are deflected by the surface contour. Otherwise, the thermoelectric currents form a toroid that produces no magnetic field on the outside.

To simplify the following calculations, all spatial coordinates will be normalized to the radius of the spherical inclusion as $\boldsymbol{\xi}=x / a$. The magnetic field can be also written in a normalized form as $H=H_{0} F(\boldsymbol{\xi})$, where $H_{0}=a h_{0} G_{s}$ combines the size of the inclusion $a$, the thermoelectric contrast $G_{s}$ and the externally induced heat flux $h_{0}$ into a single scalar constant characterizing the strength of the magnetic field while $F(\boldsymbol{\xi})$ is a universal spatial distribution function for all spherical inclusions. In order to better facilitate the estimation of the absolute strength of the magnetic field in a given experimental arrangement, we can re-write $H_{0}$ with the temperature gradient $\nabla T$ that would prevail in the vicinity of the inclusion if it were not there. From Eq. (4), $h_{0}=-\kappa \nabla T$ and

$$
H_{0}=-a \nabla T \sigma S_{S R} \Gamma_{s},
$$

where $S_{S R}=S^{\prime}-S$ is the relative thermoelectric power of the inclusion with respect to the host and $\Gamma_{s}$ is the normalized contrast coefficient

$$
\Gamma_{s}=\frac{3}{\left(1+2 \frac{\sigma}{\sigma^{\prime}}\right)\left(2+\frac{\kappa^{\prime}}{\kappa}\right)} .
$$

For a cylinder, the corresponding normalized contrast coefficient is

$$
\Gamma_{c}=\frac{2}{\left(1+\frac{\sigma}{\sigma^{\prime}}\right)\left(1+\frac{\kappa^{\prime}}{\kappa}\right)} .
$$


It should be noted that, for weak material inhomogeneity $\left(\kappa^{\prime} \approx \kappa\right.$ and $\left.\sigma^{\prime} \approx \sigma\right)$, the normalized contrast coefficients of spherical and cylindrical inclusions approach $1 / 3$ and $1 / 2$, respectively.

\section{II.4 Half-space with a surface-breaking spherical inclusion}

The closer the inclusion to the surface, the stronger the outside magnetic field. Maximum detectability is reached when the inclusion is cut halfway through by the surface as it is shown in Figure 3. In this case, the coupled thermoelectric problem in the remaining half-space is exactly the same as it was before, though the resulting magnetic field is not axisymmetric anymore. In particular, $T, \Phi, h$ and $j$ will be still independent of $\varphi$ and $h_{\varphi}$ and $j_{\varphi}$ both vanish in both the host and the inclusion, but $H$ will now depend on $\varphi$, and neither $H_{r}$ nor $H_{\theta}$ will automatically vanish. Actually, it is advantageous to describe the magnetic field in Cartesian coordinates since in that way at least one of the components, namely $H_{3}$, does identically vanish. This can be easily illustrated by recasting Eq. (12) as follows

$$
F=\frac{-\xi_{2} i+\xi_{1} j}{\left(\xi_{1}^{2}+\xi_{2}^{2}+\xi_{3}^{2}\right)^{3 / 2}}
$$

for the host and

$$
F=-\xi_{2} i+\xi_{1} j
$$

for the inclusion, where $i$ and $j$ are the Cartesian unit vectors in the $x_{1}$ and $x_{2}$ directions, respectively.

Since the magnetic field of the thermoelectric current is no longer axisymmetric, the sought magnetic field cannot be simply calculated from Stokes' theorem and we have to rely on the Biot-Savart law

$$
H(x)=\iiint_{V} \frac{j \times(x-X)}{4 \pi|x-X|^{3}} d X_{1} d X_{2} d X_{3},
$$

where the integration is carried out over the entire half-space, $V$, and inside the inclusion $j$ should be substituted by $j^{\prime}$. 


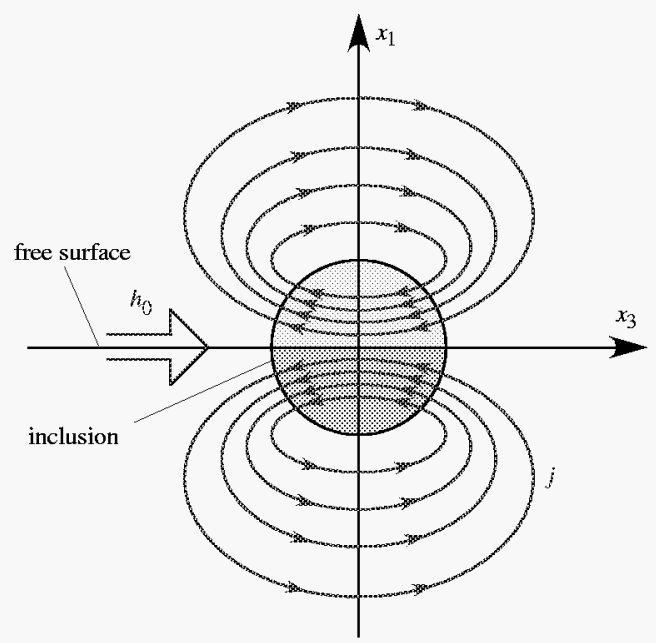

Figure 3 A schematic diagram of a surface-breaking spherical inclusion and the resulting symmetric thermoelectric current distribution.

\section{II.5 Half-Space with a subsurface spherical inclusion}

In order to simulate fully embedded hidden inclusions, we will assume that the center of a spherical inclusion lies below the surface at a depth $d$ deeper than its radius, i.e., it is not breaking the surface at all. In this case, symmetry to the sectioning plane can be retained by considering a pair of spherical inclusions as shown in Figure 4. By assuming that the inclusions are only slightly different from the host or the separation $2 d$ between them is large with respect to their radius $a$, the interaction between the two inclusions can be neglected and the resulting thermoelectric current distribution can be approximated simply by superimposing the currents produced by the individual inclusions.

Because of its simplicity, we can adapt this approximation to spherical inclusions embedded below the surface at shallow depths only when the thermal and electrical conductivities of the inclusion are similar to those of the host, i.e., in the weak contrast limit. In this case, we have to replace $\xi_{1}$ by $\xi_{1}+\delta$ in Eqs. (20) and (1), where $\delta=d / a$ denotes the normalized depth, so that

$$
F=\frac{-\xi_{2} i+\left(\xi_{1}+\delta\right) j}{\left[\left(\xi_{1}+\delta\right)_{1}^{2}+\xi_{2}^{2}+\xi_{3}^{2}\right]^{3 / 2}}
$$

for the host and 


$$
F=-\xi_{2} i+\left(\xi_{1}+\delta\right) j
$$

for the inclusion. Directly on the surface $\left(\xi_{1}=0\right)$, the normal and tangential components of the magnetic field can be readily obtained from Eq. (23) as follows

$$
F_{1}=-\frac{\xi_{2}}{\left(\delta^{2}+\xi_{2}^{2}+\xi_{3}^{2}\right)^{3 / 2}} \text { and } F_{2}=\frac{\delta}{\left(\delta^{2}+\xi_{2}^{2}+\xi_{3}^{2}\right)^{3 / 2}}
$$

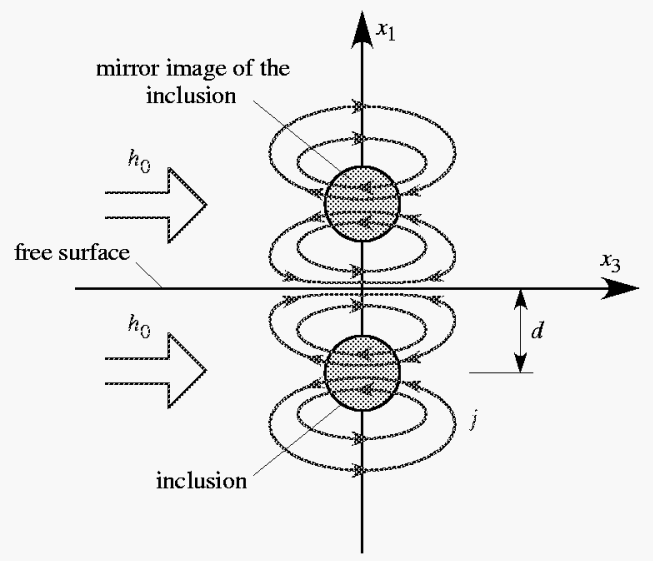

Figure 4 A schematic diagram of the inclusion buried below the surface and its mirror image above it.

Figure 5 shows the surface scan $\left(\xi_{1}=0, \xi_{2}, \xi_{3}\right)$ of the normal $\left(F_{1}\right)$ component of the normalized magnetic field for a subsurface spherical inclusion at a normalized depth $\delta=1$ based on Eq. (25). As it could be expected qualitatively from Figure 1, the thermoelectric currents flow in opposite directions along two loops on the opposite sides of the inclusion relative to the direction of the heat flux, therefore the magnetic field is asymmetric with respect to this direction. One of the most crucial questions to be answered is how deeply inclusions can be buried and still detected. Figure 6 shows the maximum normal $F_{1}\left(\xi_{1}=0, \xi_{2}=-\delta / \sqrt{2}, \xi_{3}=0\right)$ and tangential $F_{2}\left(\xi_{1}=0, \xi_{2}=0, \xi_{3}=0\right)$ components of the normalized magnetic field on the surface for a subsurface spherical inclusion as a function of the normalized depth $\delta$. The magnetic field to be detected rapidly decreases with the depth of the inclusion. The normal component drops from $F_{1} \approx 0.4$ at $\delta=1$ to as low as $F_{1} \approx 0.024$ at $\delta=3$. This sharp decay 
bodes ill for detection of inclusions buried deeply in the specimen, although with high-sensitivity magnetometers we might tolerate $F_{1}$ values as low as $10^{-3} \div 10^{-4}$.

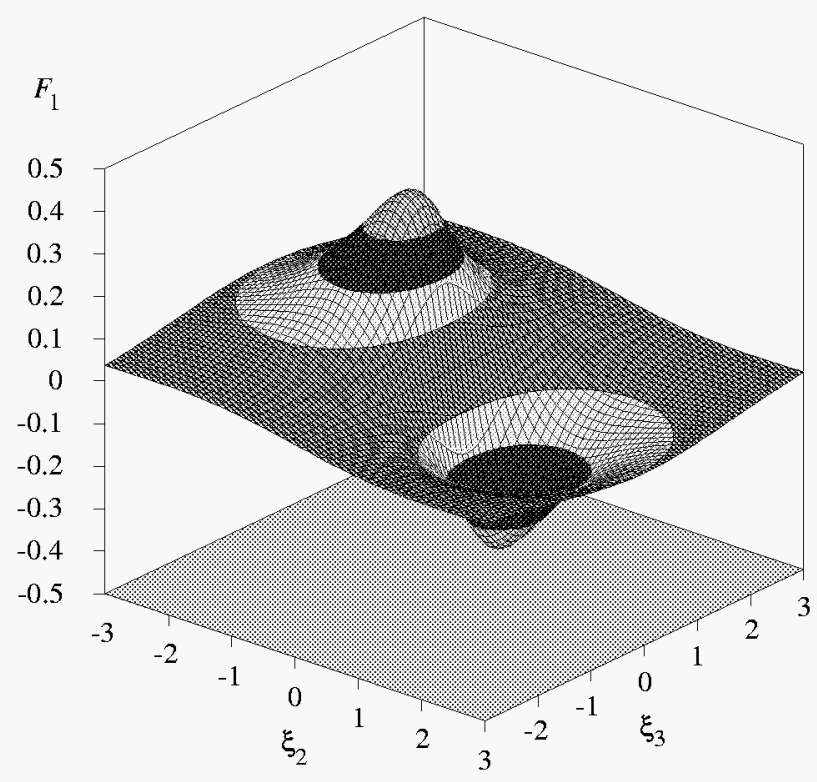

Figure 5 Surface scan $\left(\xi_{1}=0, \xi_{2}, \xi_{3}\right)$ of the normal $\left(F_{1}\right)$ component of the normalized magnetic field for a subsurface spherical inclusion at a depth $d=a$.

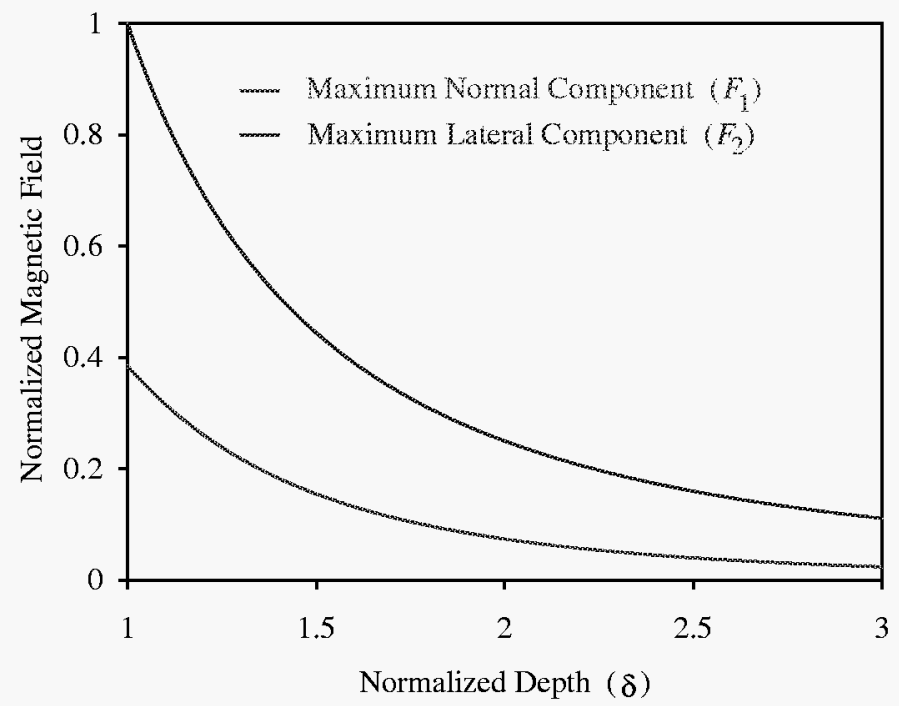

Figure 6 The maximum normal $F_{1}\left(\xi_{1}=0, \xi_{2}=-\delta / \sqrt{2}, \xi_{3}=0\right)$ and tangential $F_{2}\left(\xi_{1}=0, \xi_{2}=0, \xi_{3}=0\right)$ components of the normalized magnetic field on the surface for a subsurface spherical inclusion as a function of the normalized depth. 
III. Experimental investigation of the signal from isotropic spherical inclusions

One of the primary goals of our research project was to experimentally verify that our theoretical models can accurately predict the thermoelectric signature of surface-breaking, subsurface, and deeply embedded inclusions. Our experimental results on spherical tin inclusions in a copper host provided excellent verification of our analytical predictions and also highlighted the crucial areas where significant future improvements are needed. In this section, we will briefly discuss the experimental setup and procedure used to verify that the above described analytical model truthfully captures the main features of the thermoelectrically generated magnetic field and accurately predicts its magnitude over a wide range of inclusion sizes and lift-off distances.

III.1 Predictions for a surface-breaking inclusion

Figure 7 shows the two-dimensional distributions of the normal component of the magnetic field $F_{1}\left(\xi_{2}, \xi_{3}\right)$ taken in planes parallel to the free surface at four different lift-off distances $\left(\xi_{1}=0\right.$, $0.5,1$, and 2) for a semi-spherical inclusion with its center lying on the surface. The characteristic shape produced by the positive and negative peaks in the magnetic field distribution are very typical in noncontacting thermoelectric detection of inclusions and can be easily exploited by digital image processing and feature extraction techniques to increase the probability of detection for inclusions. The larger the lift-off distance, the lower the peak magnetic field and the wider the field distribution. The latter can be quantitatively characterized by the lateral distance of the peaks from the center of the inclusion, $w$, which we are going to call the half-width of the bi-polar signature. Since the field distribution remains very similar regardless of the lift-off distance, it is reasonable to choose these two parameters, namely the peak magnetic field and the half-distance between the peaks, to characterize the whole field distribution. Figure 8 shows these two parameters as functions of the normalized lift-off distance. The solid lines are numerical results while the dashed lines are far-field asymptotes. At small lift-off distances, the peaks are located directly above the circumference of the inclusion, i.e., $w \approx 1$, and their magnitude approaches 0.5 [10]. At large distances, the field distribution spreads out so that the peaks occur at $\phi \approx 45^{\circ}$, i.e., $w \approx \xi_{1} / \sqrt{2}$, and their magnitude is inversely proportional to the square of the normalized lift-off distance. 
a) $\xi_{1}=0$

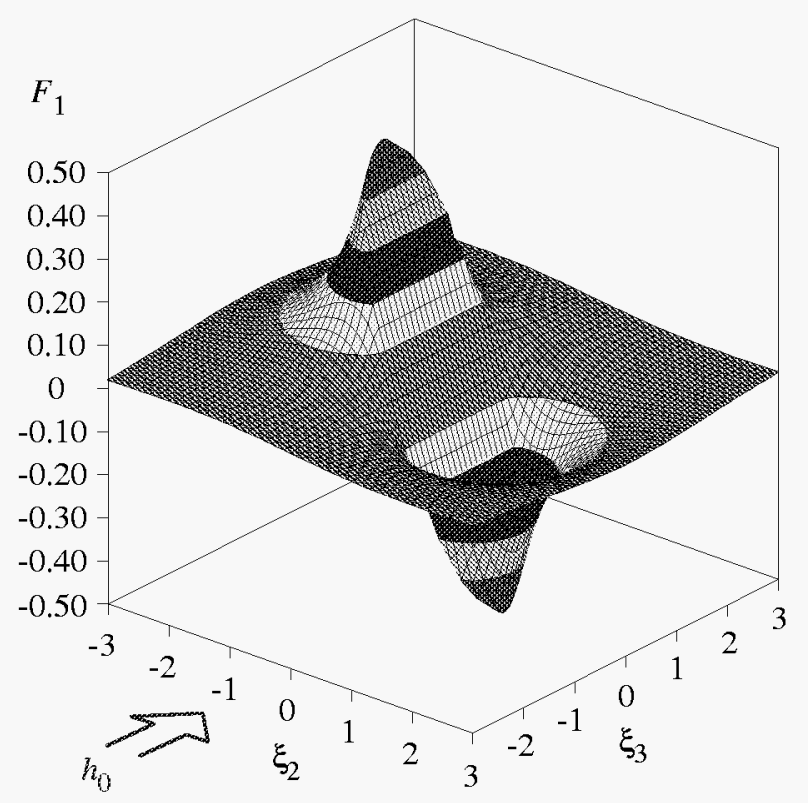

c) $\xi_{1}=1$



b) $\xi_{1}=0.5$

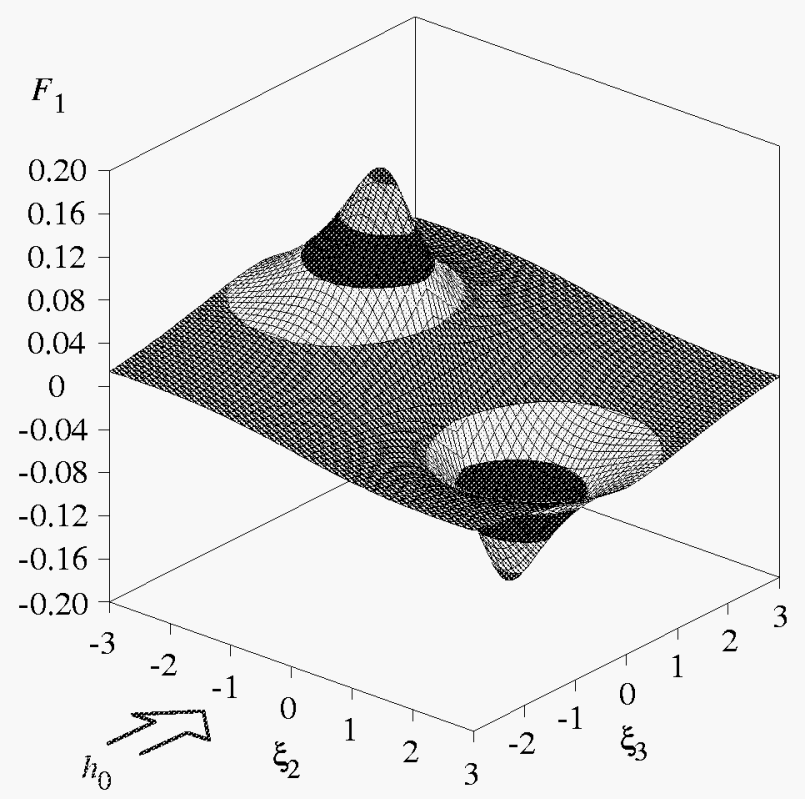

d) $\xi_{1}=2$

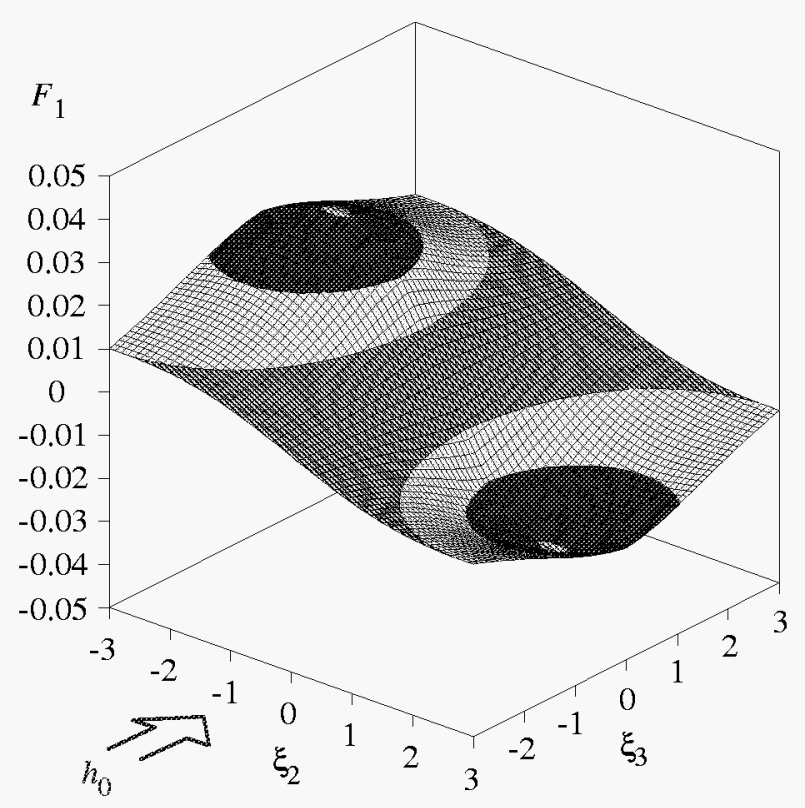

Figure 7 Two-dimensional distributions of the normal component of the magnetic field taken in four different planes parallel to the free surface at different lift-off distances for a semi-spherical inclusion with its center lying on the surface. 


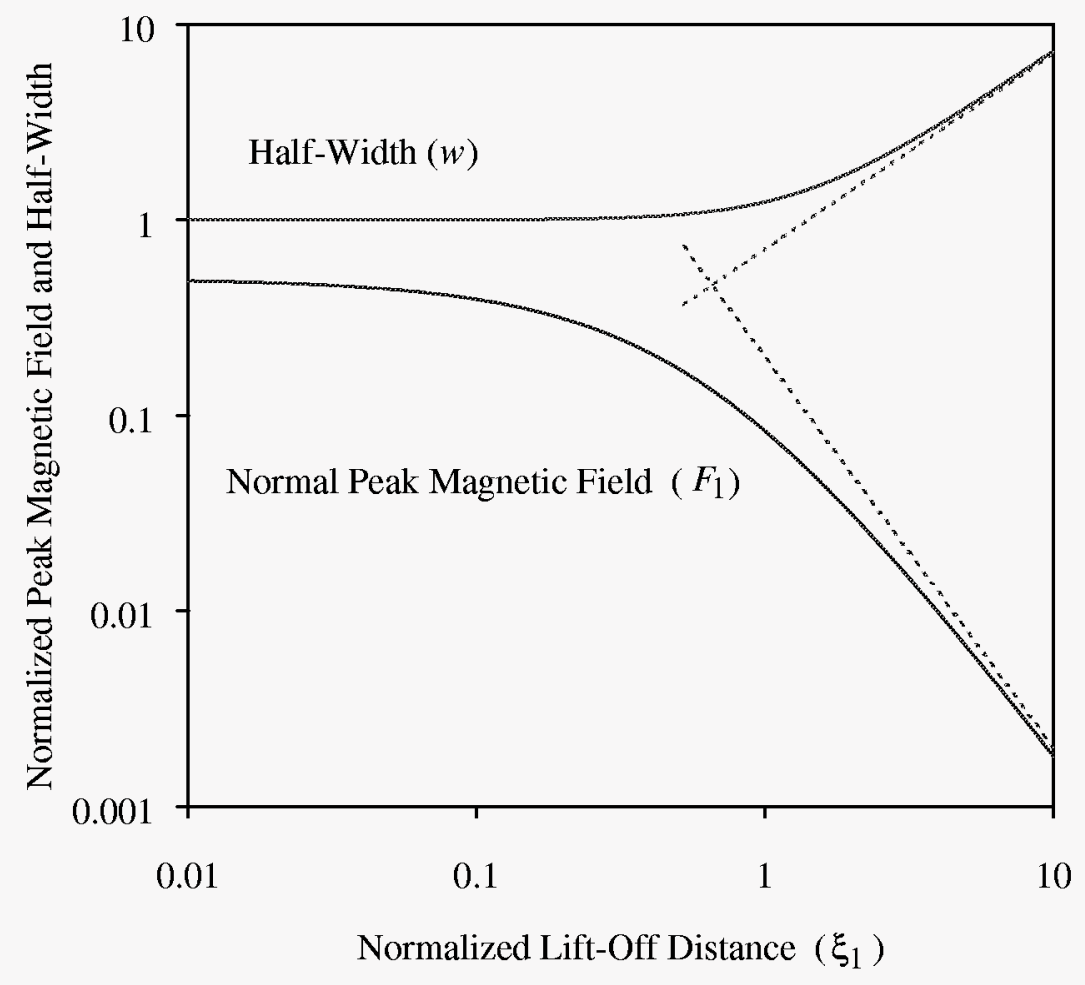

Figure 8 The two principal parameters of the field distribution, namely the peak of the normalized magnetic field and the half-distance between the peaks, as functions of the normalized lift-off distance. The solid lines are numerical predictions while the dashed lines are far-field asymptotes.

\section{III.2 Experimental method}

Figure 9 shows a schematic diagram of the experimental arrangement. We prepared a series of semi-spherical pure tin inclusions embedded in two pure copper bars of $12.7 \mathrm{~mm} \times 38.1 \mathrm{~mm} \times$ $500 \mathrm{~mm}$ dimensions. First, we made semi-spherical holes by milling, then we heated the specimens to approximately $300{ }^{\circ} \mathrm{C}$ and filled the holes with molten tin, and finally milled the surface flat after the specimen has cooled down. The diameter of the inclusions varied from 2.38 $\mathrm{mm}$ to $12.7 \mathrm{~mm}$ and the center of each inclusion was at the level of the specimen's surface. The distance between inclusions was approximately $75 \mathrm{~mm}$ to avoid interference between their individual magnetic fields. Both ends of the cooper bar were perforated by a series of holes and 
equipped with sealed heat exchangers to facilitate efficient heating and cooling and then mounted on a non-magnetic translation table for scanning.

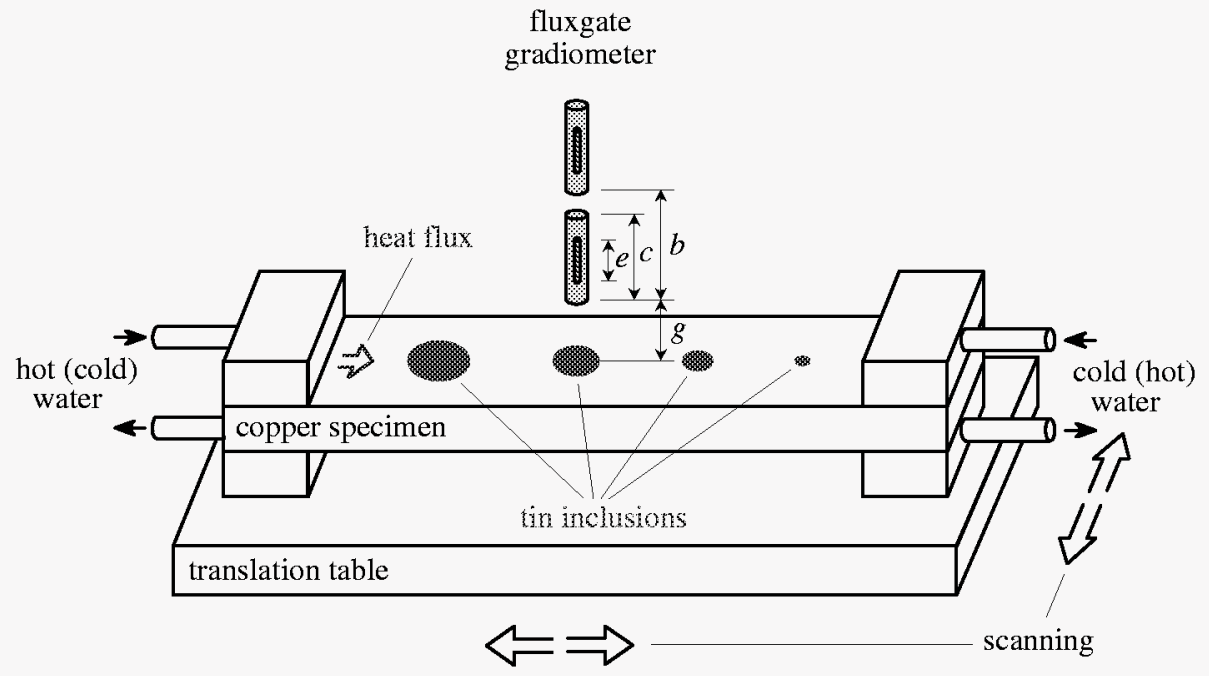

Figure 9 A schematic diagram of the experimental arrangement.

The relevant physical properties of pure copper and tin were taken from standard references with the exception of the absolute thermoelectric power of tin at room temperature, which was measured by a Koslow TE-3000 thermoelectric instrument on the largest inclusion itself since it was found to be significantly affected by melting and subsequent recrystallization during sample preparation [11]. The TE-3000 is a conventional thermoelectric alloy tester used in NDT, that provides relative readings only, therefore it was first absolute calibrated by materials of known absolute thermoelectric power such as standard thermocouple alloys like Alumel and Chromel and pure copper.

In order to better separate the sought magnetic signals of truly thermoelectric origin from potentially much stronger spurious artifacts, we had to adopt a series of protective measures. Since the earth's static magnetic field is about $100 \mu \mathrm{T}$, i.e., orders of magnitude stronger than the fields generated by thermoelectric currents around metallic inclusions, dc measurements are rendered completely useless. In our experiments, we used ac coupling with a high-pass filter of very low cut-off frequency at $0.01 \mathrm{~Hz}$. Since sufficiently fast alternating heating could not be implemented because of the inherently sluggish thermal response of the specimen, all measurements were done under steady-state thermal condition that was achieved in a few 
minutes after starting the heating and cooling. The pseudo-dynamic magnetic signals required for ac detection were produced by laterally (normal to the heat flux) scanning the specimen at a speed of $20 \mathrm{~mm} / \mathrm{s}$. As we have previously mentioned, the magnetic field is asymmetric to the principal direction of heating, therefore the signal to be detected does not exhibit a significant de component. In addition to the relatively fast lateral scanning ("line" direction), we also scanned the specimens at a much lower rate in the axial direction ("frame" direction). In this way, a 76.2 $\mathrm{mm} \times 76.2 \mathrm{~mm}$ scan of $200 \times 200$ pixels took about 13 minutes. It should be mentioned that it is absolutely necessary to move the specimen with respect to the magnetometer rather than the other way around. As a result of the distortions caused by the presence of ferromagnetic objects in the surrounding, the earth's magnetic field is inhomogeneous enough that any motion of the magnetometer would produce unacceptable levels of spurious signals. Of course all the moving parts of the translation table have to be made of nonmagnetic materials and even nonmagnetic materials of high susceptibility should be avoided.

In order to reach the high sensitivity required in these measurements, non-stationary extraneous magnetic fields also have to be rejected. Most of the ac magnetic interference occurs at the $60 \mathrm{~Hz}$ mains frequency and its upper harmonics. These signals were effectively eliminated by a very sharp (6-pole) low-pass filter of $20 \mathrm{~Hz}$ cut-off frequency. These methods based on the frequency spectra of the spurious magnetic signals cannot be applied to reduce extraneous signals in the very frequency range were the thermoelectric signals are expected, namely between $0.01 \mathrm{~Hz}$ and $20 \mathrm{~Hz}$. In this range, we can separate the sought magnetic signals from unwanted background signals based on their spatial rather than temporal dependence. The signals to be detected are generated in the close vicinity of the magnetometer therefore they significantly vary from point to point, i.e., they exhibit strong gradients. Extraneous signals typically originate at larger distances from the magnetometer, therefore they smoothly vary from point to point, i.e., they exhibit relatively small gradients. In order to exploit this difference, we used a pair of detectors in a gradiometric arrangement. The primary sensor closer to the specimen picks up a much stronger signal from the inclusion than the secondary sensor further away, while the two sensors exhibit essentially the same sensitivity for sources at large distances. The baseline distance ( $b$ in Figure 9) was chosen to be $32 \mathrm{~mm}$ in our case. Further reduction of the baseline distance would improve the rejection of extraneous signals but would also reduce the sensitivity to the thermoelectric signals to be detected. 
All these measures taken to eliminate the adverse effects of magnetic interference from the surroundings cannot assure that the specimen itself does not produce a spurious magnetic signature caused by ferromagnetic contamination or high susceptibility of the material itself. In order to positively identify magnetic signals of purely thermoelectric origin, we always recorded a "baseline" scan without external heating and cooling. In addition, the experimental data were always obtained as the difference between measurements taken at opposite heating directions. As it is indicated in Figure 9, alternating the heating and cooling directions was achieved simply by changing the hot and cold water supplies connected to the heat exchangers and waiting a few minutes until steady-state conditions were reached.

The ends of the copper bar were simultaneously heated and cooled by running water to temperatures of $\approx+10{ }^{\circ} \mathrm{C}$ and $\approx+40{ }^{\circ} \mathrm{C}$, respectively. The actual temperature difference between the ends of the bar was monitored during the measurements by thermocouple thermometers and the temperature gradient was kept at $0.7{ }^{\circ} \mathrm{C} / \mathrm{cm}$, which is more than sufficient to produce detectable magnetic signals in high-conductivity materials like copper and tin. It should be mentioned that most structural metals exhibit much lower electrical conductivity than copper. For example, in Ti-6Al-4V, the most popular aerospace titanium alloy, the electrical conductivity is only $\sigma \approx 5.8 \times 10^{5} \mathrm{~A} / \mathrm{Vm}$, i.e., two orders of magnitude lower than that of copper, therefore the expected magnetic signals are also proportionally lower.

Like most other methods used in NDT, the noncontacting thermoelectric method is ultimately limited by temporally coherent material noise rather than temporally incoherent electrical noise that could be easily eliminated by simple time-averaging. The main sources of such adverse background signals in thermoelectric NDT are macrostructural features such as case hardening, cold work, texture-induced anisotropy, residual stress, etc., while small-scale microstructural features such as grains are less important because of the lack of sufficient spatial resolution. To illustrate the adverse effect of the material-dependent background signature on the accuracy of noncontacting thermoelectric measurements, Figure 10a shows the magnetic pictures of a typical 12.7-mm-thick, 38.1-mm-wide pure copper bar stock for opposite directions of heating. There is a relatively strong baseline signature running parallel to the length of the bar, that does not change when the specimen is flipped upside down but changes sign when the heating direction is reversed. The peak magnetic flux density of this baseline is approximately 8 
$\mathrm{nT}$, which is actually larger than the signals produced by the smallest tin inclusions used in our experiments.

These effects are much smaller in a bar cut from a larger plate, but can be completely eliminated only by appropriate annealing as shown in Figures $10 \mathrm{~b}$ and 10c. The background signature in a similar copper bar machined from a plate is only approximately $2 \mathrm{nT}$, while it is less than $0.5 \mathrm{nT}$ in the annealed ( 30 minutes at $700{ }^{\circ} \mathrm{C}$ in a vacuum furnace) copper bar stock. Since this baseline is essentially the same everywhere along the length of the bar, its adverse effect on flaw detection can be significantly reduced by subtraction. The copper specimens used in our following experiments were all cut from a 12.7 -mm-thick plate, but instead of the rather troublesome annealing process we chose to simply subtract the baseline signature whenever it reached above $10 \%$ of the signal from the inclusion.

a) bar stock, $\approx 8 \mathrm{nT}$

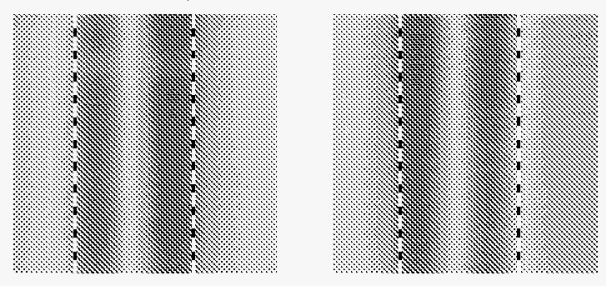

b) from plate, $\approx 2 \mathrm{nT}$

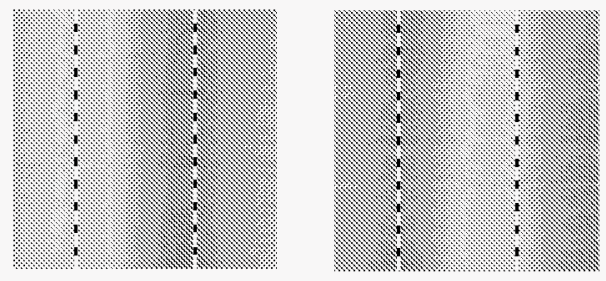

c) bar stock after annealing, $<0.5 \mathrm{nT}$

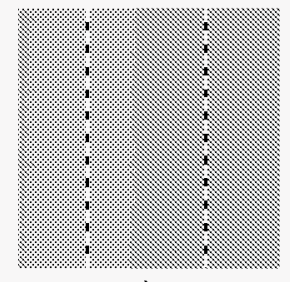

介t

$\nabla T$

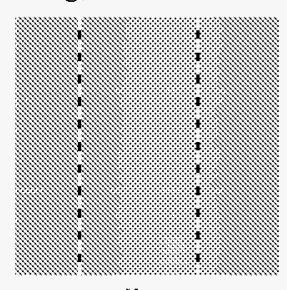

$\forall$

Figure 10 Case hardening induced background signature in copper rods $\left(\nabla T \approx 0.5^{\circ} \mathrm{C} / \mathrm{cm}\right.$, $2 \mathrm{~mm}$ lift-off distance, $76.2 \mathrm{~mm} \times 76.2 \mathrm{~mm}$ scanning dimension). 


\section{III.3 Experimental results}

Figure 11 shows examples of the magnetic images obtained from two surface-breaking semispherical tin inclusions embedded in copper. These pictures were taken at $g=2 \mathrm{~mm}$ distance above the surface (see Figure 9). This apparent lift-off distance is the actual gap between the tip of the magnetometer probe and the specimen. However, the sensing element of the fluxgate is an $e=15 \mathrm{~mm}$ long ferromagnetic rod centered in a $c=25 \mathrm{~mm}$ long case. For simplicity, we can consider the fluxgate as a point sensor buried at a depth of approximately $12.5 \mathrm{~mm}$ below the tip of the case, i.e., the 2-mm apparent lift-off corresponds to a much larger 14.5-mm actual lift-off distance. The measured magnetic field distributions are very similar in shape to the analytical predictions previously shown in Figure 7. As expected, the characteristic bi-polar lobes change sign when the direction of the temperature gradient in the specimen is reversed. These lobes get larger and the magnitude of the magnetic flux decreases when the lift-off distance is increased.

a) 6.35 -mm-diameter inclusion on the top, $B \approx 33 \mathrm{nT}$
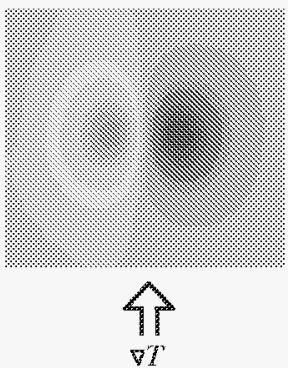

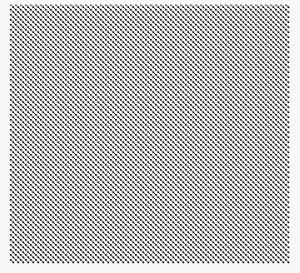

$\nabla T=0$

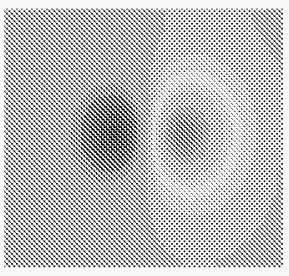

$\frac{15}{\nabla T}$

b) 9.53 -mm-diameter inclusion on the top, $B \approx 104 \mathrm{nT}$

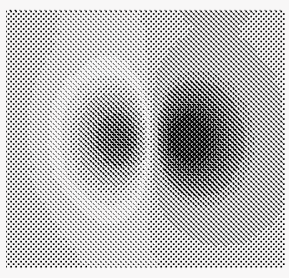

$\underset{\nabla T}{\uparrow}$

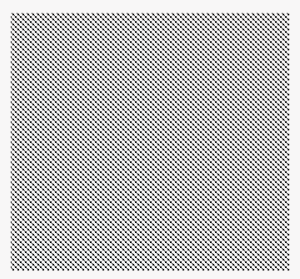

$\nabla T=0$

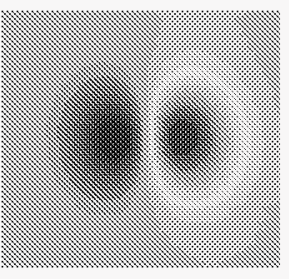

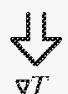

Figure 11 Magnetic images of surface-breaking semi-spherical tin inclusions on the top of a copper host $\left(\nabla T \approx 0.7^{\circ} \mathrm{C} / \mathrm{cm}, 2 \mathrm{~mm}\right.$ lift-off distance, $76.2 \mathrm{~mm} \times 76.2 \mathrm{~mm}$ scanning dimension, the peak magnetic flux density is indicated for comparison). 
Figure 12 shows how the peak magnetic flux density changes with the apparent lift-off distance between 1 and $8 \mathrm{~mm}$ for six inclusions of different diameters between $2.38 \mathrm{~mm}$ and 12.7 $\mathrm{mm}$. The solid lines represent our analytical predictions based on the known material properties [11]. For each inclusion diameter and lift-off distance, the theoretical values were calculated at two sensor positions corresponding to the primary and secondary fluxgates of the gradiometer and the values were subtracted. In most cases, subtraction of the magnetic field measured by the secondary sensor has a negligible effect on the result, but in the case of small inclusions and large lift-off distances the effect can be as high as $15-20 \%$. The lift-off distance was corrected for the depth of the sensing element below the surface of the probe, but no other adjustments were made.

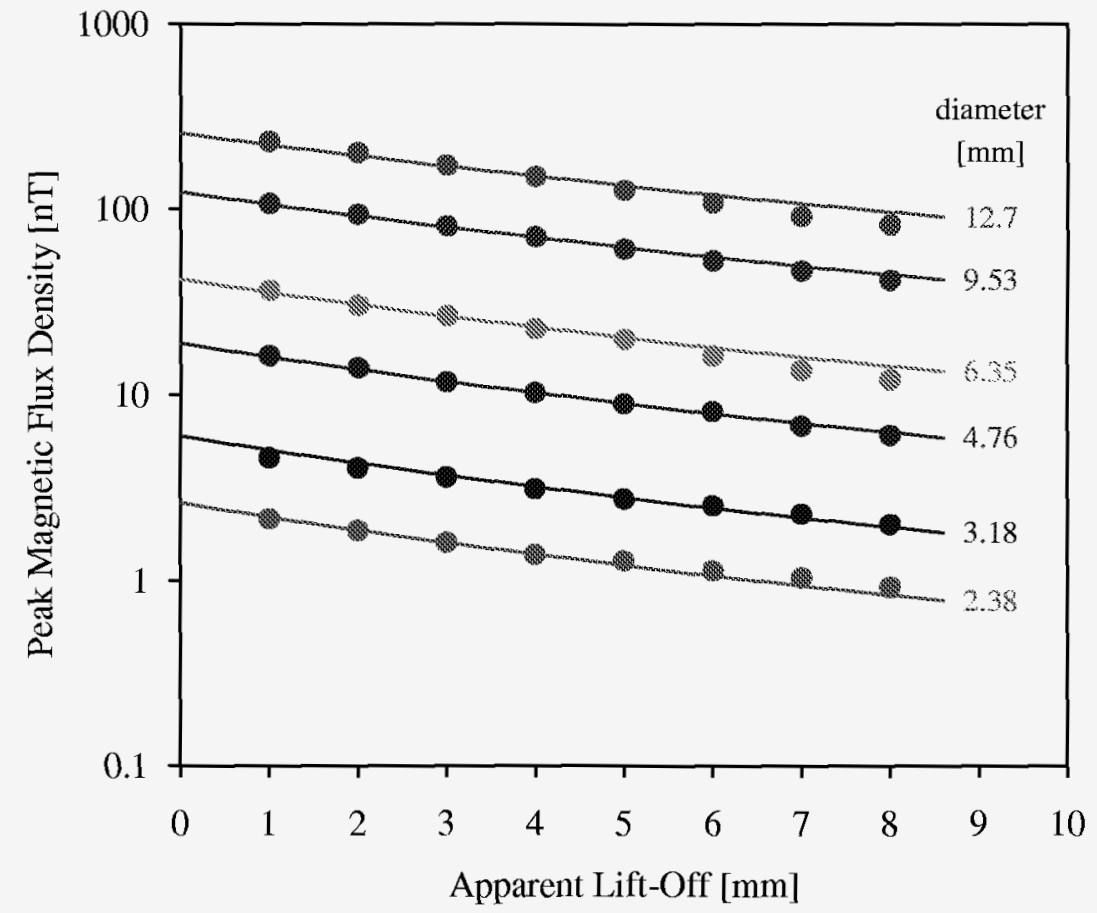

Figure 12 Comparison between the experimentally measured and theoretically predicted magnetic flux densities plotted as functions of the lift-off distance for surfacebreaking tin inclusions in copper $\left(\nabla T \approx 0.7^{\circ} \mathrm{C} / \mathrm{cm}\right)$.

Because of the decay of the magnetic field with increasing lift-off distance, the effective center of the sensing element is closer to the surface than its geometrical center. We tried to 
compensate the resulting inherent underestimation in the analytically predicted magnetic field by best fitting the numerical results to the experimental data using the unknown effective depth of the sensing element as the variable parameter. The least-mean-square fitting process yielded an optimal depth of $11.04 \mathrm{~mm}$, slightly lower than the previously described rough approximation based on the geometrical center of the sensing element, as one would expect. With this reasonable adjustment, the standard deviation between the theoretical predictions and the experimental results is only $6.3 \%$ (based on the geometrical center of the sensor, i.e., without any adjustable parameter, the corresponding error is approximately 18\%). The same results are plotted in a different way in Fig. 13a that compares the experimentally measured and theoretically predicted magnetic flux densities for all the different diameters and lift-off distances. Considering the rather crude approximations used in the theoretical model, the inherent uncertainties of the large number of independent material parameters involved in the phenomenon, and the potential experimental errors associated with the measurement, the agreement over a range of more than two orders of magnitude is surprisingly good, possibly even fortuitous to some degree.

Figure 13b shows our theoretical predictions and experimental results for the half-width of the magnetic signature as a function of lift-off. In both cases, good agreement was found between our theoretical predictions and experimental observations. Similar measurements were also conducted on subsurface inhomogeneities by simply turning the $12.7-\mathrm{mm}$-thick specimens upside-down. On the average, the measured magnetic signals decreased by roughly one order of magnitude, which is in good agreement with the expected reduction based on our theoretical model, but they were still readily detectable, which clearly indicates that buried imperfections can be also detected and characterized by the noncontacting thermoelectric method.

Another quantitative parameter that can be readily used to compare our analytical predictions and experimental observations is the half-width of the bi-polar magnetic signature, which was defined as half of the lateral separation between the positive and negative peaks. The theoretical curve for the normalized half-width as a function of the normalized lift-off was previously shown in Figure 8. Again, the differential arrangement of the gradiometer slightly complicates the comparison of the experimental data to the theoretical predictions. In the amplitude measurements, we simply subtracted the predicted magnetic field at the location of the secondary sensor from that of the primary sensor to account for the weak, but not entirely 
negligible secondary signal. Clearly, simple subtraction of the half-widths predicted at the levels of the primary and secondary sensors would not give the right answer, therefore we compared the experimental results simply to the predicted values at the level of the primary sensor. Figure $13 \mathrm{~b}$ shows our theoretical predictions (solid line) and experimental results (symbols) for the halfwidth of the magnetic signature as a function of lift-off. There is a good agreement between the theoretical and experimental data except when the normalized lift-off distance exceeds 10 . It should be mentioned that a large normalized lift-off results mainly from a small inclusion diameter, i.e., from the normalization process itself, rather than a large absolute lift-off. Since the peaks are not only smaller but also less sharp in the case of small inclusions, it is not surprising that the accuracy of the measured half-width also declines.

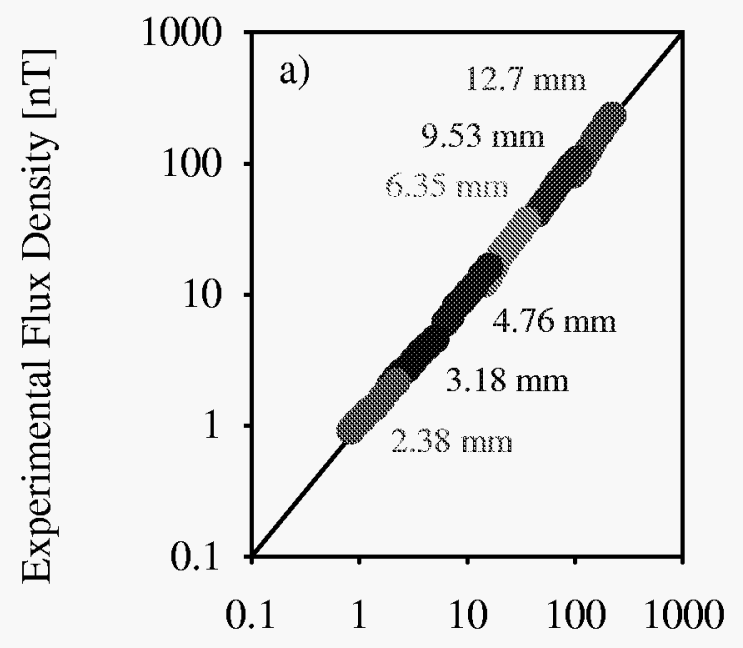

Theoretical Flux Density [nT]

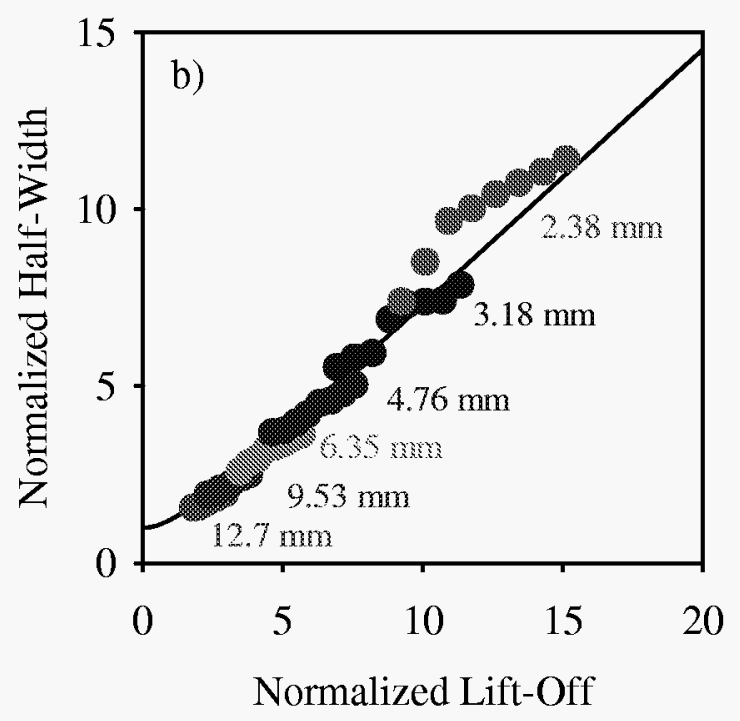

Figure 13 Comparison between the experimentally measured and theoretically predicted magnetic flux densities for surface-breaking tin inclusions in copper (a) and theoretical predictions (solid line) and experimental results (symbols) for the half-width of the magnetic signature as a function of lift-off (b).

In this part of our project, we conducted an experimental investigation of the magnetic field produced by thermoelectric currents around surface-breaking spherical tin inclusions in copper bars under external thermal excitation. The diameter of the inclusions and the lift-off distance varied from 2.38 to $12.7 \mathrm{~mm}$ and from 12 to $20 \mathrm{~mm}$, respectively. We enforced a 
constant $0.7^{\circ} \mathrm{C} / \mathrm{cm}$ temperature gradient in the specimen. The resulting peak magnetic flux densities ranged from 1 to $250 \mathrm{nT}$ and could be easily measured by a commercial fluxgate magnetometer. Our main goal was to verify the previously described theoretical predictions [10]. After appropriate normalization to facilitate such comparison, our experimental results were found to be in good quantitative agreement with the predictions of this model concerning both the absolute magnitude and the spatial distribution of the thermoelectric magnetic field [11]. These results clearly indicate that inclusions and other types of imperfections in metals can not only be nondestructively detected by noncontacting magnetic measurements, but can also be quantitatively characterized using the previously developed analytical model.

IV. Theoretical modeling of the signal from cylindrical and ellipsoidal inclusions

In Section II.1 we briefly reviewed our theoretical technique for isotropic spherical inclusions, which can be also used to predict the magnetic field produced by thermoelectric currents around isotropic cylindrical inclusions of circular cross section [10]. These models include only one geometrical parameter, namely the radius of the (either spherical or cylindrical) inclusion. However, real applications often involve inclusions of more complex geometries requiring more general models to predict the resulting thermoelectric signal. The next level of geometrical complexity, which is still analytically tractable, is the specific case of cylinders of elliptical cross section and ellipsoidal inclusions. These more complex models involve two geometrical parameters, namely the radius and the aspect ratio of the inclusion and the latter can be used to simulate a wider range of flaw shapes encountered in real applications. These models can be also used to study the effect of flaw orientation with respect to the dominant heat flux in the specimen under inspection. Whereas formal solutions for cylindrical and spherical inclusions could be obtained in forms of elementary functions that are widely available in textbooks, elliptical inclusions present considerably greater complexity. However, we recognized that classical solutions of similar potential problems in heat transfer [18], electricity [19], and hydrodynamics [20] are available for elliptical geometries. The governing equations of these problems closely resemble those of the uncoupled thermal conduction and electrical problems under consideration. These solutions, together with the methodology of dealing with the coupled equations of thermoelectricity, can be exploited to solve the problem under consideration. 


\section{IV.1 Analytical predictions}

First, let us consider an infinite, homogeneous, and isotropic medium containing a cylindrical inclusion of elliptical cross section aligned with the $z$-direction of a given Cartesian coordinate system $(x, y, z)$ as illustrated in Fig. 14a. The principal dimensions of the elliptical cross section are denoted by $2 a$ and $2 b$ along the $x$ and $y$ axes, respectively, while the system is subjected to a thermal excitation that would result in uniform temperature gradient along the $x$-axis in the absence of the inclusion. Like before, the magnetic field produced by thermoelectric currents flowing around the inclusion can be written in a normalized form of $H_{z}=H_{0} F_{z}(x, y)$, where the absolute magnitude of the field is [12]

$$
H_{0}=a \nabla T \sigma \Delta S \Gamma_{e} .
$$

Here, $\Gamma_{e}$ is a modified thermoelectric contrast for cylinders of elliptical cross section, which is a function of the electrical and thermal conductivity ratios between the inclusion and the host material as well as the aspect ratio $e=c / b$. In Eq. (26) we introduced the geometrical mean radius $a=\sqrt{b c}$ of the cylinder in order separate size and shape effects. This allows us to better compare the magnetic fields of cylindrical inclusions of identical cross sectional area, but different aspect ratio.

a)

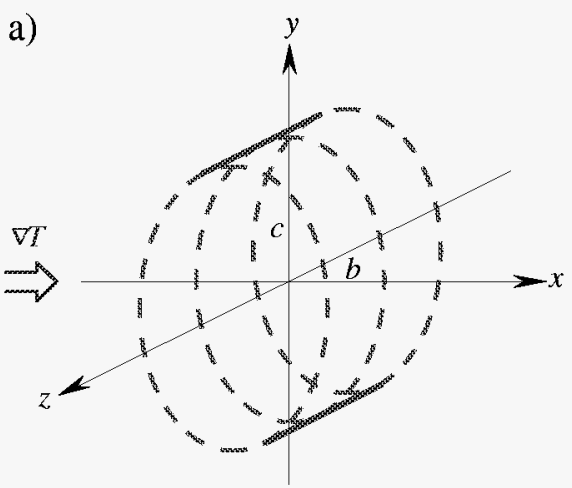

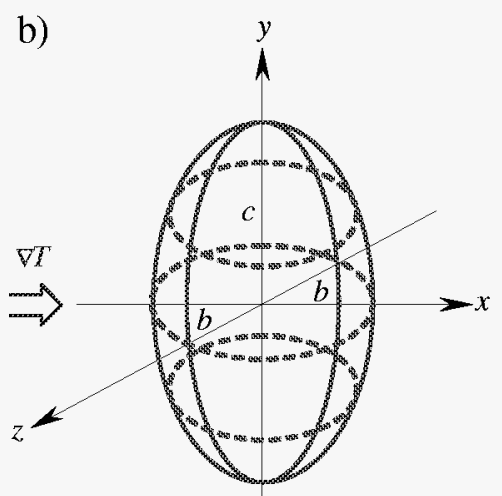

Figure 14 Schematic diagrams of (a) a cylindrical inclusion of elliptical cross section and (b) an ellipsoidal inclusion.

As an example, Fig. 15 shows the normalized thermoelectric contrast of a cylindrical inclusion of elliptical cross section relative to that of a cylinder with circular cross section and identical cross sectional area as a function of its aspect ratio assuming that the electrical and 
thermal conductivities of the inclusion are approximately $15 \%$ of those of the host material (for details, see Ref. 12).

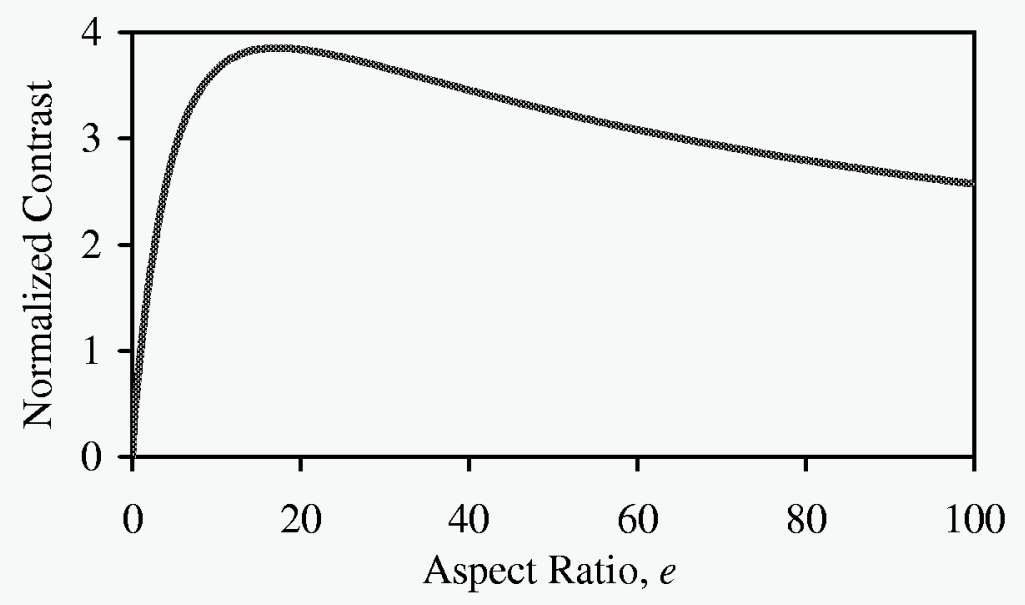

Figure 15 Normalized thermoelectric contrast of a cylindrical inclusion of elliptical cross section versus its aspect ratio assuming that the electrical and thermal conductivities of the inclusion are approximately $15 \%$ of those of the host material.

As expected, for a cylinder of circular cross section $(e=1)$ the results are identical to those of the simpler model developed in Ref. 10. For very low aspect ratios $(e \approx 0)$, i.e., when the cross section is elongated along the heat flow, the contrast vanishes. Similarly, when the aspect ratio increases $(e \rightarrow \infty)$, i.e., when the cross section is elongated in a direction normal to the principal heat flow, the contrast also vanishes, although much slower. It is easy to see that in both limiting cases the disturbance caused by a cylindrical inclusion of constant cross sectional area will diminish. Interestingly, the maximum thermoelectric contrast is produced by an elliptical cross section that is moderately elongated $(e \approx 10)$ normal to the heat flow, in which case the thermoelectric signal is approximately four times higher than that of the corresponding cylindrical inclusion of circular cross section.

We have conducted similar calculations for an infinite, homogeneous, and isotropic medium containing an ellipsoidal inclusion as illustrated in Fig. 14b. Although a more general spheroidal inclusion of three different principal dimensions could be modeled in a similar fashion, the analytical solution would become much more cumbersome. We found that in the 
case of an ellipsoidal inclusion the thermoelectric contrast retains the same functional dependence on the aspect ratio as in the case of a cylindrical inclusion with elliptical cross section, though the spatial distribution of the magnetic field $F(x)$ is obviously different since it will have components in both the $y$ and $z$ directions as opposed to the cylindrical case when the magnetic field was strictly parallel to the $z$-axis. Again, for a spherical inclusion $(e=1)$ the results for an ellipsoidal inclusion are identical to those of the simpler model [10].

In conclusion, due to the difference in the thermoelectric properties of the host and the inclusion, the temperature and electric field distributions will be distorted accordingly to the geometry of the inclusion, therefore the thermoelectric signal produced by an inclusion will depend not only on its material properties and size, but also on its shape. Our results indicate that elongated inclusions can be best detected by aligning the externally enforced temperature gradient normal to the major dimension of the inclusion. Further experimental efforts are needed to verify these theoretical predictions and to develop inversion methods capable of quantitatively evaluating the measured magnetic signals in terms of size and shape for inclusions of known physical properties. These efforts will be discussed in more detail in the proposal following this report.

V Thermoelectric background signature produced by anisotropic materials

As we have already pointed it out, the threshold sensitivity of the noncontacting thermoelectric method is ultimately limited by the temporally coherent material "noise" or "background signature" that cannot be eliminated by time-averaging. This material signature is usually produced by macrostructural features, such as heat-affected, work-hardened, strained, and textured regions. Let us consider an example of how this material signature could limit the detectability of weak imperfections by the thermoelectric method. Let us consider the characterization of weak surface and near-surface imperfections caused by localized plastic deformation that is usually very difficult to detect by conventional NDE methods because they are effectively hidden by the accompanying surface roughness. A good example is the characterization of near-surface material damage on fretted Ti-6Al-4V specimens. Figure 16 shows the magnetic scans of two Ti-6Al-4V specimens taken at $\nabla T \approx 13{ }^{\circ} \mathrm{C} / \mathrm{cm}$ temperature gradient for two opposite heating directions. The specimen without fretting damage exhibits a 
background signature of $\approx 7 \mathrm{nT}$ peak-to-peak amplitude that is caused by intrinsic anisotropic texture. After substantial fretting damage was induced over a $\approx 12$-mm- diameter spot at the center (as indicated schematically by the superimposed circle), the magnetic signature essentially doubled to $\approx 14 \mathrm{nT}$. The main advantage of the thermoelectric method is that it is sensitive only to the "material" effects of the damage, namely residual stress, local texture, and increased dislocation density, but it is entirely insensitive to its geometrical by-product, i.e., the rough surface topography produced by fretting wear. However, the detectability of weak imperfections is obviously adversely affected by the presence of the rather strange looking background signature in the intact material, that can be reduced only by annealing above the recrystallization temperature, which would also completely change the existing microstructure.

a) before fretting (some texture)
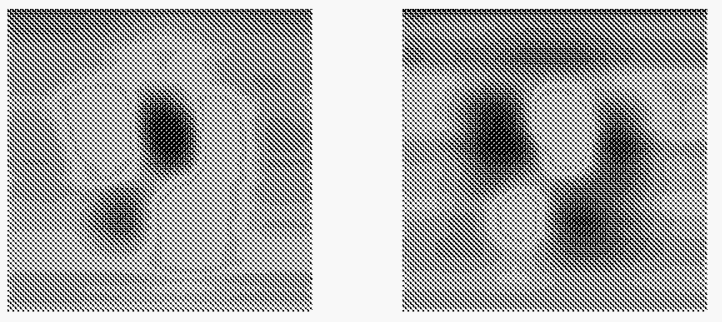

b) after fretting ( $25 \mathrm{~min}$ )
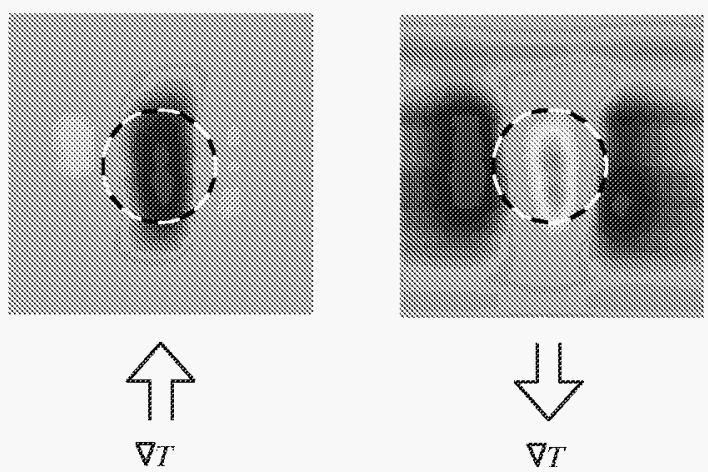

Figure 16 Magnetic scans of a Ti-6Al-4V specimen before and after fretting $\left(\nabla T \approx 13^{\circ} \mathrm{C} / \mathrm{cm}\right.$ temperature gradient, $3 " \times 3 "$ ).

The appearance of the above described background signature in noncontacting thermoelectric inspection is not surprising at all. It has been known for a long time that pure cold-rolled metals can exhibit a rather significant variation of $\approx 1 \%$ in thermoelectric power with respect to the same metal in annealed condition [21]. Texture induced anisotropy can also 
lead to significant thermoelectric signals between regions of different orientations in the same material $[4,22]$. Many manufacturing processes produce a textured microstructure that causes a perceivable macroscopic anisotropy in the material properties. The nature of this texture can by crystallographic, e.g., preferred orientation of non-cubic grains in a polycrystalline metal [23], or morphological, e.g., preferred orientation of grain boundaries, dislocations, slip bands, segregations, etc. In addition, the presence of strong residual stresses can also cause weak spurious anisotropy in otherwise isotropic materials via the substantial stress-dependence of the thermoelectric properties.

\section{V.1 Analytical predictions}

In the simplest case, the material properties of the anisotropic medium are homogeneous, i.e., they are the same at every point, but they change perceivably with orientation. A significant thermoelectric signature arises only when the direction of the heat flux rapidly changes from point to point due to either nonuniform heating and cooling or the irregular shape of the specimen. The former effect is caused by localized heating and cooling used to introduce the necessary temperature gradient in the specimen. In order to demonstrate how anisotropy can induce a nonvanishing thermoelectric current distribution in a specimen under forced heating and cooling, let us first consider an infinite line source acting in an infinite anisotropic medium. Since all material properties are affected by the same microstructural symmetry, we are going to assume that the electrical conductivity $\sigma$, thermal conductivity $\boldsymbol{\kappa}$, and thermoelectric power $S$ tensors all exhibit the same principal directions, though their degree of anisotropy might be very different. In order to further simplify the calculations, we are going to assume that the line source is oriented along one of the principal directions of the medium, that is aligned with the $x_{3}$-axis of a Cartesian principal coordinate system. In this way, the problem becomes essentially two-dimensional with both the heat flux and the thermoelectric current limited to the $x_{1}, x_{2}$ plane of symmetry and exact analytical solutions can be obtained for the thermoelectric current distribution and the induced magnetic field [13].

In order to satisfy our two field equations, $\nabla \cdot \mathrm{j}=0$ and $\nabla \cdot \mathrm{h}=0$, we seek solutions in the following forms: 


$$
T=B \ln \left[\frac{x_{1}^{2}}{\beta_{1}}+\frac{x_{2}^{2}}{\beta_{2}}\right] \text { and } \Phi=D \ln \left[\frac{x_{1}^{2}}{\beta_{1}}+\frac{x_{2}^{2}}{\beta_{2}}\right] \text {. }
$$

Here, $B$ and $D$ are currently unknown amplitudes that will be later determined from the appropriate boundary conditions and $\beta_{1}$ and $\beta_{2}$ are diffusion coefficients determined by the anisotropic material properties of the medium. The characteristic equation for $\beta_{1}$ and $\beta_{2}$ can be obtained from the field equations and the tensorial form of the constitutive relations formerly given as a scalar relationship in Eq. (1)

$$
\left[\begin{array}{ll}
\frac{\varepsilon_{1}}{\beta_{1}}-\frac{\varepsilon_{2}}{\beta_{2}} & \frac{\sigma_{1}}{\beta_{1}}-\frac{\sigma_{2}}{\beta_{2}} \\
\frac{\kappa_{1}}{\beta_{1}}-\frac{\kappa_{2}}{\beta_{2}} & \frac{\bar{\varepsilon}_{1}}{\beta_{1}}-\frac{\bar{\varepsilon}_{2}}{\beta_{2}}
\end{array}\right]\left[\begin{array}{l}
B \\
D
\end{array}\right]=\left[\begin{array}{l}
0 \\
0
\end{array}\right] .
$$

Nontrivial solutions are found by first solving the eigenvalue problem and then determining the corresponding eigenvectors. For brevity, we are going to introduce the following notation for the ratios of the diffusion coefficients and material properties; $\beta=\beta_{1} / \beta_{2}$, $\sigma=\sigma_{1} / \sigma_{2}, \quad \varepsilon=\varepsilon_{1} / \varepsilon_{2}, \quad \kappa=\kappa_{1} / \kappa_{2}$. Furthermore, $\eta=\left(\varepsilon_{2} \bar{\varepsilon}_{2}\right) /\left(\sigma_{2} \kappa_{2}\right)=\sigma_{2} S_{2}^{2} T / \kappa_{2}$ will be used without a subscript to denote the thermoelectric coupling of the material in the second principal direction. The sought characteristic equation for $\beta$ is obtained by requiring that the determinant of the characteristic matrix in Eq. (28) vanish

$$
\eta(\varepsilon-\beta)^{2}-(\sigma-\beta)(\kappa-\beta)=0 .
$$

This quadratic equation yields two eigenvalues that are denoted by $\beta_{p}$ and $\beta_{n}$

$$
\beta_{p, n}=\frac{\sigma+\kappa-2 \varepsilon \eta \pm \sqrt{(\sigma-\kappa)^{2}-4 \eta(\sigma-\varepsilon)(\varepsilon-\kappa)}}{2(1-\eta)}
$$

where subscripts $p$ and $n$ correspond to the positive and negative values of the square root in the numerator. Finally, the corresponding eigenvectors can be determined from either rows of Eq. (28) as follows 


$$
\delta_{p, n}=\frac{D_{p, n}}{B_{p, n}}=-\frac{\varepsilon_{2}\left(\varepsilon-\beta_{p, n}\right)}{\sigma_{2}\left(\sigma-\beta_{p, n}\right)} .
$$

Formal solution for the electrical current density and heat flux can be obtained from these temperature and electrical potential distributions by substituting them into the constitutive equation. After some algebraic manipulation, the electrical current density and thermal flux vectors are obtained as

$$
\mathrm{j}=-2\left(\frac{B_{p} \gamma_{p}}{\frac{x_{1}^{2}}{\beta_{p}}+x_{2}^{2}}+\frac{B_{n} \gamma_{n}}{\frac{x_{1}^{2}}{\beta_{n}}+x_{2}^{2}}\right)\left(x_{1} \mathrm{e}_{1}+x_{2} \mathrm{e}_{2}\right)
$$

and

$$
\mathrm{h}=-2\left(\frac{B_{p} v_{p}}{\frac{x_{1}^{2}}{\beta_{p}}+x_{2}^{2}}+\frac{B_{n} v_{n}}{\frac{x_{1}^{2}}{\beta_{n}}+x_{2}^{2}}\right)\left(x_{1} \mathrm{e}_{1}+x_{2} \mathrm{e}_{2}\right),
$$

where $\mathrm{e}_{1}$ and $\mathrm{e}_{2}$ are unit vectors along the $x_{1}$ and $x_{2}$ coordinate axes, respectively, and

$$
\gamma_{p, n}=\varepsilon_{2} \frac{\sigma-\varepsilon}{\sigma-\beta_{p, n}} \quad \text { and } \quad v_{p, n}=\kappa_{2}\left(1-\eta \frac{\varepsilon-\beta_{p, n}}{\sigma-\beta_{p, n}}\right)
$$

The temperature and electric potential distributions can be written by extending Eq. (27) as follows

$$
T=B_{p} \ln \left[\frac{x_{1}^{2}}{\beta_{p}}+x_{2}^{2}\right]+B_{n} \ln \left[\frac{x_{1}^{2}}{\beta_{n}}+x_{2}^{2}\right]
$$

and

$$
\Phi=B_{p} \delta_{p} \ln \left[\frac{x_{1}^{2}}{\beta_{p}}+x_{2}^{2}\right]+B_{n} \delta_{n} \ln \left[\frac{x_{1}^{2}}{\beta_{n}}+x_{2}^{2}\right]
$$


Eqs. (32) through (36) are general solutions of the coupled field equations under the assumption that the total heat evolving per unit volume can be approximated by the divergence of the heat flux, i.e., thermoelectric contributions (Peltier and Johnson heat) as well as the Joule heat produced by the weak thermoelectric current are negligible. The only remaining unknowns, $B_{p}$ and $B_{n}$, can be readily determined from the boundary conditions prevailing around the line source at $x_{3}$. By integrating the electrical current density and heat flux, we get

$$
-4 \pi \gamma_{p} \sqrt{\beta_{p}} B_{p}-4 \pi \gamma_{n} \sqrt{\beta_{n}} B_{n}=0
$$

and

$$
-4 \pi v_{p} \sqrt{\beta_{p}} B_{p}-4 \pi v_{n} \sqrt{\beta_{n}} B_{n}=\dot{Q}
$$

respectively, where $\dot{Q}$ denotes the total heat power emanating from a unit length of the line source. These equations can be solved for $B_{n}$ and $B_{p}$ as follows

$$
B_{p}=\frac{\dot{Q}}{4 \pi \sqrt{\beta_{p}}\left(\frac{\gamma_{p}}{\gamma_{n}} v_{n}-v_{p}\right)} \text { and } B_{n}=\frac{\dot{Q}}{4 \pi \sqrt{\beta_{n}}\left(\frac{\gamma_{n}}{\gamma_{p}} v_{p}-v_{n}\right)}
$$

Finally, the sought magnetic field can be obtained from Maxwell's equation of $\nabla \times \mathrm{H}=\mathrm{j}$ by integration. The only nonvanishing component is parallel to the line source

$$
H_{z}=\frac{\dot{Q}}{2 \pi} \frac{\tan ^{-1}\left(\sqrt{\beta_{p}} \frac{x_{2}}{x_{1}}\right)-\tan ^{-1}\left(\sqrt{\beta_{n}} \frac{x_{2}}{x_{1}}\right)}{\frac{v_{p}}{\gamma_{p}}-\frac{v_{n}}{\gamma_{n}}} .
$$

It is clear that there is no magnetic field along any of the principal directions $\left(x_{1}=0\right.$ or $x_{2}=0$ ), i.e., where the material appears to be "quasi-isotropic" in the sense that the corresponding components of the electrical current density and heat flux are parallel to the gradients of the temperature and electrical potential. 
It should be mentioned that the degree of anisotropy in thermal $\kappa$, electrical $\sigma$, and thermoelectric $\varepsilon$ properties is much smaller than the corresponding degree of anisotropy in mechanical properties. First of all, crystallographic anisotropy is limited to noncubic materials only, which effectively excludes all of the important structural metals such as aluminum, steel, nickel, copper, etc. Even in noncubic metals like titanium, which preferentially crystallizes in hexagonal symmetry, the crystallographic anisotropy is only a few percents. In most cases, anisotropy is caused by relatively weak morphological features of texture, e.g., the evolution of elongated grain shapes in the direction of plastic deformation during cold working. Although, the thermal and electrical properties are perceivably affected by such texturing in the material, the resulting degree of anisotropy is usually rather modest. Therefore, a very useful approximation can be made by assuming that the degree of anisotropy is small $(0.9<\sigma, \kappa, \varepsilon<1.1)$, so that both $\beta_{p}$ and $\beta_{n}$ are close to unity and consequently Eq. (40) can be approximated by

$$
H_{z} \approx \frac{\dot{Q}}{2 \pi} \frac{\sqrt{\beta_{p}}-\sqrt{\beta_{n}}}{\frac{v_{p}}{\gamma_{p}}-\frac{v_{n}}{\gamma_{n}}} \frac{x_{1} x_{2}}{x_{1}^{2}+x_{2}^{2}}=H_{0} F\left(x_{1}, x_{2}\right)
$$

that allows a much clearer separation of the parameters affecting the strength of the magnetic field, $H_{0}$, and a simplified universal spatial distribution

$$
F\left(x_{1}, x_{2}\right)=\frac{x_{1} x_{2}}{x_{1}^{2}+x_{2}^{2}}
$$

$H_{0}$ is a fairly complicated function of the material properties via Eqs. (30) and (34), but it does vanish when the degree of anisotropy diminishes, i.e., when $\sigma, \kappa$, and $\varepsilon$ all approach unity. The magnetic field depends only on the angular coordinate of the point of observation with respect to the principal directions but not on the distance from the source. In contrast to the electrical current density and heat flux, which are inversely proportional to the distance from the source, the magnetic field produced by an infinite line source exhibits no loss associated with the spreading of the heat. It should be mentioned again that $F\left(x_{1}, x_{2}\right)$ vanishes on the principal axes $\left(x_{1}=0\right.$ or $\left.x_{2}=0\right)$ where there is no skewing between $\nabla \Phi$ and $\nabla T$ on one side and $\mathrm{j}$ and $\mathrm{h}$ on the other side. 


\section{V.2 Infinite dipole along a principal direction}

In order to illustrate the main features of our analytical predictions for the anisotropic thermoelectric effect in the case of simultaneous heating and cooling, let us consider the case of a dipole consisting of a line source and a sink separated by a unit distance $b=1$ as it is shown in Fig. 17. Because of the cancellation effect between the source and the sink, far away from the dipole the magnetic field decreases as the inverse of the distance from the dipole. Now, the principal directions of the anisotropic material $\left(x_{1}, x_{2}\right)$ are rotated by an arbitrary angle of $\theta$ with respect to the dipole orientation along the $x_{3}=\xi_{3}$ axis. Using superposition, the magnetic field of the dipole can be written in the physical coordinate system $\left(\xi_{1}, \xi_{2}, \xi_{3}\right)$ as $H_{z}=H_{0} F_{d}\left(\xi_{1}, \xi_{2}\right)$, where

$$
F_{d}\left(\xi_{1}, \xi_{2}\right)=F_{r}\left(\xi_{1}, \xi_{2}+0.5\right)-F_{r}\left(\xi_{1}, \xi_{2}-0.5\right)
$$

Here, $F_{r}\left(\xi_{1}, \xi_{2}\right)$ denotes the rotated distribution function of a line source, that can be easily calculated from the previously determined approximate spatial distribution function $F\left(x_{1}, x_{2}\right)$ by rotation from the material coordinate system $\left(x_{1}, x_{2}\right)$ into the physical coordinate system $\left(\xi_{1}, \xi_{2}\right)$, i.e., by substituting $x_{1}=\xi_{1} \cos \theta+\xi_{2} \sin \theta$ and $x_{2}=-\xi_{1} \sin \theta+\xi_{2} \cos \theta$. For simplicity, in Eq. (43) we normalized all coordinates to the separation distance $b$ between the source and the sink.

Figures $18 \mathrm{a}$ and $18 \mathrm{~b}$ show the normalized magnetic field distributions $F_{d}\left(\xi_{1}, \xi_{2}\right)$ of the dipole shown in Fig. 17 for $\theta=0^{\circ}$ and $\theta=45^{\circ}$, respectively. When the dipole is aligned with the principal directions of the material $\left(\theta=0^{\circ}\right)$, two large asymmetric lobes of opposite signs appear on the two sides of the dipole direction. Since the main heat flux is along the dipole direction from the source towards the sink, this type of material signature is similar to the lobes produced by inclusions. When the dipole is oriented along the bisector between the principal directions of the material $\left(\theta=45^{\circ}\right)$, both main lobes split into two twin peaks and the distribution becomes symmetric with respect to the dipole direction. This type of material signature is rather unique and quite different from the typical pattern produced by inclusions. As we mentioned earlier, far away from the dipole the source and the sink increasingly cancel each other and the magnetic field is inversely proportional to distance. 


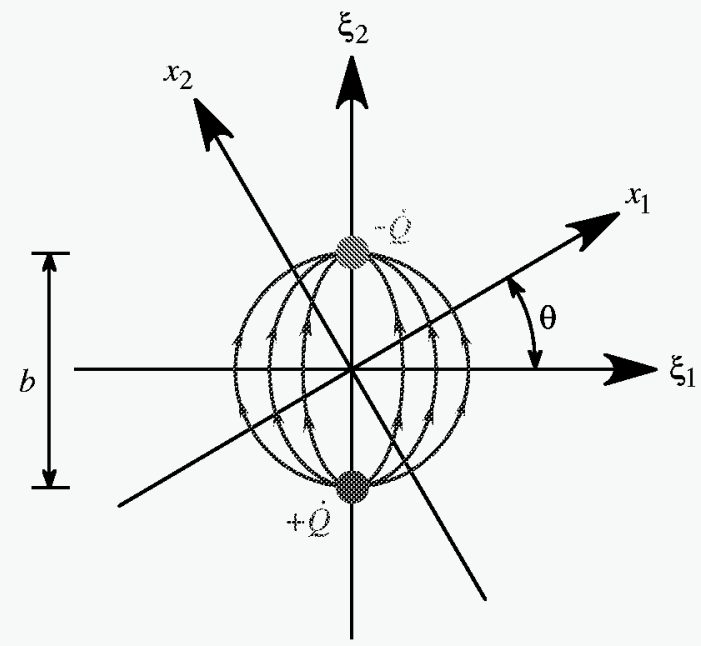

Figure 17 A schematic diagram of a dipole consisting of a line source and a sink separated by a unit distance $b=1$.

a) $\theta=0^{\circ}$

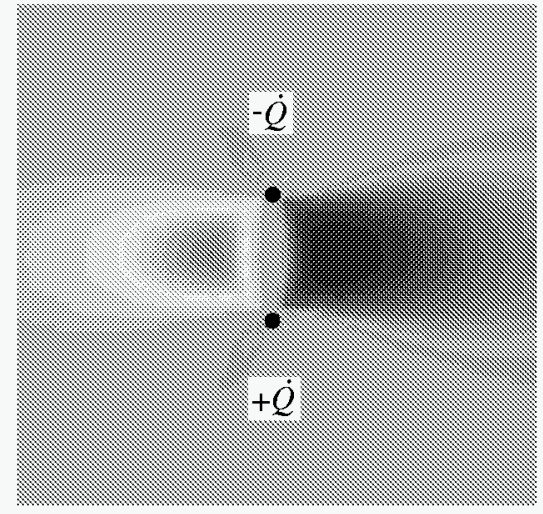

b) $\theta=45^{\circ}$

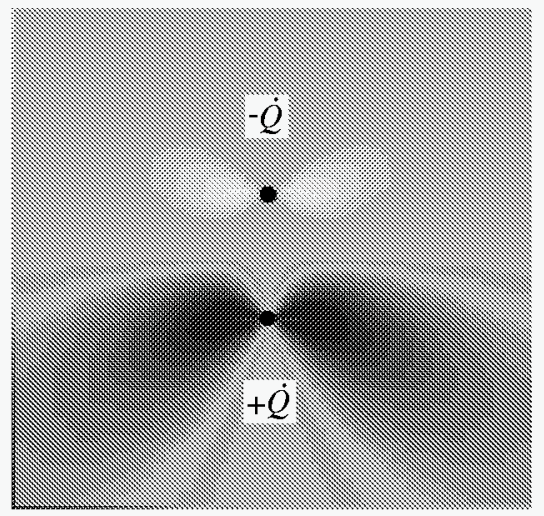

Figure 18 Normalized magnetic field distributions of the dipole shown in Fig. 17 in an anisotropic material for (a) $\theta=0^{\circ}$ and (b) $\theta=45^{\circ}$. 


\section{V.3 Experimental verification}

In order to qualitatively verify the previously described analytical model, we conducted a series of experiments on a 1.9-mm-thick cold-rolled Ti-6Al-4V plate. First, we determined the principal material directions in the highly textured plate using an elliptical eddy current coil of $5: 1$ aspect ratio at $100 \mathrm{kHz}$. We found that the anisotropy of the electrical conductivity, i.e., the relative difference between the principal directions, was approximately $2.3 \%$. We also measured the degree of thermoelectric anisotropy using an ATS-6044 Alloy ThermoSorter (Walker Scientific, Inc.) which allows directional measurements in a thin plate. First, the equipment was calibrated on reference materials of known absolute thermoelectric power. At each orientation, the thermoelectric power was measured at 200 randomly chosen locations and averaged to get a representative value. Figure 19 shows the measured orientation-dependence of the absolute thermoelectric power in the cold-rolled Ti-6Al-4V specimen (symbols) along with the bestfitting sinusoidal curve (solid line). The relative anisotropy of the thermoelectric power was approximately $3.1 \%$, somewhat higher than that of the electrical conductivity. Our limited goal is simply to qualitatively verify our theoretical model by comparing the rather strange spatial distributions of the analytical predictions and experimental observations, therefore the less easily measurable anisotropy factor of the thermal conductivity was not determined at this point, but it is expected to be close to that of the electrical conductivity.

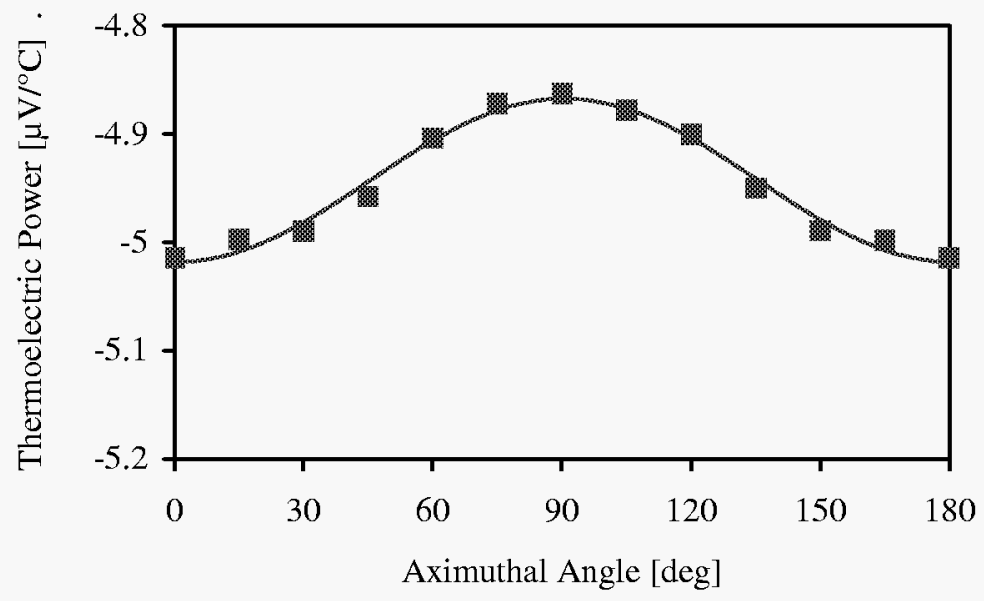

Figure 19 The orientation-dependence of the absolute thermoelectric power in a cold-rolled Ti-6Al-4V plate. 
Figure 20 shows a schematic diagram of the experimental arrangement used to map the spatial distribution of the magnetic signature. The experimental data presented here is actually the difference between measurements taken at opposite heating directions and then divided by two. In order to improve heat conduction from the 12-mm-diameter copper conductor rods to the specimen, a heat conducting silicone compound was applied between them. The centers of the heating and cooling spots was $50 \mathrm{~mm}$ apart and the temperature there was kept constant at $50{ }^{\circ} \mathrm{C}$ and $10{ }^{\circ} \mathrm{C}$, respectively. Because of its particular importance in interpreting the measured data, we should mention that, in order to eliminate the strong magnetic field of the Earth, we used ac coupling with a high-pass filter of very low cut-off frequency at $0.01 \mathrm{~Hz}$. The pseudo-dynamic magnetic signals required for ac detection were produced by laterally (normal to the main heat flux) scanning the specimen at a speed of $\approx 20 \mathrm{~mm} / \mathrm{s}$. In addition to the relatively fast lateral scanning in the "line" direction, we also scanned the specimens at a much lower rate in the axial "frame" direction, i.e., parallel to the main heat flux. In this way, a $203 \mathrm{~mm} \times 203 \mathrm{~mm}$ scan of $200 \times 200$ grid points took about 45 minutes. Whenever the magnetic field is asymmetric to the direction of heating, the resulting bipolar line signal does not exhibit a significant dc component and will be recorded without substantial distortion. However, if the magnetic field is symmetric to the direction of heating, the resulting unipolar line signal looses its dc component and will be significantly distorted.

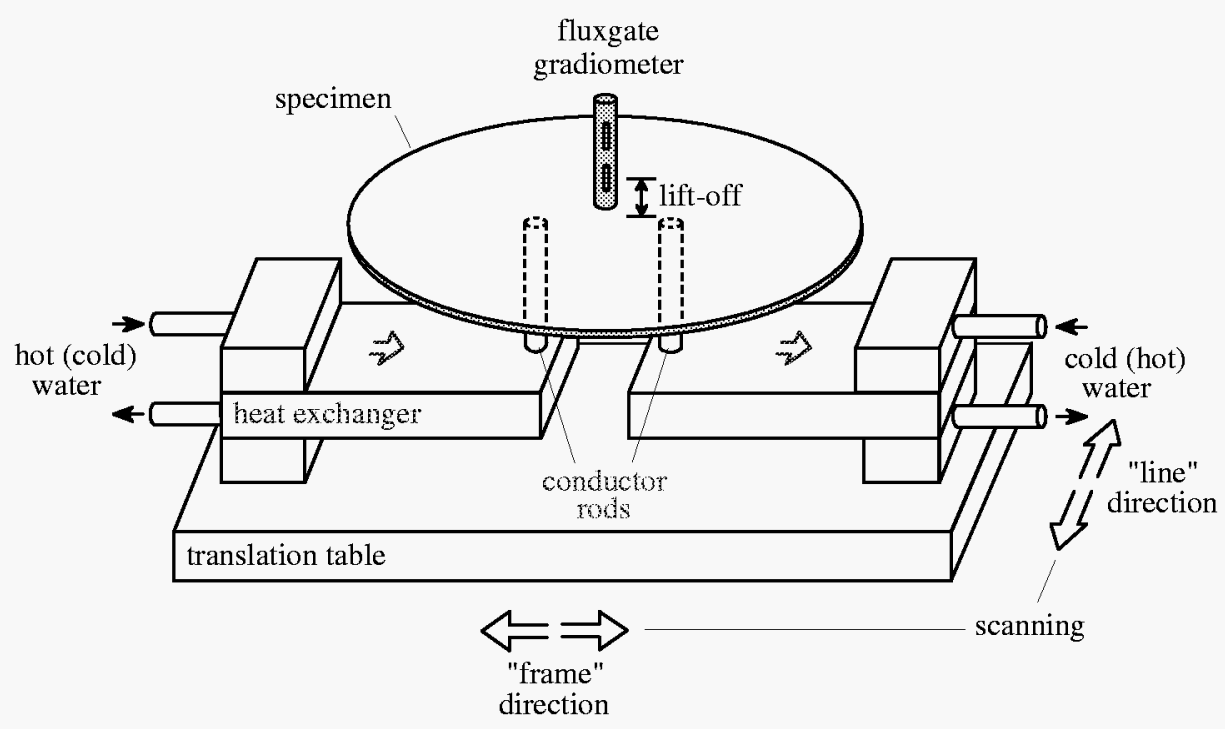

Figure 20 A schematic diagram of the experimental arrangement. 
Figure 21 shows the comparison between the theoretical and experimental results for the two-dimensional distribution of the magnetic signature produced by a cold-rolled Ti-6Al-4V titanium-alloy plate at four different orientations. Whenever $\theta$ is close to either $0^{\circ}$ or $90^{\circ}$, i.e., the principal material directions are aligned with the heating/cooling direction, two large asymmetric lobes of opposite signs appear on the two sides. In this case, the resulting line scans are bipolar in nature and are therefore well reproduced by the experimental data. However, when the dipole is aligned along the bisectors between the principal directions of the material, the main lobes split into twin peaks and the distribution becomes symmetric with respect to the dipole direction. As we mentioned above, these distributions are inherently distorted by the necessity of using ac coupling. This distortion effectively eliminates the average signal in each line scan thereby producing virtual peaks and valleys of opposite sign with respect to the dominating principal features. As a result, the measured distributions exhibit additional secondary bumps not predicted by the theory.

Considering the inherently lower resolution and ac distortion of the experimental images, the agreement with our theoretical predictions is very good, which clearly indicates that the suggested analytical model truthfully captures the main features of the anisotropic magnetic signature of textured materials. These results demonstrate that the earlier observed strange background signatures in textured specimens can be attributed to the thermoelectric anisotropy of the material. In such specimens the best flaw detectability can be achieved by rotating the heating/cooling direction so that the anisotropic effect averages out. The most important feature of the anisotropic material signature is that it changes sign when the specimen is rotated by $90^{\circ}$, but it always remains the same when the specimen is rotated by $180^{\circ}$. We will see later, that when the material signature is caused by material inhomogeneity, the signal changes sign for certain rotations of $180^{\circ}$, which can be exploited to distinguish between signatures due to anisotropy and inhomogeneity. Only noncubic materials such as titanium alloys exhibit crystallographic anisotropy, though a much weaker morphological anisotropy can be also exhibited by textured cubic materials. The results of this study can be used to optimize thermoelectric inspection procedures in textured metals. Furthermore, they also indicate that noncontacting thermoelectric inspection can be used to characterize the macroscopic texture of materials by evaluating their magnetic signatures under external heating and cooling. 


$$
\theta=0^{\circ}
$$
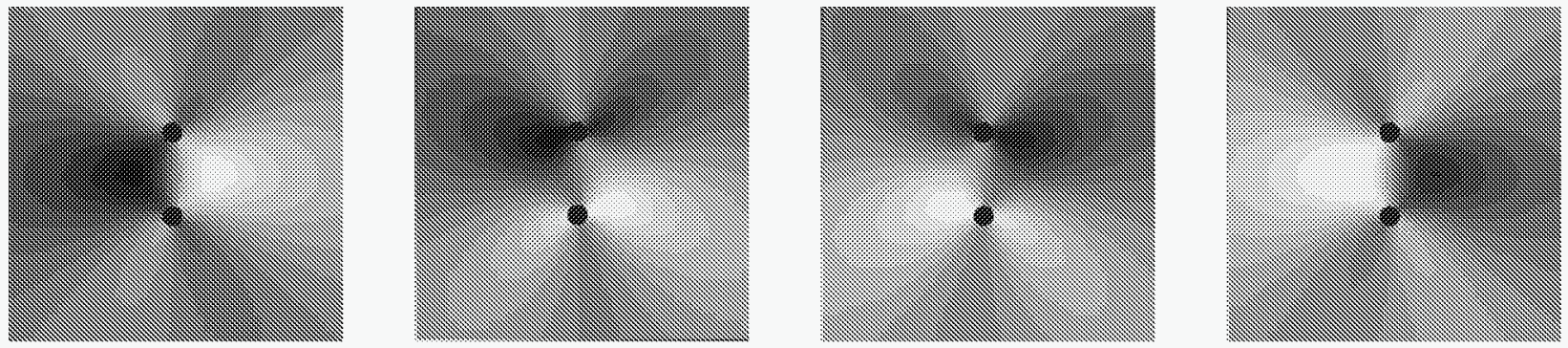

experimental
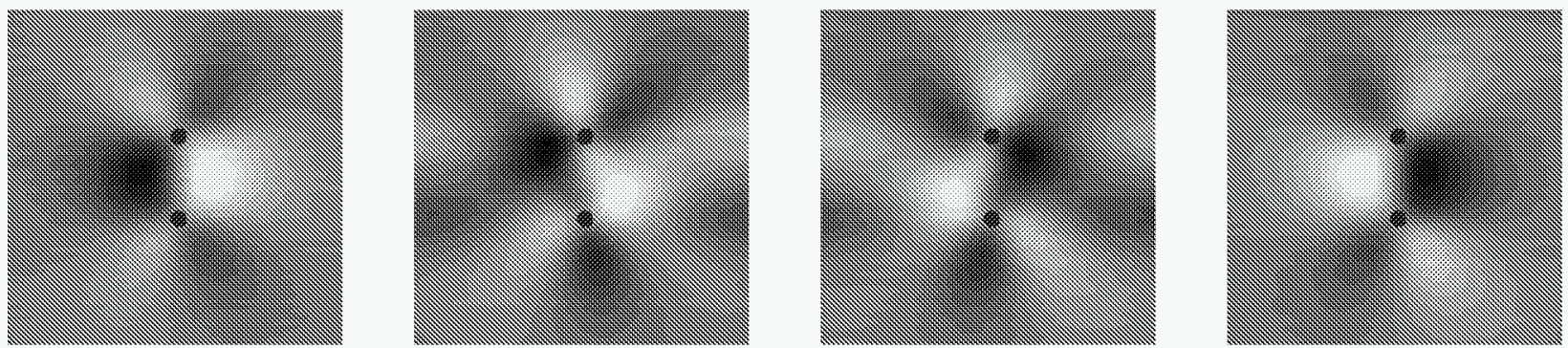

Figure 21 Comparison between our analytical and experimental results for the twodimensional distribution of the magnetic signature produced by an anisotropic Ti$6 \mathrm{Al}-4 \mathrm{~V}$ plate at $12-\mathrm{mm}$ lift-off (the superimposed spots indicate the heating and cooling locations).

VI Thermoelectric flaw signals produced by cracks and voids in anisotropic hosts

A second type of anisotropic signature occurs as a localized artifact caused by sharp geometrical features such as corners and rivet holes [14]. In isotropic materials, the noncontacting thermoelectric technique is sensitive to intrinsic material variations only regardless of the size, shape, and surface quality of the specimen to be tested. In anisotropic materials, this perfect separation between intrinsic material properties and extrinsic geometrical features does not exist anymore. For example, a cylindrical hole or an ellipsoidal cavity will produce a nonvanishing thermoelectric current distribution and a resulting magnetic field. Theoretically, this effect could be exploited to detect otherwise hidden cracks and voids in textured materials. However, the 
reliability of this indirect detection leaves much to be desired since the sensitivity strongly depends on the degree and orientation of the material anisotropy, which are usually unrelated to the presence of the sought discontinuity. On the other hand, the same signal could act like a spurious artifact that reduces the detectability of subtle material variations, such as the presence of fatigue damage, plastic deformation, or residual stresses, in the vicinity of stress concentrators.

\section{VI.1 Analytical predictions}

Based on the same tensorial approach used in the previous section to study global effects of anisotropy, we have developed an analytical model to predict the thermoelectric signature of cracks and voids in anisotropic hosts. Because of the complexity of combining the anisotropic constitutive relationships and the boundary conditions at material discontinuities, it was expected that explicit analytical results could not be obtained even in the simplest canonical cases. However, we found that appropriate coordinate transformations lead to relatively simple explicit solutions that can be further generalized to include such cases as anisotropic inclusions in homogeneous materials.

In order to illustrate the effect of material discontinuities in a textured host, let us consider an infinite isotropic cylindrical inclusion of radius $a$ embedded in an anisotropic medium whose physical properties exhibit the specific anisotropy described in the previous section, i.e., the principal directions of the electrical conductivity, thermal conductivity, and thermoelectric power are aligned with each other, but the relative degree of anisotropy of these parameters is not necessarily the same. Once again, the system is subjected to an externally enforced temperature gradient of $\nabla T$ along the $x_{1}$ direction. In order to simplify the calculations, we will assume that the inclusion is oriented along the $x_{3}$ principal direction of the medium. In this way, the problem becomes essentially two-dimensional with both the heat flux and the thermoelectric current limited to the $x_{1}, x_{2}$ plane of symmetry. Under these conditions, formal solutions for the temperature and electrical potential distributions can be obtained in the same way as in the previous section. Satisfying the boundary conditions is made more complicated by the fact that the heat flow around the inclusion will be disturbed in a way that depends on both the degree of material anisotropy in the host and the shape of the inclusion. 
However, this complexity can be essentially eliminated by appropriate coordinate transformations. For example, the problem of a cylindrical inclusion of circular cross section in an anisotropic host can be shown to be equivalent to that of a cylindrical inclusion of elliptical cross section in an isotropic host. The latter problem was solved in Ref. 12, which has been briefly reviewed Section IV. In the general case of a cylindrical inclusion of elliptical cross section in an anisotropic host, the virtual aspect ratio of the equivalent cylindrical inclusion of elliptical cross section in a virtual isotropic host can be calculated from the actual aspect ratio of the inclusion and the anisotropic parameters of the actual host.

As the simplest example of how the presence of anisotropy in the host material can expose the nonuniform heat flux in the vicinity of material discontinuities, Fig. 22 shows the spatial distribution of the thermoelectric magnetic field around a cylindrical hole of circular cross section. In absence of anisotropy the purely geometrical discontinuity would not produce any thermoelectric current and therefore no magnetic signal would be detected [10]. In the presence of anisotropy however a purely geometrical discontinuity also produces a thermoelectric magnetic signature. The spatial distribution of this signature is determined by the relative orientation of the heat flow with respect to the principal directions in the anisotropic host, while its magnitude mainly depends on the degree of anisotropy in the host. In order to illustrate the latter effect, Fig. 23 shows the variation of the magnetic signature around a cylindrical cavity of circular cross section with the degree of thermoelectric anisotropy. In this simulation we assumed that the heat flow is directed along the principal direction of the host $\left(\theta=0^{\circ}\right)$ and kept the anisotropy of the electrical and thermal conductivities constant at relatively small levels of $\sigma_{22} / \sigma_{11}=0.98$ and $\kappa_{22} / \kappa_{11}=0.99$, respectively. Generally, the magnetic signature vanishes when the anisotropy of the host vanishes. However, the magnetic signature can also vanish in anisotropic hosts when the relative anisotropy of the three relevant physical properties is such that they cancel each other and their combined effect diminishes [13]. 

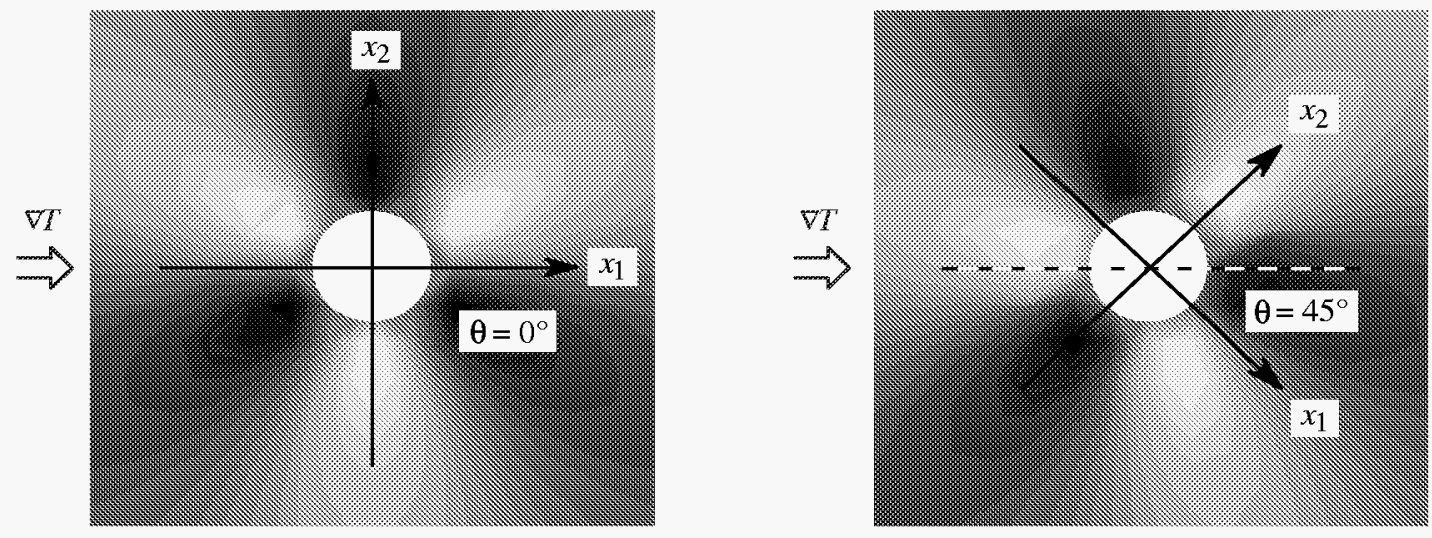

Figure 22 The spatial distribution of the thermoelectric magnetic field around a cylindrical hole of circular cross section for two different orientation of the heat flow with respect to the principal directions of the anisotropic host.

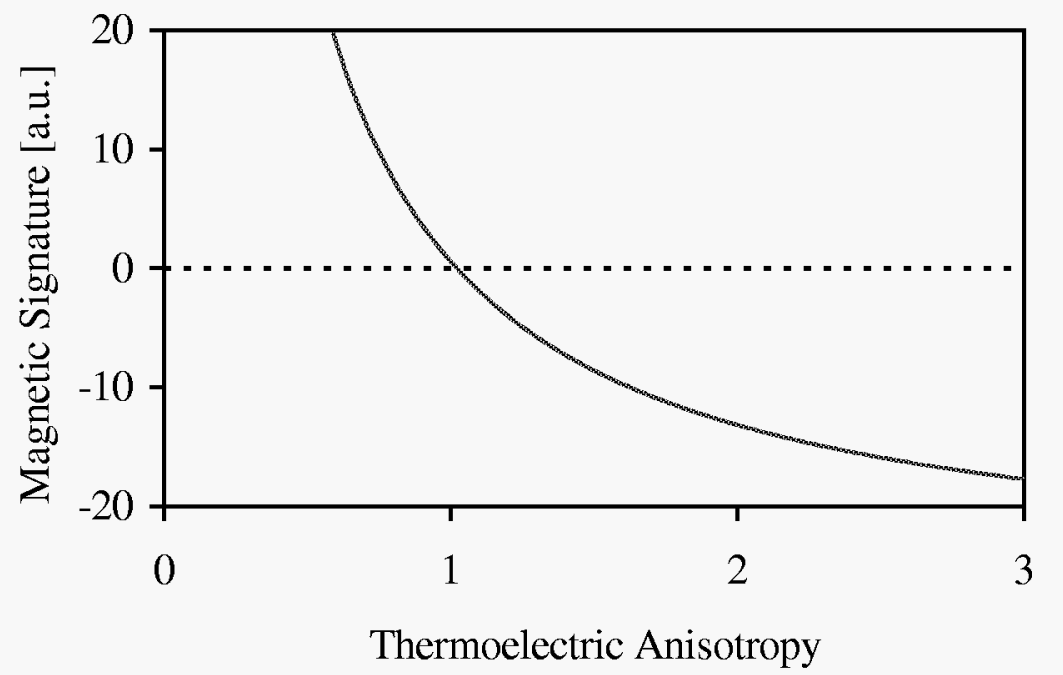

Figure 23 The variation of the magnetic signature around a cylindrical cavity of circular cross section with the degree of thermoelectric anisotropy for heat flow along the principal direction of the host material $\left(\theta=0^{\circ}\right)$.

Although the anisotropic signature of geometric origin clearly interferes with any additional thermoelectric signatures that might have been produced by local variations in the physical properties of the material, it could also produce a useful mechanism for the detection of plastic deformation that produces both crystallographic and morphological texture in the surrounding material. It should be also mentioned that the same analytical model can be also 
used to predict the thermoelectric signatures of embedded isotropic inclusions in anisotropic hosts. Generally, the produced contrast is a combination of the thermoelectric power difference between the inclusion and the host on one side and the anisotropy of the host on the other side. The previously discussed example of a cavity represents only the most extreme case when the contrast is entirely due to the second effect, i.e., no thermoelectric signal would be produced if the host were isotropic. Another case of considerable importance is that of a weak local inhomogeneity caused by heat exposure, cold work, fretting, or other service related damages, when the thermoelectric power difference between the inclusion and the host is relatively small so that the anisotropic contrast is comparable with the inhomogeneous one. In these cases, a better understanding of the contrasts mechanisms is necessary to optimize the detection sensitivity of the thermoelectric inspection method. For example, we have found that dangerous hard-alpha inclusions in titanium alloys can be best detected by repeating the inspection with different heating/cooling directions and averaging the measured signatures. This simple procedure effectively eliminates the anisotropic component of the thermoelectric contrast and greatly enhances the detectability of these brittle, nitrogen-enriched inclusions (see Chapter X).

\section{Thermoelectric signature produced by material inhomogeneity}

The detectability of small and/or weak imperfections is ultimately limited not only by the intrinsic anisotropy of the material, but also by its inhomogeneity. Various factors, such as hardening, plastic deformation, residual stresses, texture, chemical composition, etc., can result in perceivable thermoelectric inhomogeneity of a material. As a first step to study this phenomenon, we investigated the spurious magnetic signature produced by one of the simplest types of macroscopic inhomogeneity, namely when the material properties exhibit a linear spatial variation in the cross-section of an axially heated slender rectangular bar.

Of course, real specimens often exhibit both anisotropy and inhomogeneity, therefore the measured magnetic signature is due to a combination of both effects. Whether the actual signature is dominated by anisotropy or inhomogeneity of the specimen can be simply established by comparing the signatures recorded after rotating the specimen around its principal (length, width, and thickness) axes. Since anisotropic properties are invariant for $180^{\circ}$-rotations, the actual source of the magnetic signature can be always established by repeated measurements 
at different orientations of the bar. In many cases we found that the shape of the background signature essentially remained the same but flipped its sign when the specimen was rotated by $180^{\circ}$ around the direction of heat propagation, which clearly indicates that the observed signal cannot originate from the anisotropy of the specimen unless it is also inhomogeneous. We will show that this rotational symmetry can be exploited not only to separate the effects of anisotropy from those of inhomogeneity, but also to further separate the two principal inhomogeneity components in the thickness- and width-directions from each other.

\section{VII.1 Analytical predictions}

We have already emphasized that, regardless of the size, shape, and material properties of a homogeneous isotropic specimen, no thermoelectric current will be generated by any type of heating or cooling. However, in the presence of material inhomogeneity the thermoelectric current does not necessarily vanish in the specimen. As an example, let us consider a slender bar of rectangular cross section with length $\ell$ much larger than its two other dimensions. The bar is aligned with the $z$-direction of a Cartesian coordinate system $(x, y, z)$ as illustrated in Fig. 24.

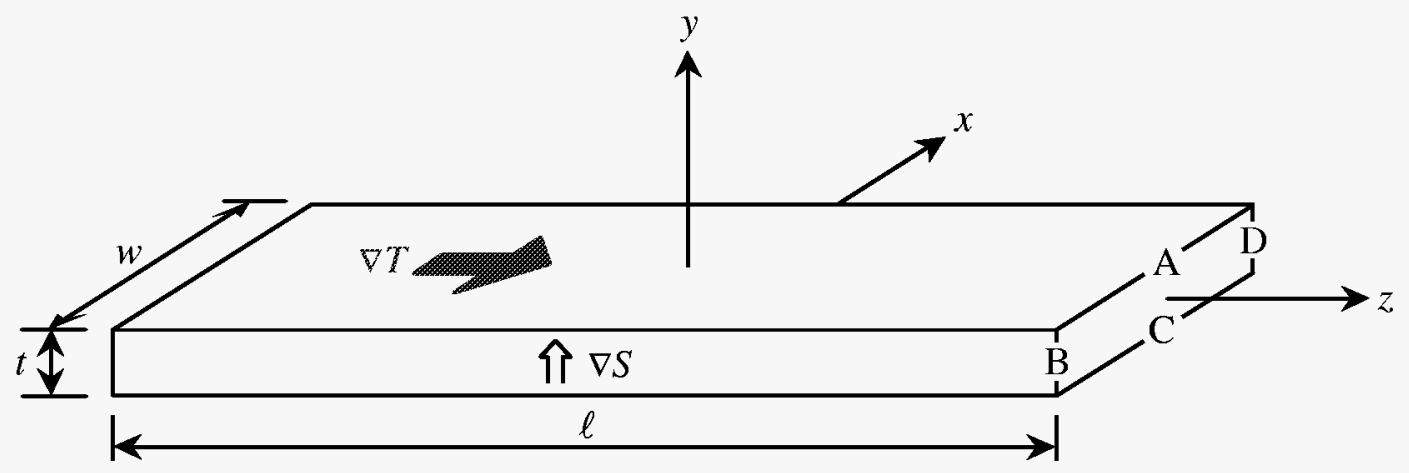

Figure 24 A schematic diagram of the rectangular bar and the Cartesian coordinate system used in the analytical calculations.

In the simplest first-order approximation of inhomogeneity, the spatial dependence of the material properties can be assumed to follow linear profiles

$$
\kappa=\kappa_{0}(1+a y), \sigma=\sigma_{0}(1+b y) \text {, and } S=S_{0}(1+c y) \text {. }
$$


Here, the subscripts 0 refer to the average values of the material properties, while $a, b$ and $c$ are property gradients characterizing the material inhomogeneity. In the case under consideration, material variations along the length of the bar can be neglected partly because the technological effects responsible for the development of linear inhomogeneity (e.g., cold work, residual stress, etc.) do not cause significant axial variations in the material properties and partly because only material variations normal to the direction of heat propagation cause thermoelectric currents. In order to further simplify our analytical model, we assumed property variations along the $y$-direction only. Later, we will present experimental evidence that lateral variations in both thickness- and width-directions of the bar contribute to the observed magnetic signatures. However, this more general two-dimensional inhomogeneity can be easily handled by the onedimensional model using superposition.

The bar is subjected to a uniform axial heat flux $h_{0}$ along the $z$-axis while its sides at $x= \pm w / 2$ and $y= \pm t / 2$ are assumed to be insulated, i.e., we assume that the heat conducted through the metallic bar is much larger than the heat lost by convection and radiation to the surrounding environment. These assumptions reduce the thermoelectric problem into a twodimensional one in the $y, z$ plane. Imposing the one-dimensional spatial distribution of Eq. (44) on the field equations given by $\nabla^{2} T=0$ and $\nabla^{2} \Phi=0$ yields the following coupled governing equations

$$
\begin{aligned}
& \kappa\left[\frac{\partial^{2} T}{\partial y^{2}}+\frac{\partial^{2} T}{\partial z^{2}}\right]+\frac{\partial \kappa}{\partial y} \frac{\partial T}{\partial y}=0 \\
& \sigma S\left[\frac{\partial^{2} T}{\partial y^{2}}+\frac{\partial^{2} T}{\partial z^{2}}\right]+\sigma\left[\frac{\partial^{2} \Phi}{\partial y^{2}}+\frac{\partial^{2} \Phi}{\partial z^{2}}\right]+\left[S \frac{\partial \sigma}{\partial y}+\sigma \frac{\partial S}{\partial y}\right] \frac{\partial T}{\partial y}+\frac{\partial \sigma}{\partial y} \frac{\partial \Phi}{\partial y}=0
\end{aligned}
$$

where we omitted the contribution of the electric field to the heat flux since it is negligible for the typically very low values of the thermoelectric coupling in metals. The exact analytical solution of these coupled equations for arbitrary boundary conditions is fairly complicated. However, for the slender bar under consideration, the complicated temperature and electrical potential distributions near ends are irrelevant far away from the ends, and $T$ and $\Phi$ will be 
simple linear functions of $z$ only, i.e., $T=T(z)=A z$ and $\Phi=\Phi(z)=A^{*} z$, where $A$ and $A^{*}$ are currently unknown constants. Since the sides of the bar are assumed to be insulated, the total heat flux across any cross section normal to the $z$-axis is conserved and should be equal to the total heat flow $\dot{Q}=h_{0} w t$, where $h_{0}$ denotes the average heat flux density. Now, far away from the ends $\dot{Q}$ may be estimated as

$$
\dot{Q}=-w \frac{d T}{d z} \int_{-t / 2}^{t / 2} \kappa(y) d y=-t w A \kappa_{0}
$$

therefore

$$
T=-\frac{h_{0}}{\kappa_{0}} z=\nabla T z
$$

The net electric current through any normal cross section of the bar should be zero

$$
\int_{-t / 2}^{t / 2} j_{z}(y) d y=-\frac{d \Phi}{d z} \int_{-t / 2}^{t / 2} \sigma(y) d y-\frac{d T}{d z} \int_{-t / 2}^{t / 2} \sigma(y) S(y) d y=0
$$

which yields

$$
\Phi \approx-\nabla T S_{0} z
$$

where we exploited that the relative inhomogeneity is low, i.e., $b c t^{2} \ll 1$. Now, we can substitute equations (48) and (50) into the constitutive relations (1) to obtain the electric current density

$$
j_{z} \approx-\sigma_{0} S_{0} c \nabla T y=j_{0} y
$$

where $j_{0}$ is a constant determined by the material properties and $j_{x}=j_{y}=0$. In Eq. (51), we exploited again that the relative inhomogeneity is low.

Generally, the magnetic field produced by the thermoelectric currents can be calculated using the Biot-Savart law 


$$
\mathrm{H}(\mathrm{x})=\int_{-\ell / 2}^{\ell / 2} \int_{-t / 2}^{t / 2} \int_{-w / 2}^{w / 2} \frac{\mathrm{j}(\mathrm{X}) \times(\mathrm{x}-\mathrm{X})}{4 \pi|\mathrm{x}-\mathrm{X}|^{3}} d X d Y d Z
$$

where $\mathrm{x}$ and $\mathrm{X}$ are coordinate vectors of the point of observation and the differential volume of the bar, respectively. Far away from the ends of the long bar neither the electric current density nor the associated magnetic field depends on $z$, therefore, without loss of generality, we can evaluate the integral at $z=0$. Performing the cross product in Eq. (52) and integration along the length of the bar yields

$$
H_{x}(x, y)=-\frac{j_{0}}{2 \pi} \int_{-t / 2}^{t / 2} \int_{-w / 2}^{w / 2} \frac{Y(y-Y)}{(x-X)^{2}+(y-Y)^{2}} d X d Y
$$

and

$$
H_{y}(x, y)=\frac{j_{0}}{2 \pi} \int_{-t / 2}^{t / 2} \int_{-w / 2}^{w / 2} \frac{Y(x-X)}{(x-X)^{2}+(y-Y)^{2}} d X d Y
$$

Integration with respect to $X$ reduces the integrals to the following one-dimensional forms

$$
\begin{aligned}
& H_{x}(x, y)=-\frac{j_{0}}{2 \pi} \int_{-t / 2}^{t / 2} Y\left\{\tan ^{-1}\left[\frac{x+w / 2}{y-Y}\right]-\tan ^{-1}\left[\frac{x-w / 2}{y-Y}\right]\right\} d Y \\
& H_{y}(x, y)=\frac{j_{0}}{4 \pi} \int_{-t / 2}^{t / 2} Y \ln \left[\frac{(x+w / 2)^{2}+(y-Y)^{2}}{(x-w / 2)^{2}+(y-Y)^{2}}\right] d Y
\end{aligned}
$$

Finally, the integrals in Eq. (14) can be solved as follows

$$
\begin{aligned}
& H_{x}(x, y)=H_{0}\left[\left.F_{x}(x+w / 2, y, Y)\right|_{-t / 2} ^{t / 2}-\left.F_{x}(x-w / 2, y, Y)\right|_{-t / 2} ^{t / 2}\right] \\
& H_{y}(x, y)=H_{0}\left[\left.F_{y}(x+w / 2, y, Y)\right|_{-t / 2} ^{t / 2}-\left.F_{y}(x-w / 2, y, Y)\right|_{-t / 2} ^{t / 2}\right]
\end{aligned}
$$


where, after some algebraic simplifications,

$$
\begin{aligned}
& F_{x}(\xi, y, Y)=\xi y \ln \left[1+\left(\frac{y-Y}{\xi}\right)^{2}\right]+\xi Y+\left(Y^{2}-y^{2}+\xi^{2}\right) \tan ^{-1}\left[\frac{y-Y}{\xi}\right], \\
& F_{y}(\xi, y, Y)=\frac{Y^{2}-y^{2}+\xi^{2}}{2} \ln \left[1+\left(\frac{y-Y}{\xi}\right)^{2}\right]-y Y-2 \xi y \tan ^{-1}\left[\frac{y-Y}{\xi}\right], \\
& H_{0}=\frac{j_{0}}{4 \pi}=-\frac{1}{4 \pi} \sigma_{0} S_{0} c \nabla T .
\end{aligned}
$$

\section{VII.2 Experimental arrangement}

In this section, we will describe the experimental setup and procedure we used to verify that the previously introduced analytical model properly predicts the normal and tangential components of the magnetic field produced by thermoelectric currents induced in an inhomogeneous specimen when it is subjected to an external temperature gradient. A Ti-6Al-4V titanium alloy specimen of length $\ell=203 \mathrm{~mm}$, width $w=12.7 \mathrm{~mm}$, and thickness $t=6.35 \mathrm{~mm}$ was cut from a cold-rolled plate. At an arbitrarily chosen orientation, the top, left, bottom, and right sides of the bar were marked as A, B, C, and D, respectively. Then, any other orientation can be easily identified by the order of the edges progressing in a counter-clockwise direction starting from the top. For example, $\mathrm{CBAD}, \mathrm{ADCB}$, and $\mathrm{CDAB}$ correspond to $180^{\circ}$-rotations around the $x, y$, and $z$ axes, respectively, as it is shown in Fig. 25. By exploiting the different odd and even symmetries of the magnetic signatures produced by width- and thickness-inhomogeneity for different rotations, we could easily separate these two contributions to the observed signal.

In order to establish whether the specimen was strongly inhomogeneous and/or anisotropic, we measured the electrical conductivity and absolute thermoelectric power on the four sides of the specimen. First, we used a US-450 (UniWest Corp.) eddy current instrument with a $1.5-\mathrm{mm}$-diameter probe coil at $1 \mathrm{MHz}$ to measure the electrical conductivity. The system was calibrated on reference materials of known electrical conductivity. 100 readings were taken on each side and then averaged to get a representative value. On sides A (0.587 MS) and C 
(0.590 MS) the electrical conductivity was found to be significantly higher than on sides B (0.572 MS) and D (0.570 MS). The $\approx 3 \%$ difference between sides A\&C versus sides B\&D was well above the standard deviation ( $0.003 \mathrm{MS}$ or $0.5 \%)$, which indicates the presence of significant anisotropy in electrical conductivity. On the other hand, the small difference between sides $\mathrm{A}$ and $\mathrm{C}$ or sides $\mathrm{B}$ and $\mathrm{D}$ was less than the standard deviation, therefore we can conclude that conventional eddy current inspection could not unequivocally verify the presence of a significant inhomogeneity in electrical conductivity.

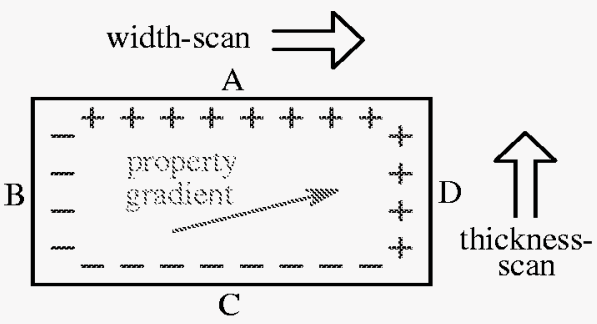

$1 / 4(\mathrm{ABCD}+\mathrm{ADCB}-\mathrm{CDAB}-\mathrm{CBAD})$

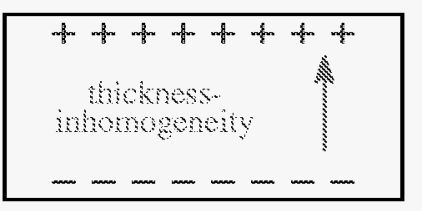

$1 / 4(\mathrm{ABCD}+\mathrm{CBAD}-\mathrm{CDAB}-\mathrm{ADCB})$

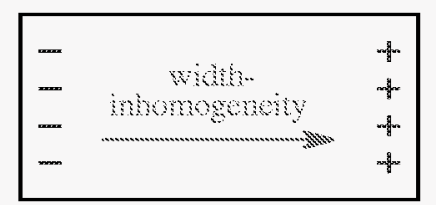

Figure 25 A schematic illustration of how addition and subtraction of different profiles can be used to separate the width- and thickness-inhomogeneity of a specimen exhibiting an arbitrary property gradient.

Second, we measured the thermoelectric power with an ATS-6044 Alloy ThermoSorter (Walker Scientific, Inc.) in an attempt to establish the presence of thermoelectric anisotropy and/or inhomogeneity. Again, the equipment was calibrated on reference materials of known absolute thermoelectric power and 100 readings were taken on each side of the Ti-6Al-4V specimen and then averaged to get a representative value. On sides $\mathrm{A}\left(-5.09 \mu \mathrm{V} /{ }^{\circ} \mathrm{C}\right)$ and $\mathrm{C}(-5.11$ $\mu \mathrm{V} /{ }^{\circ} \mathrm{C}$ ) the thermoelectric power was found to be of significantly higher magnitude than on sides $\mathrm{B}\left(-4.69 \mu \mathrm{V} /{ }^{\circ} \mathrm{C}\right)$ and $\mathrm{D}\left(-4.72 \mu \mathrm{V} /{ }^{\circ} \mathrm{C}\right)$. The $\approx 8 \%$ difference between sides $\mathrm{A} \& \mathrm{C}$ versus sides $\mathrm{B} \& \mathrm{D}$ was again above the standard deviation $\left(0.15 \mu \mathrm{V} /{ }^{\circ} \mathrm{C}\right.$ or $\left.3 \%\right)$, which indicates the presence of a significant anisotropy in thermoelectric power. As before, the small difference between sides $\mathrm{A}$ and $\mathrm{C}$ or sides $\mathrm{B}$ and $\mathrm{D}$ was less than the standard deviation, therefore we can conclude that 
conventional thermoelectric inspection could not unequivocally verify the presence of significant thermoelectric inhomogeneity in the specimen.

In spite of the apparently stronger effect of anisotropy, the thermoelectric background signature of a slender bar could be still dominated by the inhomogeneous contribution because the heat flux is forced to be parallel to the axis of the bar. This could be easily verified by rotating the bar by $180^{\circ}$, upon which the signature should not change at all if it were caused by anisotropy. We found that the signature of the specimen was essentially reversed upon such rotation, therefore it was primarily due to inhomogeneity. We also noticed that the observed change in the signature also depended on how the $180^{\circ}$ rotation was executed. The obvious reason for this is that, in contrast with our simple analytical model, the specimen exhibited property gradients in both width- and thickness-directions.

Figure 26 shows a schematic diagram for the experimental arrangement used to study the different thermoelectric signatures produced by the bar. The fluxgate magnetometer can be polarized either tangential or normal to the top surface in order to measure the $x$ and $y$ components of the magnetic field, respectively. The specimen was mounted on two copper supports that also acted as heat exchangers to facilitate efficient heating and cooling and the whole assembly was mounted on a non-magnetic translation table for scanning. In order to improve the heat transfer, a layer of heat conducting silicone grease compound was applied between the specimen and the copper heat exchangers, which were heated and cooled to temperatures of $85^{\circ} \mathrm{C}$ and $5{ }^{\circ} \mathrm{C}$, respectively. The actual temperature of the Ti-6Al-4V specimen was monitored by thermocouples at two points and the temperature gradient along the center part of the bar was kept at $4.5^{\circ} \mathrm{C} / \mathrm{cm}$ in all measurements.

The magnetic signals were detected by horizontally scanning the specimen at the center of the bar in a direction normal to the heat flux. Figure 26 showed how the normal and tangential magnetic signatures can be recorded when scanning in the "width-direction" of the specimen. Since the translation table can move only horizontally, but not vertically, similar scans in the thickness-direction of the specimen were taken by rotating the specimen $90^{\circ}$ around its axis and lying it on its thinner side. As we described above, in order to separate the "width" and "thickness" components of the inhomogeneity, we have to record the magnetic signature at four specimen orientations. Altogether, sixteen independent signatures can be measured using eight specimen orientations and two sensor polarizations. However, all magnetic signatures were 
recorded with both positive and negative temperature gradient and then subtracted to separate the truly thermoelectric component from spurious contributions of external sources. Therefore, the experimental data to be presented later represents thirty-two measurements..

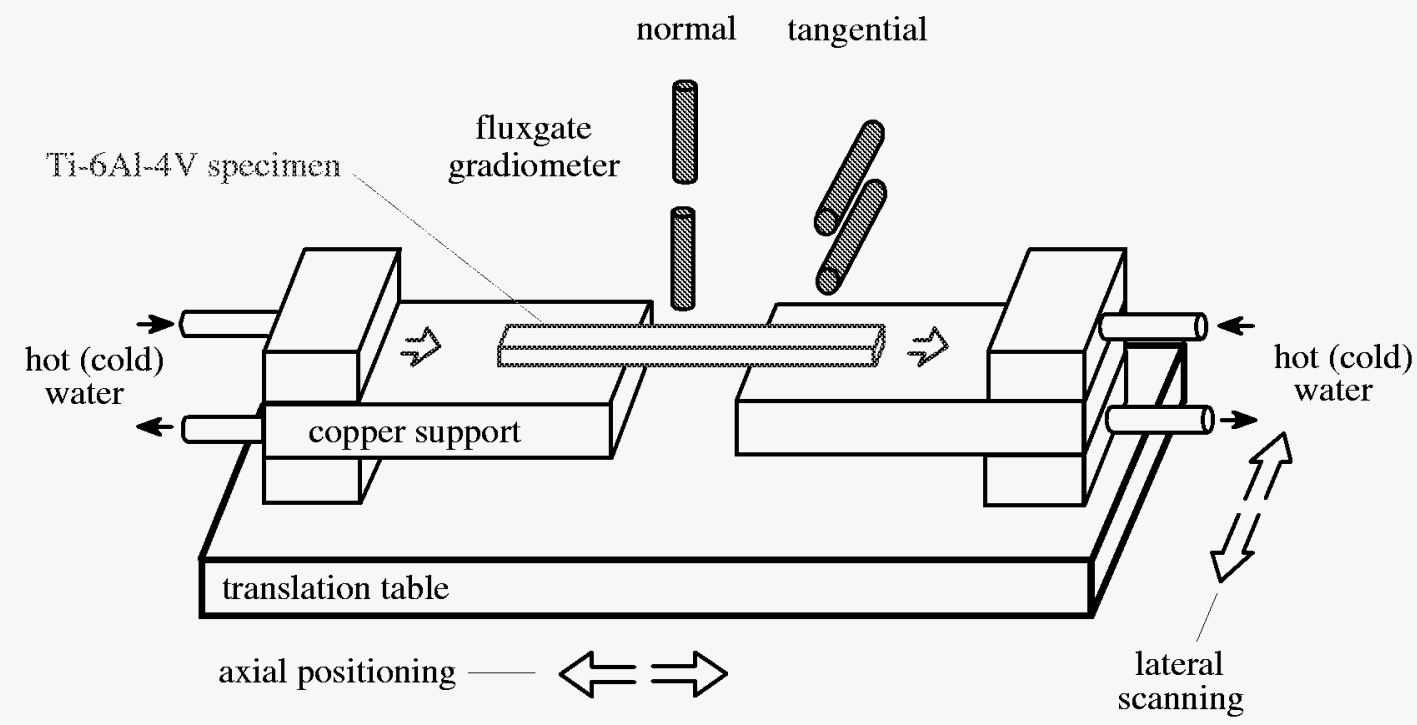

Figure 26 A schematic diagram for the experimental arrangement.

\section{VII.3 Experimental results in Ti-6Al-4V}

In order to compare the measured spatial distribution of the magnetic field due to the assumed inhomogeneity profile, we used the known geometrical dimensions of the specimen. The relevant physical parameters of $\mathrm{Ti}-6 \mathrm{Al}-4 \mathrm{~V}$ that are needed for substitution into our analytical model are $\sigma_{0}=5.7 \times 10^{5} \mathrm{~A} / \mathrm{Vm}$ and $S_{0}=-4.9 \mu \mathrm{V} /{ }^{\circ} \mathrm{C}$. Two free parameters, that measure the inhomogeneity in the width $c_{x}$ and thickness $c_{y}$ directions, were used to find the best fit between the model predictions and the four experimentally determined signatures in each case. The average of the four best fitting values of $c_{x}$ can be considered as the best estimates for the actual degree of inhomogeneity in the width-direction, while the same is true for the average of the four best fitting values of $c_{y}$ and the inhomogeneity in the thickness-direction of the specimen. Though these values cannot be independently verified at this point, the fact that the four values are reasonably close to each other in both cases lends some confidence to the obtained values. 
Figure 27 shows the comparison between the experimental and theoretical thermoelectric signatures for thickness-inhomogeneity. The width-signature at normal polarization (Fig. 27a) corresponds to $H_{y}\left(x, y_{p n}\right)-H_{y}\left(x, y_{s n}\right)$, where $y_{p n}=g_{p n}+t / 2$ and $y_{s n}=g_{s n}+t / 2$ are the constant distances of the primary and secondary fluxgates from the $x$-axis at normal polarization. It was noticed that the relatively sharp peaks on the theoretical curves were always somewhat blurred on the experimental results by the finite size of the active element that reduces the spatial resolution of the measurement. Therefore, the theoretical curves were smoothened by averaging over a distance equal to the size of the fluxgate $( \pm 7.5 \mathrm{~mm}$ for tangential polarization and $\pm 1 \mathrm{~mm}$ for normal polarization) in order to simulate the reduced resolution of the experimental data. With this correction, the agreement between the shapes of the theoretical and experimental signatures is very good. The width-signature at tangential polarization (Fig. 27b) corresponds to $H_{x}\left(x, y_{t p}\right)-H_{x}\left(x, y_{t s}\right)$, where $y_{t p}=g_{t p}+t / 2$ and $y_{t s}=g_{t s}+t / 2$ are the constant distances of the primary and secondary fluxgates from the $x$-axis at tangential polarization. In a similar way, Figs. 27c and 27d show the comparison between the experimental and theoretical thermoelectric signatures for scanning in the thickness-direction. It should be emphasized that the very good qualitative agreement between the experimental and theoretical data shown is partially due to the fact that each curve was individually best fitted by changing the thickness-inhomogeneity parameter $c_{y}$ (these values are listed on each figure). The average of the four $c_{y}$ values was $22.1 \mathrm{~m}^{-1}$, with a standard deviation of $2.7 \mathrm{~m}^{-1}$, which indicates that the accuracy of the thickness-inhomogeneity assessment is $\approx \pm 12 \%$.

Using the symmetry relationships shown in Fig. 25, the width-inhomogeneity of the specimen can be separately evaluated. Figure 28 shows the comparison between the experimental and theoretical thermoelectric signatures caused by width-inhomogeneity of the specimen. The average value of the four $c_{x}$ values was $3.4 \mathrm{~m}^{-1}$, with a standard deviation of $0.4 \mathrm{~m}^{-1}$, which indicates that the accuracy of the width-inhomogeneity assessment is also $\approx \pm$ $12 \%$. Considering the rather crude approximations used in the theoretical calculations to account for the finite size of the sensor elements, the fact that the secondary sensor is partially shielded by the primary sensor, and the experimental errors associated with the measurement, the agreement between the experimental and theoretical thermoelectric magnetic signatures is surprisingly good. 
(a) width-scan, normal polarization

$$
\left(c_{y}=22.1 \mathrm{~m}^{-1}\right)
$$

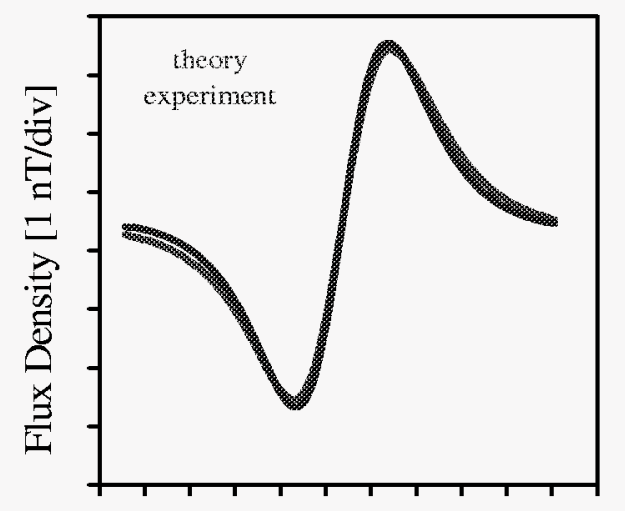

Distance $[10 \mathrm{~mm} / \mathrm{div}]$

(c) thickness-scan, normal polarization

$$
\left(c_{y}=19.1 \mathrm{~m}^{-1}\right)
$$

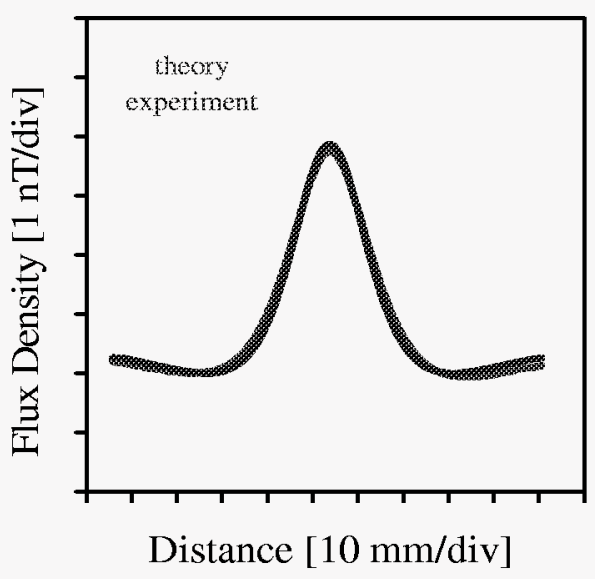

(b) width-scan, tangential polarization

$$
\left(c_{y}=25.8 \mathrm{~m}^{-1}\right)
$$

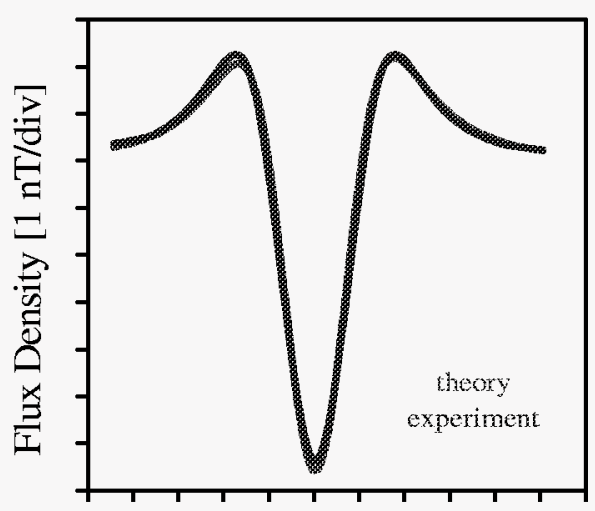

Distance $[10 \mathrm{~mm} / \mathrm{div}]$

(d) thickness-scan, tangential polarization

$$
\left(c_{y}=21.7 \mathrm{~m}^{-1}\right)
$$

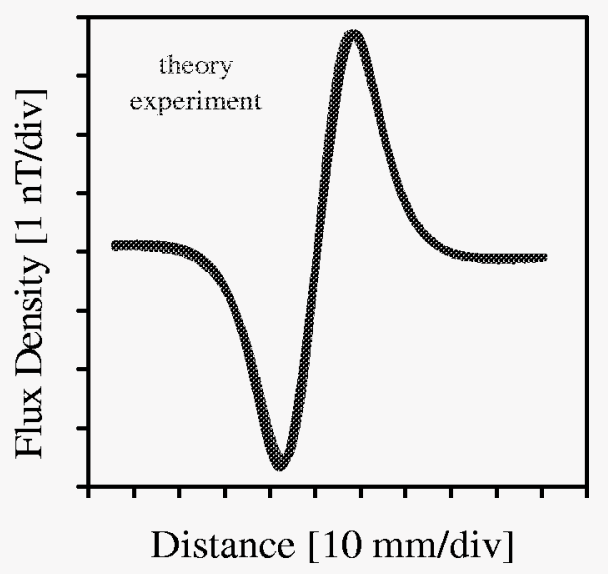

Figure 27 Comparison between the experimental and theoretical thermoelectric signatures for thickness-inhomogeneity in the slightly inhomogeneous Ti-6AL-4V specimen.

These results verify that the observed magnetic signatures are indeed caused by a linear thermoelectric current distribution of the form $j_{z}=j_{0 x} x+j_{0 y} y$ in the slender bar. However, the magnitude of the observed signatures raises serious doubts about the reliability of the previously described contact measurements. The $c_{x^{w}} \approx 0.043$ and $c_{y} t \approx 0.14$ products represent our estimates for the total relative variation of the thermoelectric power through the width and thickness of the slender bar, respectively. These numbers are too large to be reconciled with the 
independent experimental measurements by a conventional thermoelectric alloy sorter. The most probable reason for this lack of consistency is the strong anisotropy of Ti-6Al-4V [23], which preferentially crystallizes in hexagonal symmetry, and its susceptibility to measurement errors by the contact technique [4].

(a) width-scan, normal polarization

$$
\left(c_{x}=2.8 \mathrm{~m}^{-1}\right)
$$

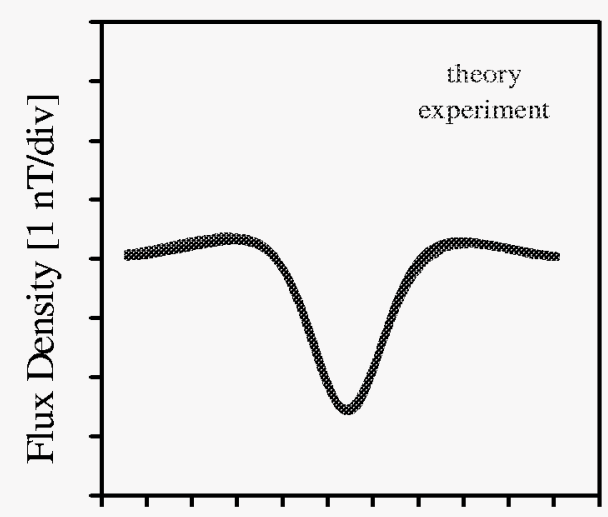

Distance $[10 \mathrm{~mm} / \mathrm{div}]$

(c) thickness-scan, normal polarization

$$
\left(c_{x}=3.4 \mathrm{~m}^{-1}\right)
$$

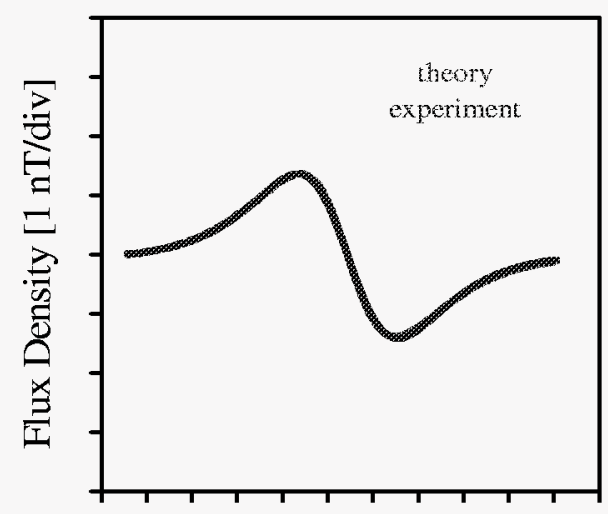

Distance $[10 \mathrm{~mm} / \mathrm{div}]$ (b) width-scan, tangential polarization

$$
\left(c_{x}=3.5 \mathrm{~m}^{-1}\right)
$$

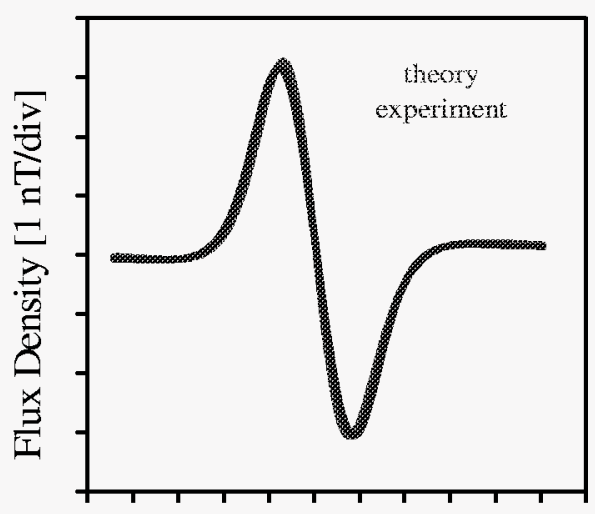

Distance $[10 \mathrm{~mm} / \mathrm{div}]$

(d) thickness-scan, tangential polarization

$$
\left(c_{x}=3.7 \mathrm{~m}^{-1}\right)
$$

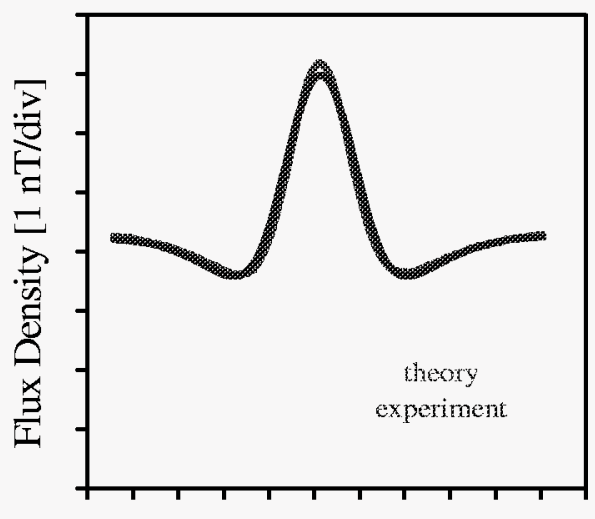

Distance $[10 \mathrm{~mm} / \mathrm{div}]$

Figure 28 Comparison between the experimental and theoretical thermoelectric signatures for width-inhomogeneity in the slightly inhomogeneous Ti-6AL-4V specimen. 


\section{VII.4 Experimental results in brass}

In order to independently verify the validity of our analytical model, we prepared an artificial specimen that exhibits a well-defined inhomogeneity that is large enough to be measured not only by the uniquely sensitive noncontacting method but also by the far less sensitive conventional contact method. For this purpose, we obtained an inhomogeneous sintered specimen made from copper and brass by powder metallurgy. This specimen was $w=7 \mathrm{~mm}$ wide, $t=13.5 \mathrm{~mm}$ thick, and $\ell=162 \mathrm{~mm}$ long with properties varying only in the thickness $(y)$ direction. The composition of this sintered specimen changes from pure copper on one side to brass ( $80 \%$ copper, $20 \%$ zinc) on the other, which blend well at high sintering temperatures. Based on the perceivable change in color from reddish (copper) to yellowish (brass), the transition was fairly smooth and linear. Although we had no means of actually verifying the assumed dependence of the thermoelectric power, the only parameter that is really important in our model, on position throughout the thickness of the specimen, it is reasonable to approximate this variation with a linear function changing from the thermoelectric power of pure copper to that of brass. This seems to work for the electrical conductivity, which can be mapped by scanning the side of the specimen with a small eddy current probe.

Figure 29 shows the eddy current image of the conductivity variation in this artificial copper/brass reference specimen, which was obtained by a small 1.25 -mm-diameter coil at 85 kHz. Like in the thermoelectric measurements, this processed image was constructed by the above described averaging procedure from four raw images taken from the two sides of the specimen. The electric conductivity changed from $\sigma_{\mathrm{Cu}}=79.5 \%$ IACS on the copper side to $\sigma_{\mathrm{Br}}=29.3 \% \mathrm{IACS}$ on the brass side. In our analytical calculations we used the average of these two values, i.e., $\sigma_{0}=54.4 \% \operatorname{IACS}\left(3.25 \times 10^{7} \mathrm{~A} / \mathrm{Vm}\right)$. We have also had two reference pieces made from pure copper and pure brass powder under the same conditions (sintering temperature and time) to measure their respective thermoelectric power and estimated the thermoelectric power of the inhomogeneous specimen by linear regression. The average difference in thermoelectric power between pure copper and brass was found to be $S_{\mathrm{Cu}}-S_{\mathrm{Br}}=\Delta S=0.5 \mu \mathrm{V} /{ }^{\circ} \mathrm{C}$. In order to calculate the magnetic signatures predicted by our analytical model, $\Delta S=S_{0} c t$ must be substituted into Eq. (56c). 

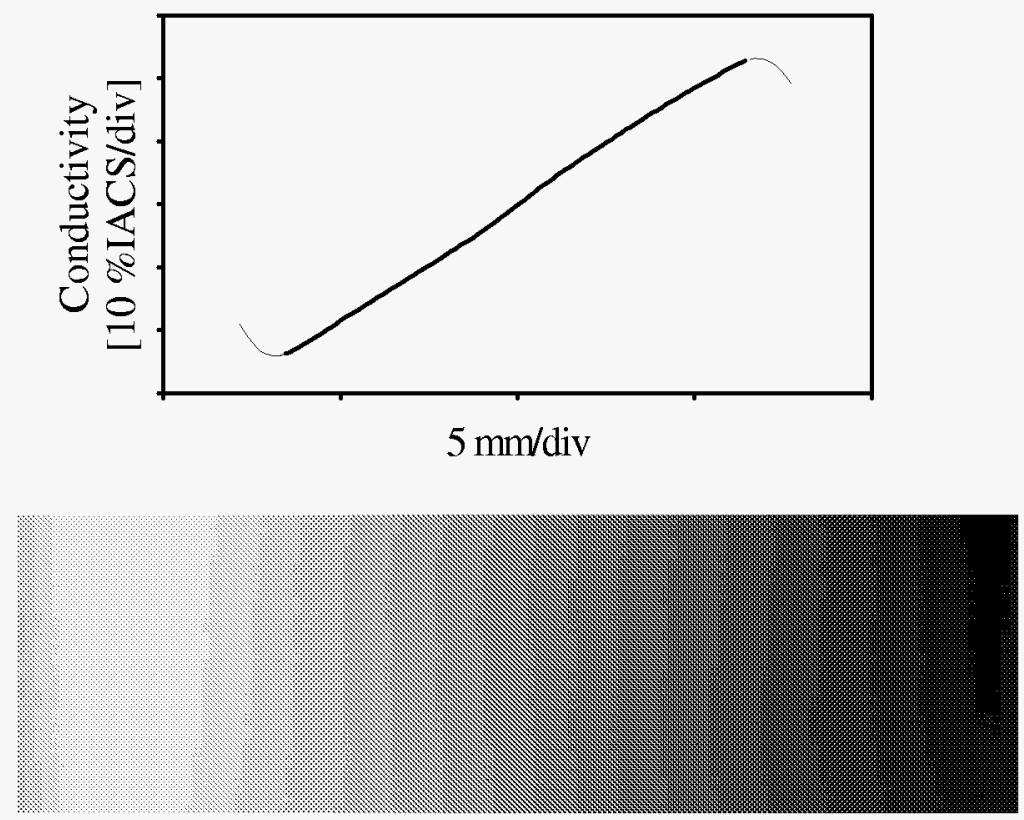

Figure 29 Eddy current image of the conductivity variation in the artificial copper/brass reference specimen.

Needless to say this high-conductivity specimen yielded roughly two orders of magnitude stronger magnetic signals than the typical Ti-6Al-4V bar used in our previous experiment. Figure 30 shows the comparison between the experimental and theoretical thermoelectric signatures for thickness-inhomogeneity. The temperature gradient imposed on the specimen during these measurements was $\nabla T=3.6^{\circ} \mathrm{C} / \mathrm{cm}$. Again, the four signatures measured for the thicknessinhomogeneity of the specimen were individually best fitted by the predictions of our analytical model and the resulting thermoelectric contrast is listed for each case. Based on these four measurements the average thermoelectric contrast is $\Delta S \approx 0.57 \mu \mathrm{V} /{ }^{\circ} \mathrm{C}$ with a standard deviation of $0.08 \mu \mathrm{V} /{ }^{\circ} \mathrm{C}$, which is in very good agreement with the $\Delta S \approx 0.5 \mu \mathrm{V} /{ }^{\circ} \mathrm{C}$ value independently measured by contact means. The slight overestimation seems to be mainly caused by the data obtained at tangential polarization when the effect of the sensor size and the shielding effect of the primary sensor on the signal measured by the secondary sensor have a more significant effect on the measured overall signature. Because of these effects, which would be very difficult to include in the analytical model, it appears that all the numbers obtained at tangential polarization are a little higher than those obtained at normal polarization. 
(a) width-scan, normal polarization

$$
\left(\Delta S=0.45 \mu \mathrm{V} /{ }^{\circ} \mathrm{C}\right)
$$

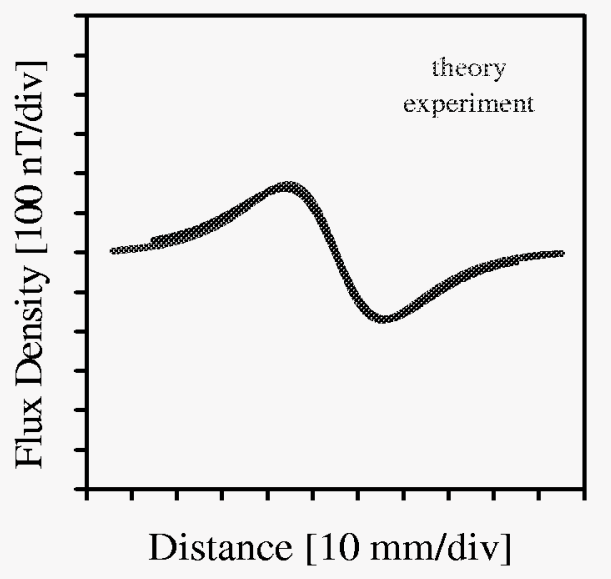

(c) thickness-scan, normal polarization

$$
\left(\Delta S=0.53 \mu \mathrm{V} /{ }^{\circ} \mathrm{C}\right)
$$

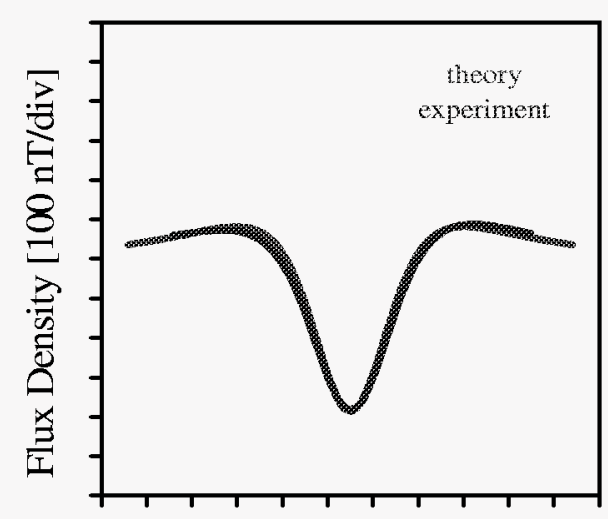

Distance $[10 \mathrm{~mm} / \mathrm{div}]$ (b) width-scan, tangential polarization

$$
\left(\Delta S=0.70 \mu \mathrm{V} /{ }^{\circ} \mathrm{C}\right)
$$

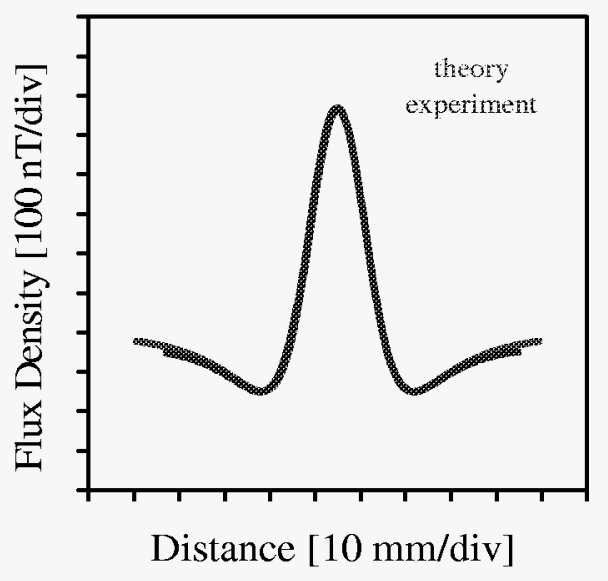

(d) thickness-scan, tangential polarization

$$
\left(\Delta S=0.64 \mu \mathrm{V} /{ }^{\circ} \mathrm{C}\right)
$$

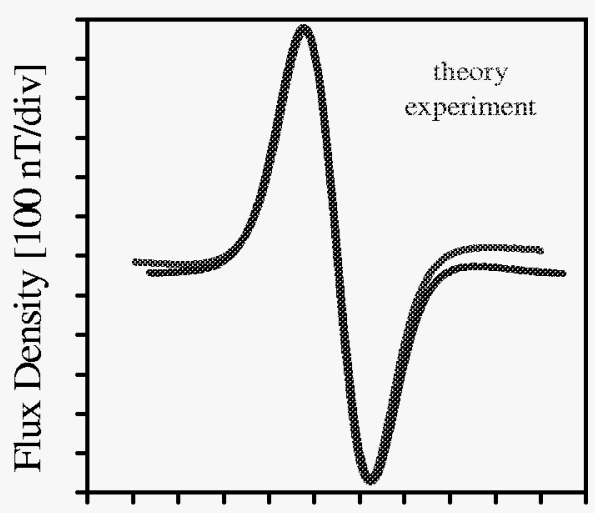

Distance $[10 \mathrm{~mm} / \mathrm{div}]$

Figure 30 Comparison between the experimental and theoretical thermoelectric signatures for thickness-inhomogeneity in the strongly inhomogeneous sintered copper/brass specimen.

The measured width-inhomogeneity (not shown) was found to be very small, roughly twenty times lower than the thickness-inhomogeneity. Of course, it should be really zero since the variation is limited to the thickness direction only, but the modest accuracy of the additions/subtractions used to separate these two effects based on their respective symmetry sets a limit on the rejection ratio. In short, our measurements on the artificial reference specimen 
have verified our analytical model, though at a level much higher than those encountered in real specimens. Unfortunately, this is unavoidable because the magnetic technique is orders of magnitude more sensitive than the conventional contact thermoelectric testers currently available for the limited purposes of alloys sorting.

In this section we presented an analytical method for calculating the normal and tangential magnetic fields produced by thermoelectric currents due to the presence of weak inhomogeneity in a slender rectangular bar under axial heating and cooling. Experimental results from a Ti-6Al-4V titanium-alloy bar were shown to be in very good qualitative agreement with the predictions of the analytical model. These results clearly indicate that the observed background signatures on the four sides of the specimen can be attributed to a linear thermoelectric current distribution in the cross section of the slender bar. However, the high magnitude of the observed signatures made it improbable that the actual source of these thermoelectric currents is simply a minor inhomogeneity of the material. Therefore additional measurements were made on an artificial copper/brass sintered specimen that exhibited a very large thermoelectric contrast which could be measured by conventional contact thermoelectric methods. These measurements were found to be in very good quantitative agreement with our theoretical predictions and fully verified our analytical model. As for the unexpectedly high signature observed in the Ti-6Al-4V specimen, further efforts are needed to better understand the underlying physical phenomenon and to clarify the relationship between the strength of the thermoelectric signature and the very complex microstructural features of the material. However, even without positively identifying the physical source of the thermoelectric signature, we can conclude that in such specimens the best flaw detectability can be achieved by rotating the specimen and measuring both normal and tangential magnetic signatures so that even arbitrary two-dimensional inhomogeneity could be averaged out. These results can be used to optimize thermoelectric inspection procedures for flaw detection as well as to develop techniques capable of quantitatively assessing the thermoelectric inhomogeneity of metals.

\section{Thermoelectric signature produced by applied stresses}

In an effort to develop the experimental tools needed to quantitatively assess the stressdependence of the thermoelectric power, $\mathrm{C} 1100$ pure copper was considered to study the effects 
of the elastic strain in the absence of the plastic deformation in the simple case of a plate in bending as shown in Fig. 31. Because of the stress-dependence of the thermoelectric power of the material, depending on whether the slope of the stress-dependence is positive or negative, a thermoelectric current loop will form running in either clockwise or counter-clockwise direction, thereby generating a positive or negative magnetic field.

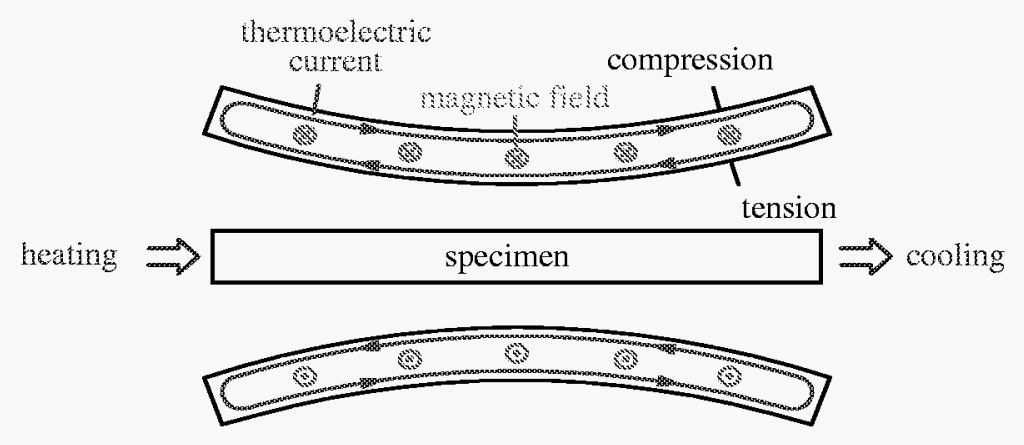

Figure 31 A schematic diagram of the magnetic field produced due to linear variation of stress along the beam when unidirectional temperature gradient is applied.

In order to study the stress-dependence of the thermoelectric signature in a bar in bending, a three-point bending machine was built using nonmagnetic polycarbonate material. The supports were designed to have a relatively high moment of inertia to eliminate deflection in the direction of the applied load as shown in Fig. 32. Fine displacement at the middle of the span was achieved through using a fine threaded screw. A pure copper bar of $355.6 \mathrm{~mm} \times 38.1 \mathrm{~mm} \times$ $3.175 \mathrm{~mm}$ dimensions was prepared. A thin bar was considered to increase the linear variation of stress across the material and to reduce its moment of inertia against bending. Both ends of the copper bar were perforated by a series of holes and equipped with sealed heat exchangers to facilitate efficient heating and cooling and then mounted on a nonmagnetic translation table for scanning. Since sufficiently fast alternating heating could not be implemented because of the inherently sluggish thermal response of the specimen, all measurements were done under a steady-state thermal condition that was achieved in a few minutes after starting the heating and cooling. 


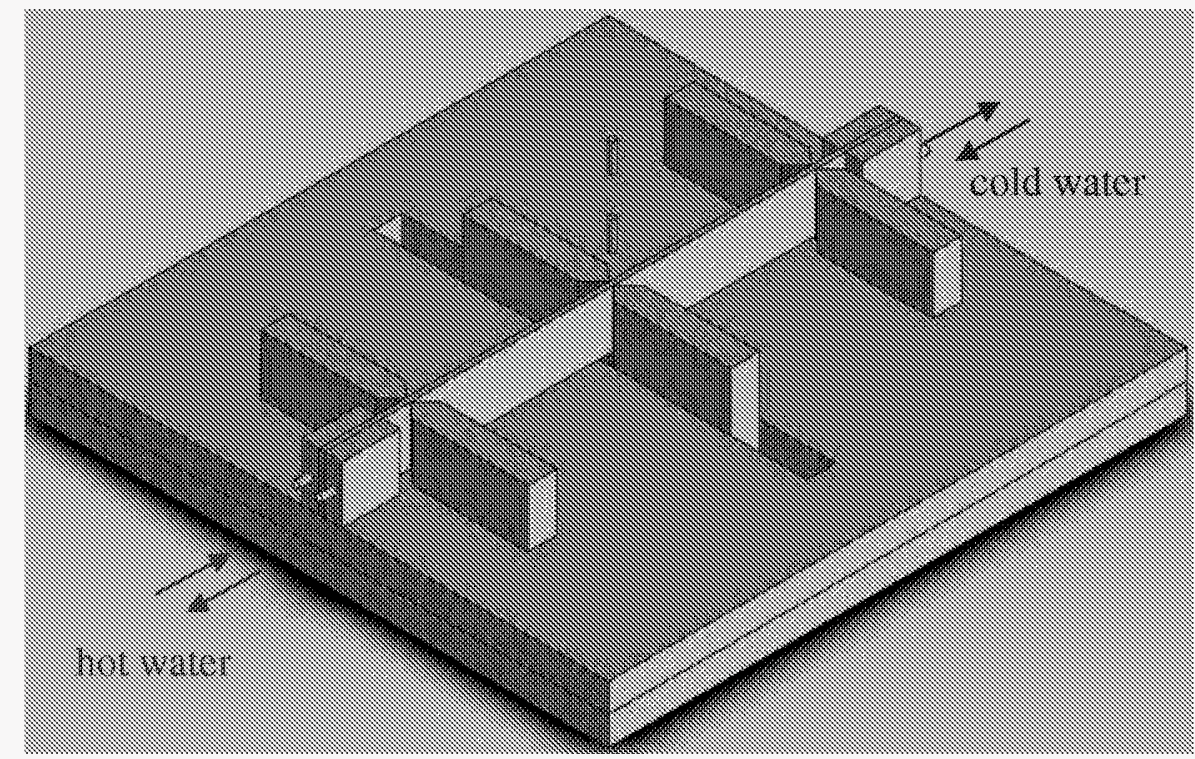

Figure 32 The lay out of the experimental setup to study the effect of the stress linear variation on the thermoelectric magnetic signal.

In order to reach the high sensitivity required in these measurements, non-stationary extraneous magnetic fields had to be rejected. Most of the ac magnetic interference occurs at the $60 \mathrm{~Hz}$ mains frequency and its upper harmonics. These signals were effectively eliminated by a very sharp (six-pole) low-pass filter of $3 \mathrm{~Hz}$ cutoff frequency. A high-pass filter of $0.3 \mathrm{~Hz}$ was applied to eliminate the small variation in the earth magnetic field, which is around $50 \mu \mathrm{T}$. The signals to be detected are generated in the close vicinity of the magnetometer, therefore they significantly vary from point to point, i.e., they exhibit strong gradients. Extraneous signals typically originate at larger distances from the magnetometer, therefore they smoothly vary from point to point, i.e., they exhibit relatively small gradients. In order to exploit this difference, we used a pair of detectors in a gradiometric arrangement. The primary sensor closer to the specimen (1-mil lift-off) picks up a much stronger signal from the inclusion than the secondary sensor further away, while the two sensors exhibit essentially the same sensitivity for sources at large distances. The baseline distance was chosen to be $28.6 \mathrm{~mm}$ in our case (generally, baseline optimization depends on the spatial distribution of the magnetic field to be measured). Further reduction of the baseline distance would improve the rejection of extraneous signals but would also reduce the sensitivity to the thermoelectric signals to be detected. 


\section{VIII.1 Experimental result}

After applying the temperature gradient along the pure copper specimen, the reference thermoelectric magnetic signal generated due to the texture variation along the specimen was measured. This signature is shown in Fig. 33 (a) and (b) for positive and negative temperature gradients, respectively. Then, elastic bending was applied on the specimen using the three-point bending machine to put the front side of the specimen either under tension or compression. The linear variation of the applied stress across the material produced an additional thermoelectric current, which is indicated by a change in the thermoelectric signature of the specimen. The measured signatures under tension and compression of the front surface at $40 \%$ stress ratio with respect to yield stress are also shown in Fig. 33 (a) and (b). These signals represent the linear superposition of the original texture related magnetic signal and the stress related magnetic signal.

To better separate the effect of applied stress from the intrinsic texture of the material, the thermoelectric magnetic signatures measured after applying tension or compression on the front surface were subtracted from the texture related baseline signature, as shown in Fig. 34 (a) and (b), respectively. The maximum variation in the thermoelectric magnetic signature can be produced by comparing the generated signals when one face is under tension and then when it is under compression, as shown in Fig. 34 (a) and (b) for both temperature gradient directions. If these signals were entirely of thermoelectric origin, the sum of these signals generated under positive and negative temperature gradients would equal to zero. The net stress related thermoelectric magnetic signature can be best represented by half of the difference between the raw signatures received under positive and negative temperature gradients as shown in Fig. 35.

Figure 36 demonstrates how increasing the stress level (as measured by the stress ratio which is defined as the peak stress divided by the yield strength of the material) increases the strength of the thermoelectric magnetic signature. To further illustrate the effect of increasing the stress ratio on the net stress related thermoelectric magnetic signature, the peak-to-peak magnitude of the magnetic signature was measured. As shown in Fig. 37, this magnitude increases linearly as the stress ratio increases in the elastic region. 
a) negative temperature gradient

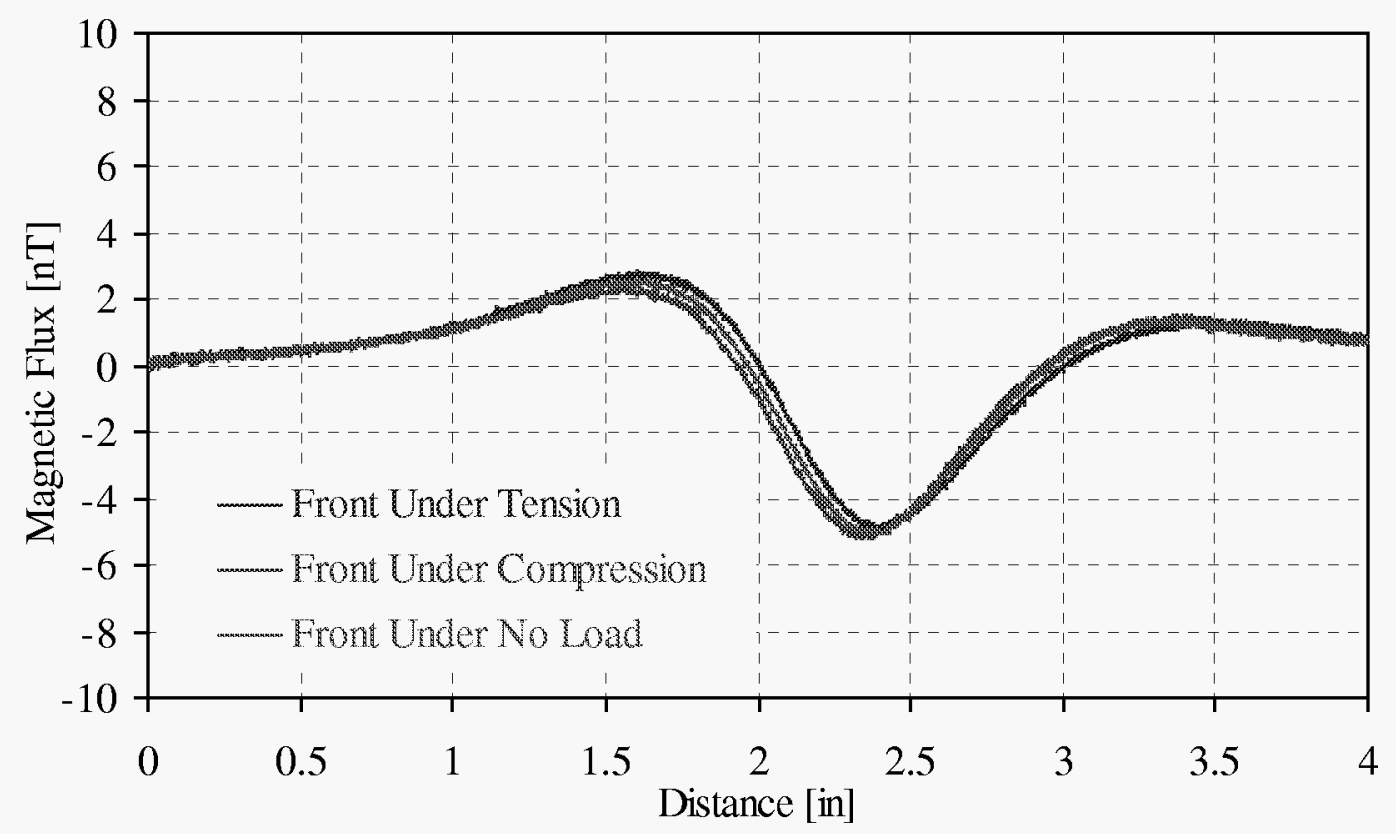

b) positive temperature gradient

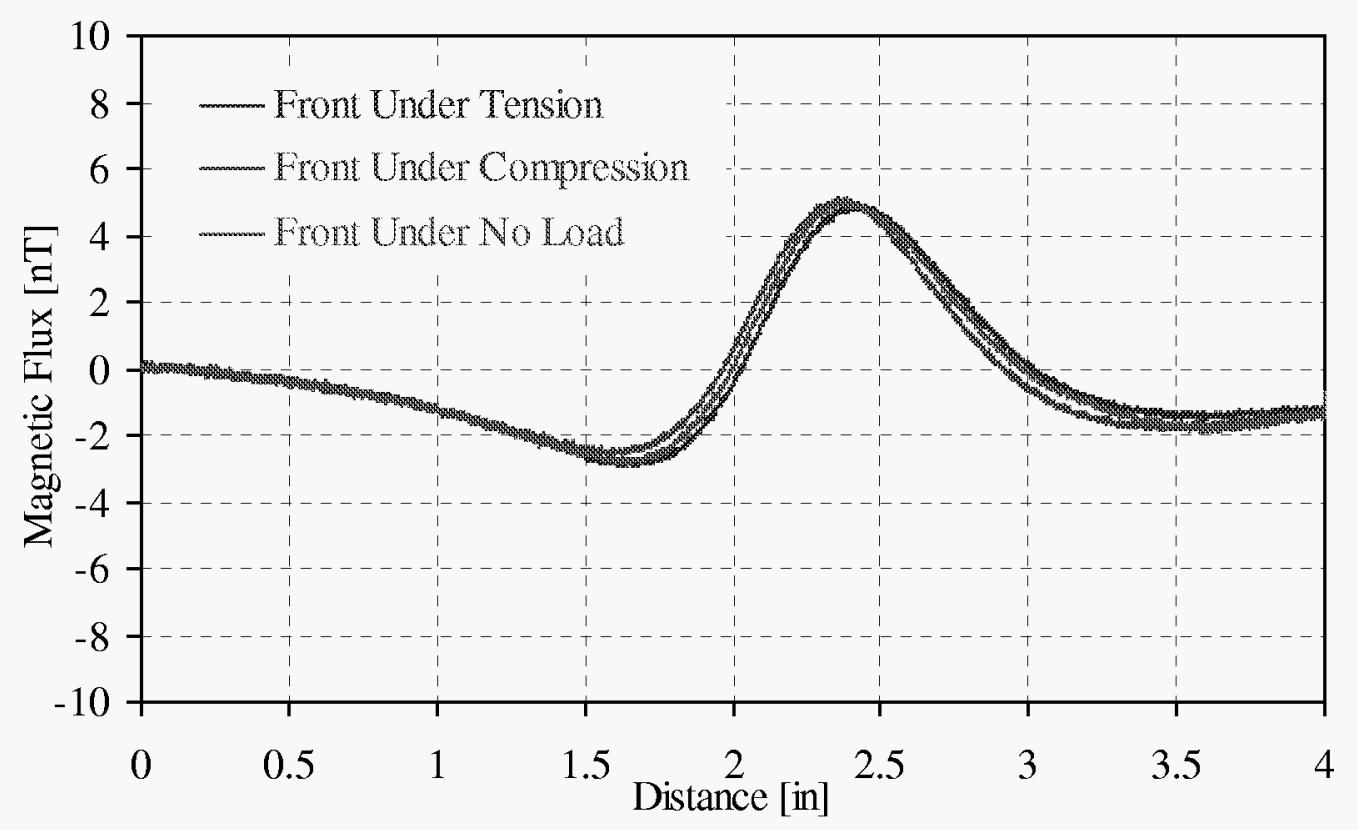

Figure 33 The measured thermoelectric signature under three different loading conditions (40\% stress ratio) with (a) positive and (b) negative temperature gradients. 


\section{a) negative temperature gradient}

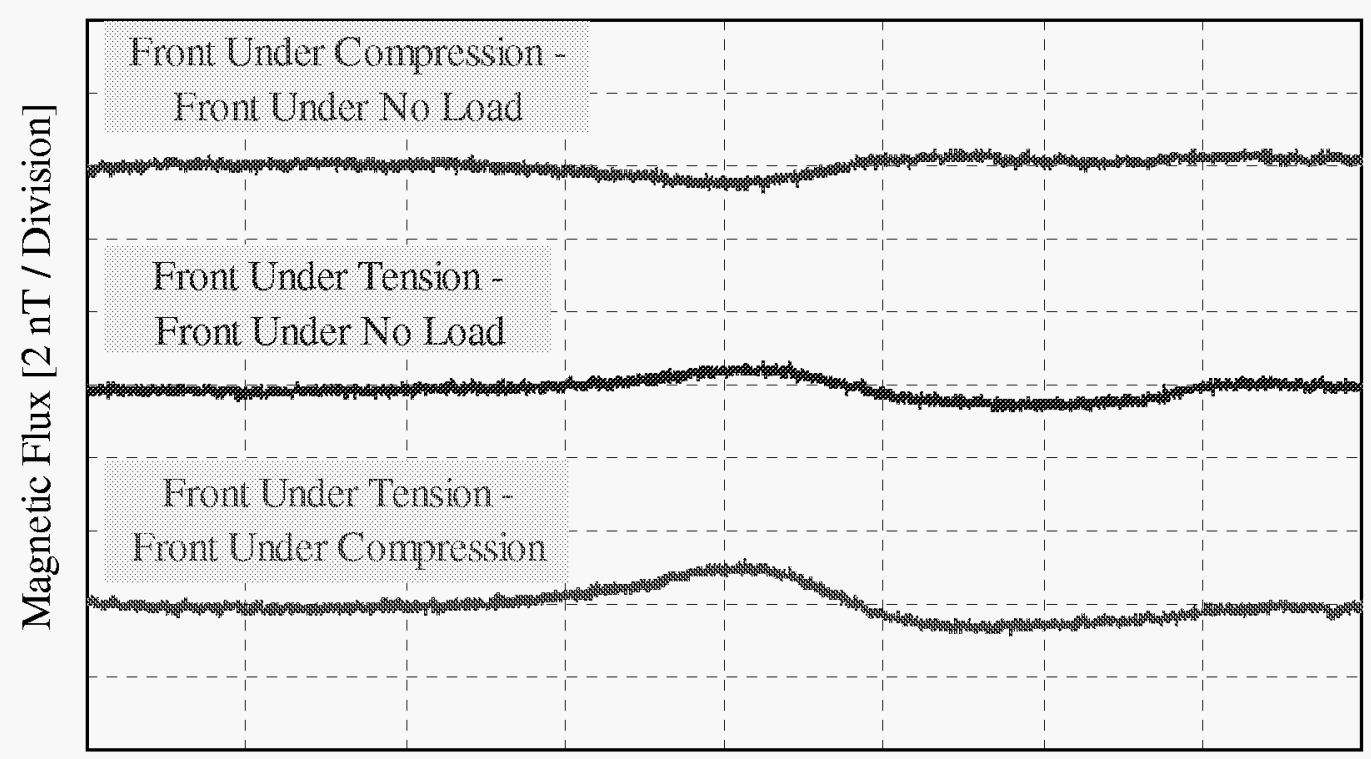

Distance [0.5 in / Division]

b) positive temperature gradient

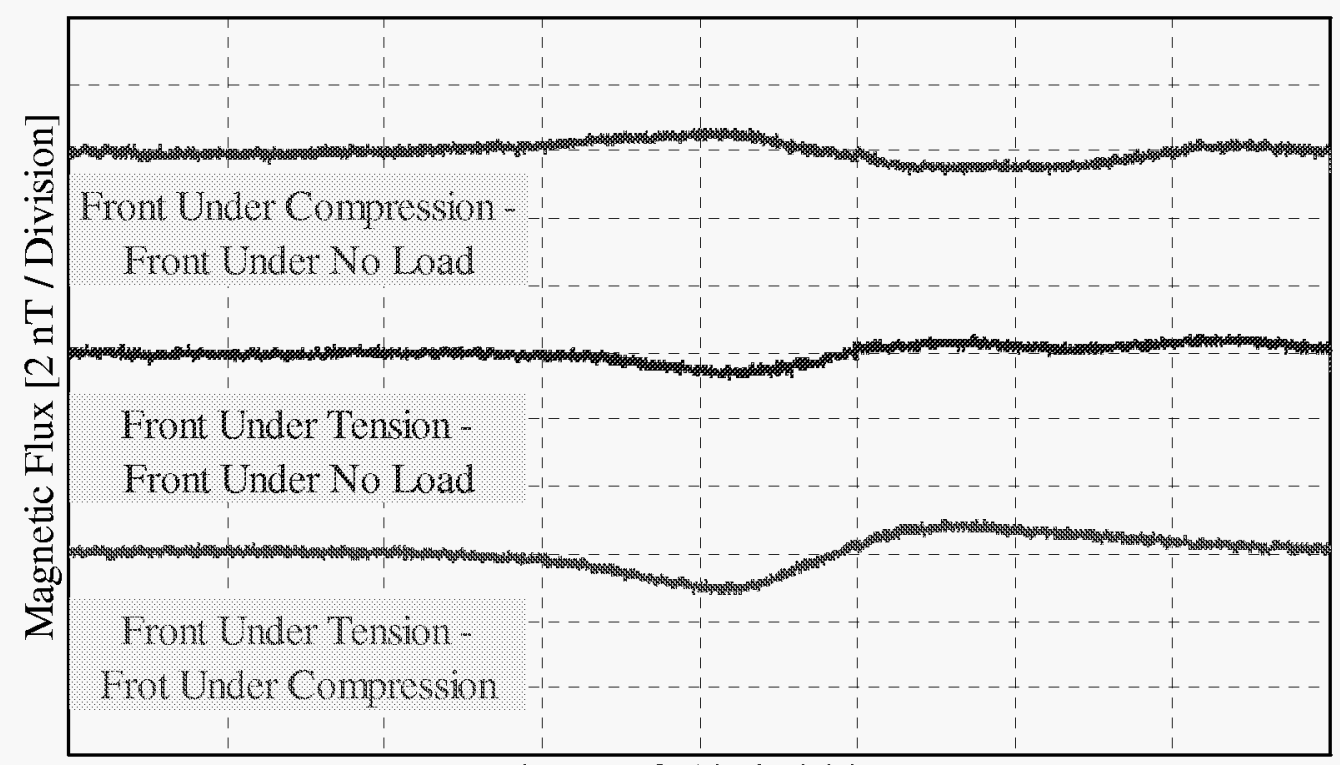

Distance [0.5 in / Division]

Figure 34 The stress related thermoelectric magnetic signal produced under $40 \%$ stress ratio. 




Figure 35 The net stress related thermoelectric magnetic signal produced under positive and negative temperature gradients.

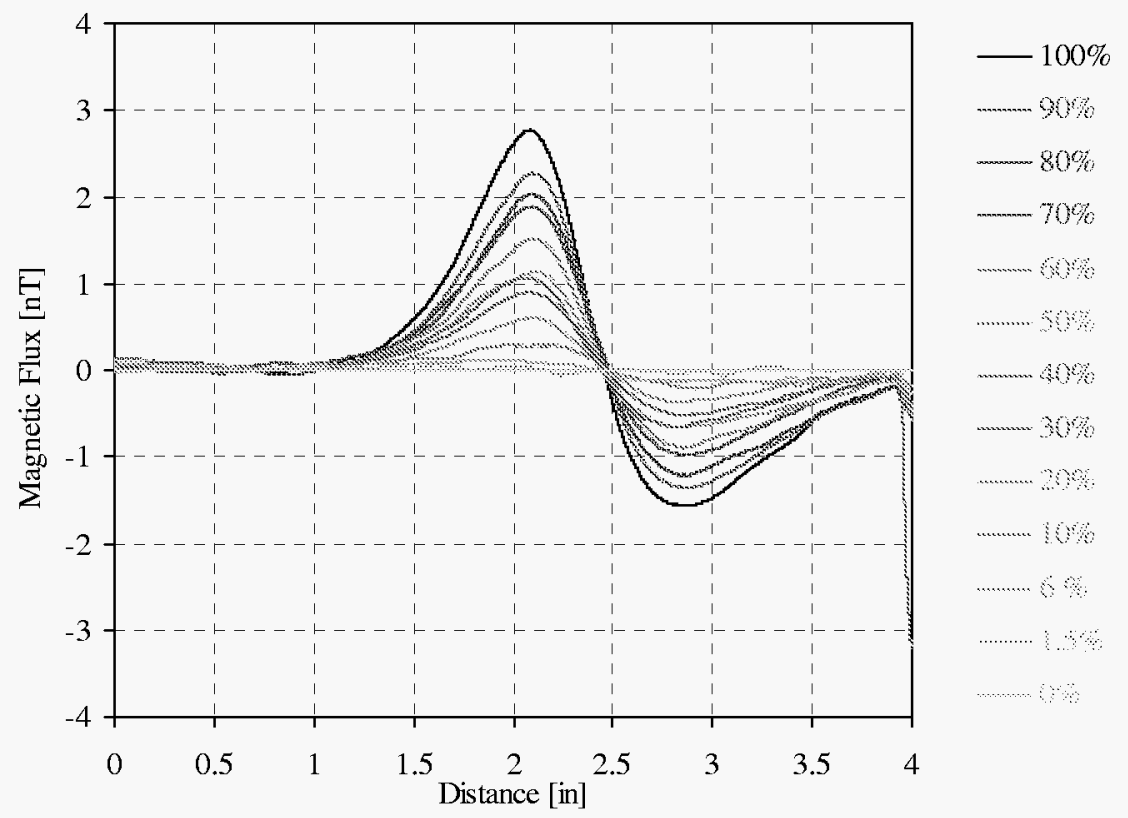

Figure 36 The net stress related thermoelectric magnetic signal produced under different stress ratios. 


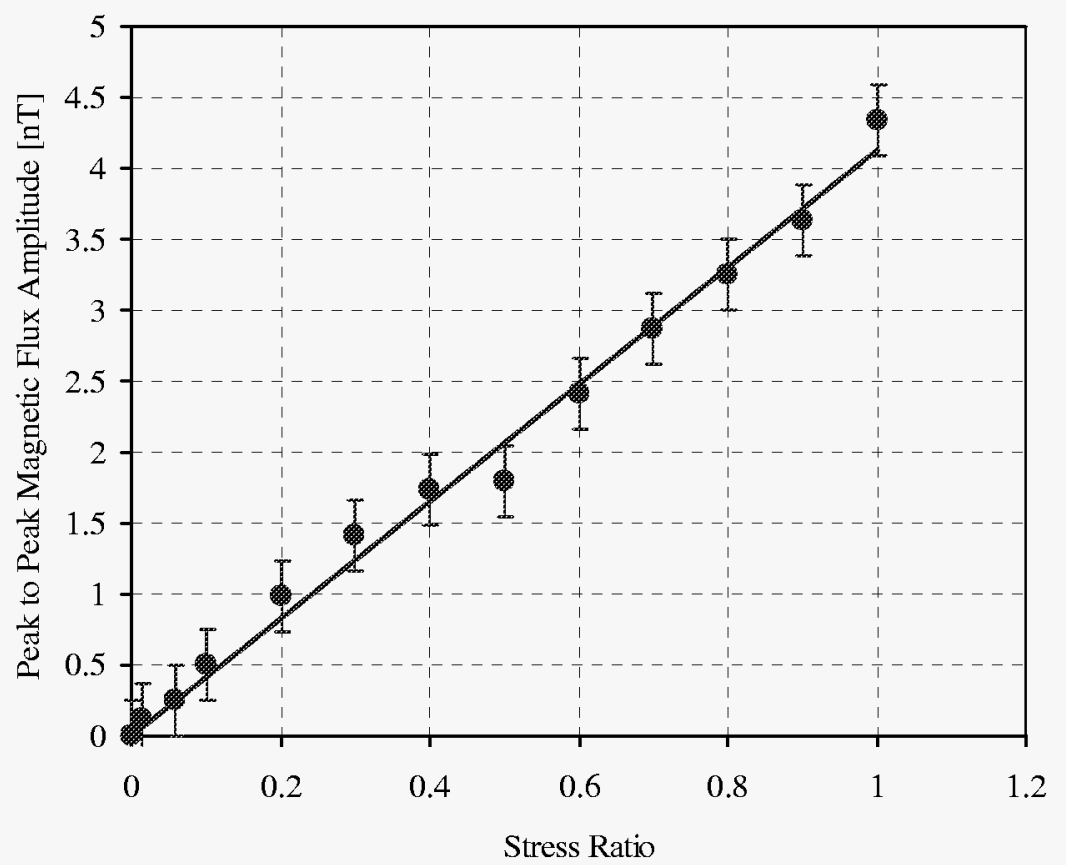

Figure 37 The peak to peak magnetic flux amplitude of the net stress related thermoelectric magnetic signature produced under different stress ratios.

These experiments illustrate the stress dependence of the thermoelectric magnetic signature. Quantitative evaluation of these signatures in terms of the stress dependence of the thermoelectric power can be done using the analytical model described in the previous chapter. This effort is still ongoing and after completion will be the topic of Bassam Abu-Nabah's MS thesis entitled "Thermoelectric detection of applied stresses in metals." However, the observation of the stress effect itself can be considered as a sign that the noncontacting thermoelectric technique can be exploited for residual stress assessment, an application of great practical importance that will be further investigated in the next chapter.

IX Thermoelectric signature produced by residual stresses

The noncontacting thermoelectric method can be used to characterize the prevailing residual stress in conducting specimens, which is usually produced by plastic deformation during either manufacturing or service. One particular application of great interest is the nondestructive assessment of residual stresses in surface-treated metals. It is well known that surface properties 
play a major role in determining the overall performance and, in particular, the fatigue resistance of structural components. Shot peening, one of the most popular surface improvement methods, induces compressive residual stresses in the surface layers of metallic parts via bombarding the them with a stream of high-velocity shots as it is schematically shown in Fig. 38. As the plastically deformed surface layer tries to expand relative to the intact interior of the specimen, compressive residual stress develops parallel to the surface at shallow depths, while beneath this layer a reaction-induced tensile stress results. Generally, the compressive stress at the surface is several times greater than the subsurface tensile stress. This near-surface compressive stress offsets any service-imposed tensile stress, retards fatigue crack nucleation and growth, and ultimately extends the fatigue life of the part. As Fig. 38 illustrates, in addition to the primary residual stress effect, shot peening also causes an adverse geometrical side effect by roughening the surface and certain relatively subtle variations in material properties, such as increased hardness and texture, that are consequences of the significant plastic deformation through cold work.

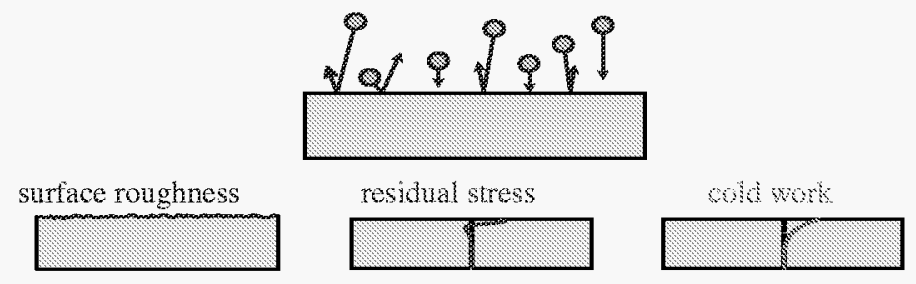

Figure 38 A schematic diagram of shot peening and its three major effects.

Residual stress analysis by nondestructive methods is a highly developed field using a great variety of different physical principles ranging from radiography, ultrasonics, electromagnetism, and ferromagnetism to assess the absolute level and relative distribution of elastic stresses prevailing in the material [24]. The most advanced technique for measuring residual stress in crystalline materials is based on X-ray diffraction (XRD), which measures changes in atomic inter-planar spacing to determine the magnitude of the prevailing elastic strain (stress). Neutrons can penetrate many millimeters into most engineering materials, while X-rays are typically absorbed within a surface layer of 5-20 $\mu \mathrm{m}$. In comparison, the crucial compressive part of typical residual stress profiles ranges from $50 \mu \mathrm{m}$ to $500 \mu \mathrm{m}$. Therefore, residual stress assessment by XRD is nondestructive only within a very shallow surface layer. To probe the 
residual stress below the surface, successive layers must be removed, usually through etching or electropolishing, i.e., in a destructive manner. The removal of material also alters the stress field, and thus requires theoretical corrections of the measured values. Furthermore, since the method probes only the surface, the results can be easily skewed by spurious effects in the extremely shallow top layer. In spite of the troublesome and destructive sectioning required by the low penetration depth, XRD is probably the most accurate and reliable method for residual stress assessment in surface-treated metals. One of the main reasons for this is that XRD methods are not significantly influenced by additional variations in material properties such as hardness, plastic strain, or texture.

In recent years, several candidates were identified for subsurface nondestructive stress evaluation including ultrasonic, eddy current, and other methods. For a long time, the characteristic dependence of ultrasonic surface wave velocity on stress has been thought to be very promising for residual stress measurements in surface-treated metals, though these expectations have remained largely unfulfilled as far as shot-peened specimens are concerned [25-27]. One of the main problems is that the most adverse side effect of shot peening, namely the rough surface topography, is also the most persistent. It is unaffected at best, or even exacerbated, by extended service during which the primary residual stress gradually relaxes. Eddy current conductivity measurements suffer from essentially the same limitation [28,29] except in the case of nickel-base superalloys [30,31], which is not surprising since eddy currents exhibit similar "surface hugging" behavior as Rayleigh-type surface waves. It was found that in surface wave velocity and electrical conductivity measurements the difference between lowstress-ground and shot-peened parts of the same specimen is essentially unaffected by annealing, which clearly indicates that the observed phenomenon is mainly due to surface roughness that increases the path length of surface-hugging Rayleigh waves and eddy currents.

Because of the diminishing contribution of the slowly fading residual stress effect during long-term service, any NDE method that is expected to reliably characterize the thermal relaxation process in shot-peened metals must be entirely insensitive to the persistent surface roughness effect. We have already demonstrated that the noncontacting thermoelectric technique is very sensitive to the presence of foreign body inclusions, when the thermoelectric power of the affected region is significantly different from that of the surrounding medium. The question arises whether mere plastic deformation of the material can produce a perceivable thermoelectric 
contrast with respect to the surrounding intact host. To answer this question, Fig. 39 compares the magnetic scans of two apparently similar 9.53-mm-diameter surface holes in copper. Figure 39a corresponds to a semi-spherical hole produced by low-stress milling which is expected to generate only negligible hardening and residual stress below the machined surface. In comparison, Fig. 39b shows the results from an otherwise similar semi-spherical indentation produced by pressing a stainless steel ball into the material in a manner that simulates a single impact during shot-peening. As a result of plastic deformation, the surrounding material below and around the indentation is substantially hardened and supports significant residual stresses. In order to demonstrate that the proposed thermoelectric method could readily detect thermally induced stress release, Figs. 39c and d show the magnetic images of the same two specimens after annealing in a vacuum furnace for 30 minutes at $700{ }^{\circ} \mathrm{C}$. All the effects of plastic deformation during indentation, as well as the much weaker manufacturing texture found in the original bar stock, are gone. As a result, the thermoelectric currents are also eliminated and the measured magnetic field is essentially zero.
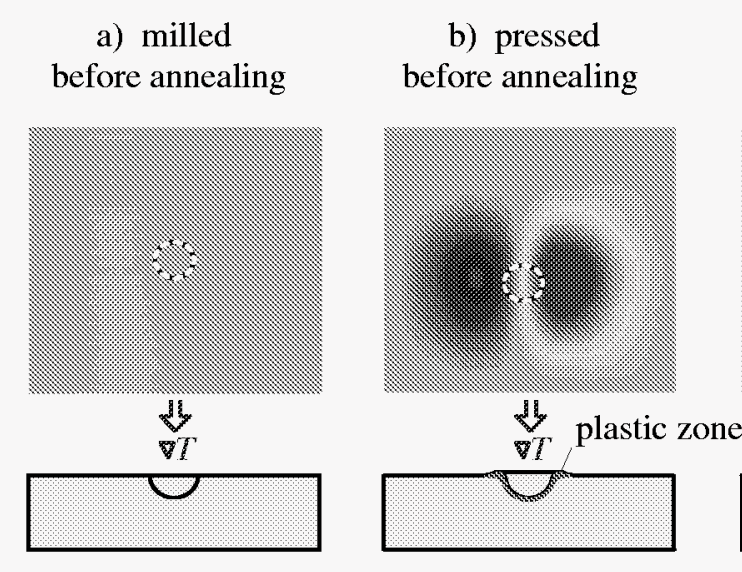

c) milled
after annealing
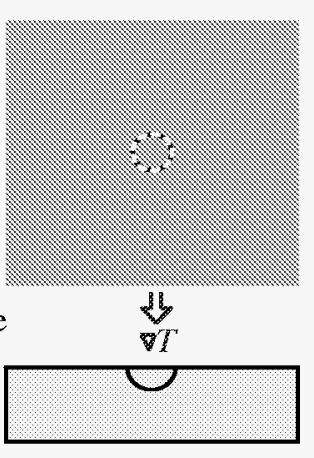

d) pressed after annealing

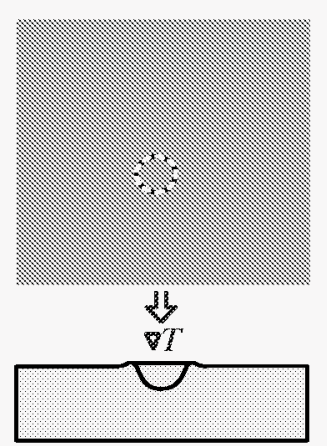

Figure 39 Comparison between (a) a semi-spherical hole produced by low-stress milling and (b) an otherwise similar semi-spherical indentation produced by pressing a stainless steel ball into the material. The scans on the right ( $\mathrm{c}$ and $\mathrm{d}$ ) were taken after annealing $\left(\nabla T \approx 0.5^{\circ} \mathrm{C} / \mathrm{cm}, 2 \mathrm{~mm}\right.$ lift-off distance, $\left.76.2 \mathrm{~mm} \times 76.2 \mathrm{~mm}\right)$. 


\section{IX.1 Monitoring residual stress relaxation in copper}

In sharp contrast with conventional ultrasonic surface wave velocity and eddy current conductivity measurement, the noncontacting thermoelectric technique is capable of monitoring thermo-mechanical stress relaxation in surface treated metals [16]. In order to further demonstrate this capability, we conducted a detailed experimental investigation of residual stress relaxation in shot-peened copper specimens. Figure 40 shows the schematic diagram of the noncontacting thermoelectric method as used for the characterization of shot-peened specimens. Since the generated magnetic field is perpendicular to both the heat flux in the specimen, which is parallel to the surface, and the gradient of the material property, which is normal to the surface, the magnetometer was polarized in the tangential direction relative to the surface.

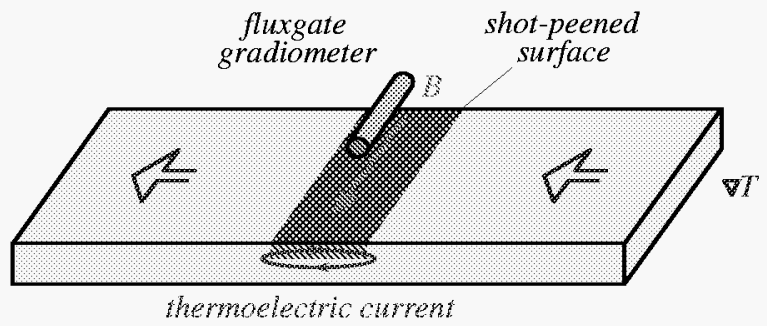

Figure 40 A schematic diagram of the noncontacting thermoelectric method as used for the characterization of shot-peened specimens.

On actual surface treated specimens the residual stress and cold work effects can be most easily reduced by appropriately chosen heat treatment that simulates thermally activated stress release during service. Figure 41 shows the measured Rockwell $\mathrm{F}$ hardness of cold-rolled C11000 copper as a function of annealing temperature in a vacuum furnace for 30 minutes. There is a sharp drop at around $450{ }^{\circ} \mathrm{C}$ where recrystallization occurs. Accordingly, we decided to conduct our first partial stress relaxation experiments at $315^{\circ} \mathrm{C}$. Figure 42 shows examples of the measured magnetic signatures on C11000 copper specimens before and after stress release for 30 minutes at $315{ }^{\circ} \mathrm{C}$ for five different peening intensity at 2-mm lift-off distance and 2.5 ${ }^{\circ} \mathrm{C} / \mathrm{cm}$ temperature gradient. On the intact specimens (i.e., before stress release) the peak-to-peak value of the measured magnetic flux density increased from $\approx 5 \mathrm{nT}$ to $\approx 20 \mathrm{nT}$ as the peening 
intensity increased from Almen 2 to Almen 16, while on the partially stress released specimens the magnetic flux density was approximately $75 \%$ lower.

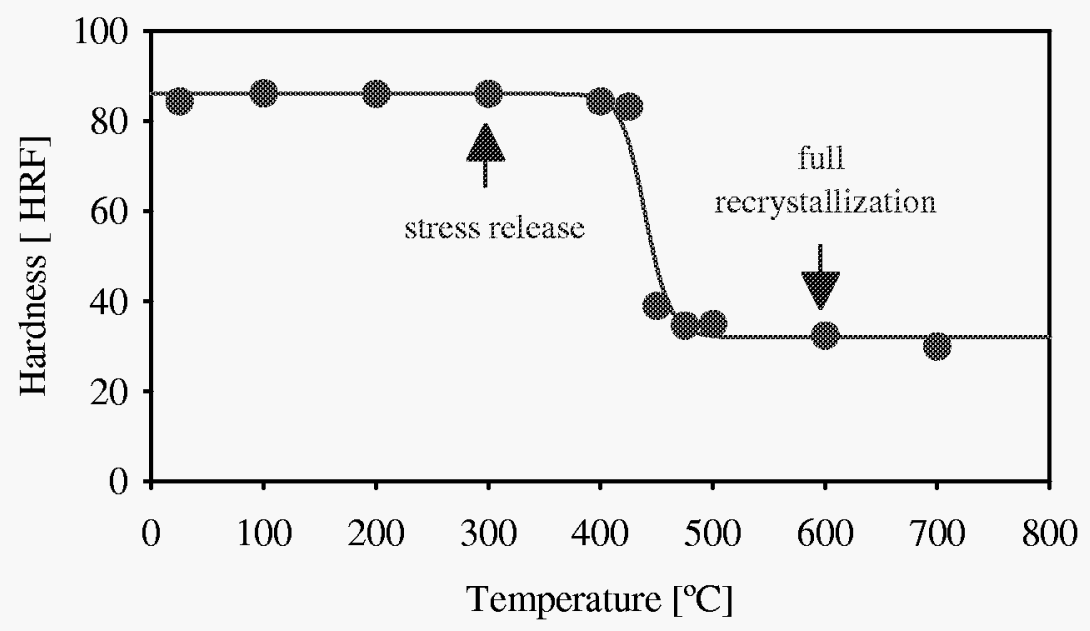

Figure 41 Rockwell F hardness versus annealing temperature in cold-rolled C11000 copper (in a vacuum furnace after 30 minutes).
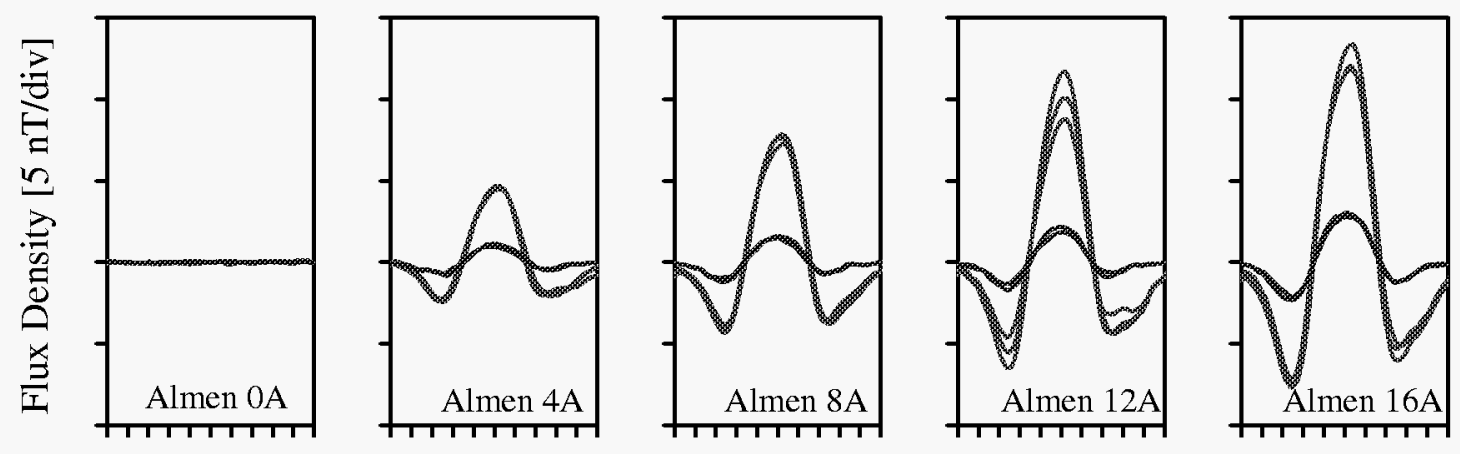

\section{Lateral Position $[10 \mathrm{~mm} / \mathrm{div}]$}

Figure 42 Magnetic signatures recorded on C11000 copper specimens before and after partial stress release for five different the shot peening intensities (red lines - as received after shot peening, blue lines - stress released for 30 minutes at $315^{\circ} \mathrm{C}$ ).

Figure 43 shows that the measured magnetic signature is more or less a linear function of the shot peening intensity and that this signature gradually decreases during relaxation to essentially zero in fully recrystallized specimens. These trends are very promising for the feasibility of nondestructive monitoring of thermal relaxation in shot-peened copper specimens, but they do not provide unequivocal evidence whether the magnetic signature is caused mainly 
by the presence of residual stresses, the presence of cold work, or a certain combination of both. In order to establish the relative role of the competing residual stress and cold work contributions in the measured thermoelectric signature, beside measuring the thermoelectric signature at different stages of thermal relaxation, we also monitored the decay of all relevant material properties such as residual stress, hardness, texture, and dislocation density using destructive micro-indentation and X-ray diffraction measurements. Figure 44 shows the thermal relaxation of the integrated residual stress, integrated cold work, and peak-to-peak magnetic signature in Copper $\mathrm{C} 11000$ for different Almen intensities. A statistical comparison of these integrated residual stress and cold work results to the magnitude of the decaying magnetic signature revealed that in shot-peened C11000 copper, on the average, approximately $64 \%$ of the thermoelectric signal is due to residual stresses and the remainder of the signal is caused by coldwork while the contribution of surface roughness is negligible.

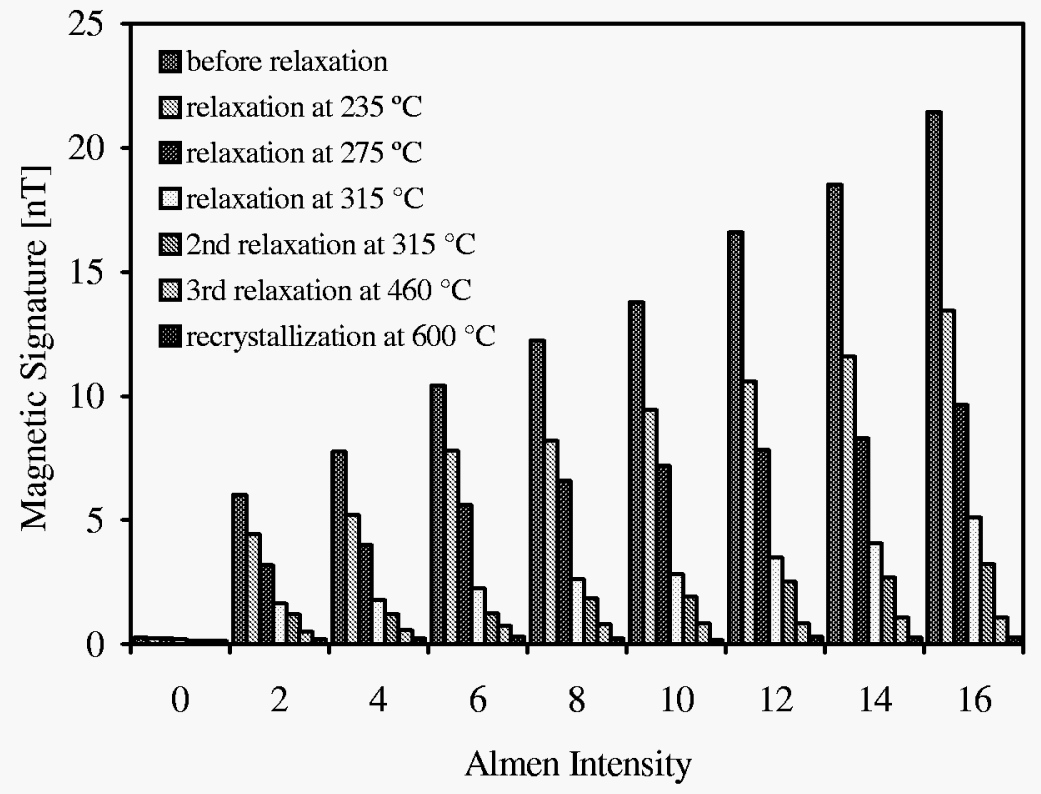

Figure 43 Average peak-to-peak amplitudes of the magnetic signatures recorded on C11000 copper specimens as functions of the shot peening intensity before and after partial stress release.

These experimental results clearly verify the feasibility of nondestructive evaluation of thermal relaxation in shot-peened $\mathrm{C} 11000$ copper. Although the development of a quantitative residual stress measurement method based on the noncontacting thermoelectric technique 
obviously requires additional more accurate and more detailed tests, these initial results are very promising. One of the most important questions to be addressed is whether the thermoelectric method is applicable to other engineering materials of special interest in fracture-critical applications. In particular, it is very important to develop NDE techniques for high-strength, high-temperature materials such as nickel-base superalloys, which exhibit much lower thermal and electrical conductivities, therefore will require more sensitive detection methods. Additional efforts in this direction will be discussed in the following section.

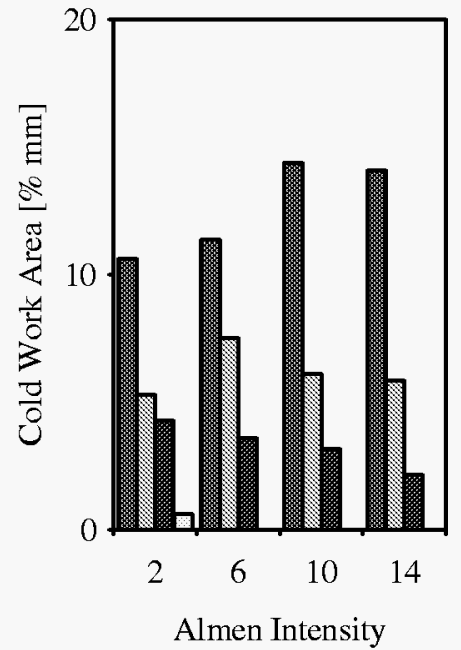

intact,
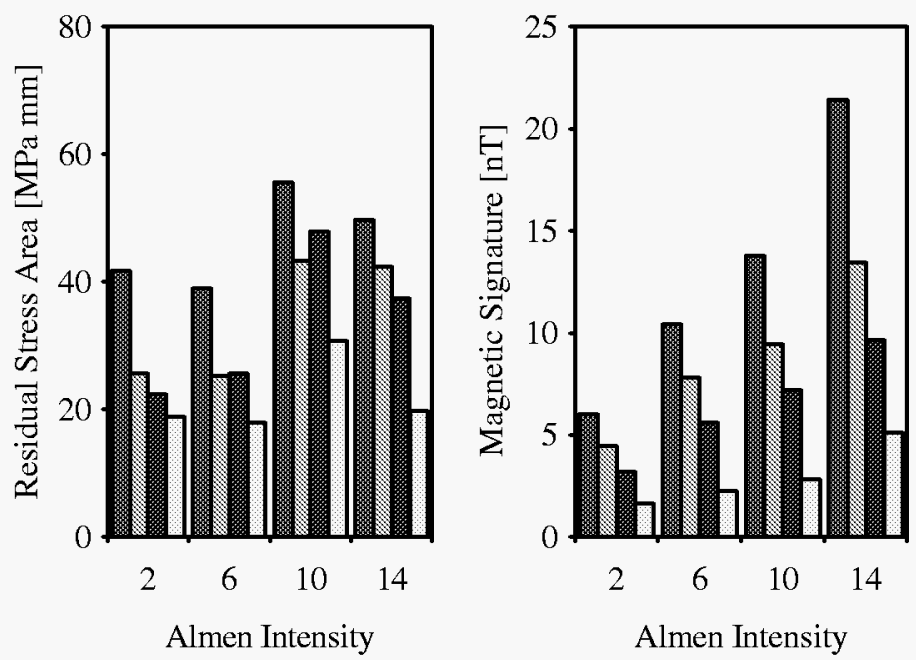

니 $235^{\circ} \mathrm{C}, \quad 275^{\circ} \mathrm{C}$, 回 $315^{\circ} \mathrm{C}$,

Figure 44 Thermal relaxation of the integrated residual stress, integrated cold work, and peak-to-peak magnetic signature in Copper C11000 for four different Almen intensities.

IX.2 Monitor ing residual stress relaxation in nickel-base superalloys

We have conducted similar noncontacting thermoelectric measurements in nickel-base superalloys to demonstrate that this method can be adapted to the characterization of the prevailing residual stress in shot-peened specimens of low electrical and thermal conductivity. These measurements were made on IN100 specimens both before and after partial stress relaxation. Like most high-temperature metals, nickel-base superalloys exhibit much lower thermal and electrical conductivity than single-phase pure copper, which necessitates certain 
modifications on the measurement system. In the simplest form of noncontacting thermoelectric NDE, a uniform temperature gradient is established throughout the specimen to be inspected by external heating and cooling. This can be achieved, for example, by enforcing appropriate boundary conditions, i.e., surface temperatures, far away from the inspected region. This approach might significantly simplify the analytical calculations, but it also renders them less relevant in certain types of measurements. Because of the inherently low spatial resolution of magnetic scanning, the flaw signal originating from a small area of the specimen must compete with the spurious magnetic signature emanating from the whole interior of the specimen, which is exposed to the temperature gradient. Much better detection sensitivity and resolution could be achieved if the temperature gradient were focused in the immediate vicinity of the region of interest, which is relatively easy to do in the case of surface or near-surface imperfections.

Figure 45 shows a photograph and a schematic diagram of a modified thermoelectric inspection system using noncontacting forced-air heating and cooling. In order to increase the temperature gradient in the region of interest we used a so-called vortex tube to produce highand low-temperature air streams that are directed at the specimen by nozzles placed on the two sides of the fluxgate. The vortex tube is balanced so that, at the air pressure used to drive it, the increase and decrease of the outgoing air temperature on the hot and cold sides are essentially the same. This mode of forced-air heating and cooling assures that significant temperature gradients arise only in the close vicinity of the surface and the rest of the specimen remains at room temperature. In this way, beside the obvious advantage of being noncontacting, this type of thermal excitation is much less sensitive to bulk inhomogeneity and anisotropy in the interior of the specimen, therefore it produces a lower background signal which could interfere with the useful signal of surface imperfections such as fretting damage.

This technique is much less sensitive to bulk inhomogeneity and anisotropy in the interior of the specimen, therefore it produces a lower background signal that could interfere with the useful signal produced by the affected surface layer. The magnetic signatures produced by the forced-air system at $\approx 30{ }^{\circ} \mathrm{C} / \mathrm{cm}$ temperature gradient were found to be similar to but slightly stronger than those obtained by the water-based system. Figure 46 shows the peak-topeak magnetic flux density as a function of the stress release time at $670{ }^{\circ} \mathrm{C}$ annealing temperature for shot-peened IN100 specimens. In spite of the considerable scatter in this 
preliminary data, a clear increase with peening intensity as well as a clear decay with increasing annealing time is apparent in the magnetic flux density.

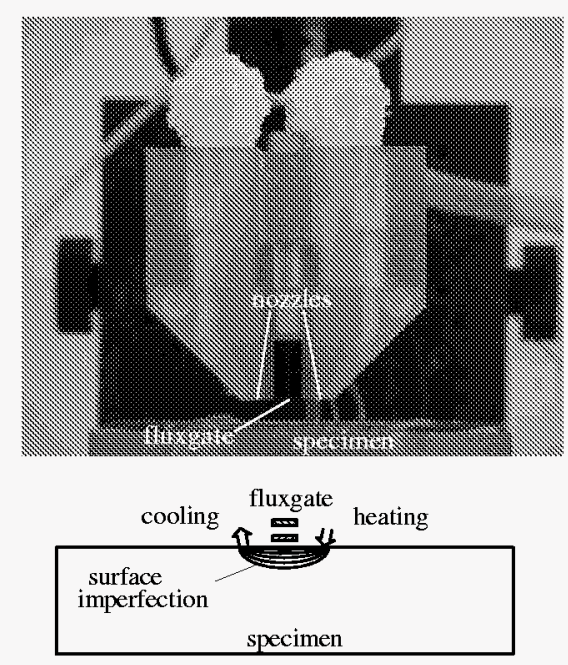

Figure 45 A photograph and a schematic diagram of the modified thermoelectric inspection system using noncontacting forced-air heating and cooling.

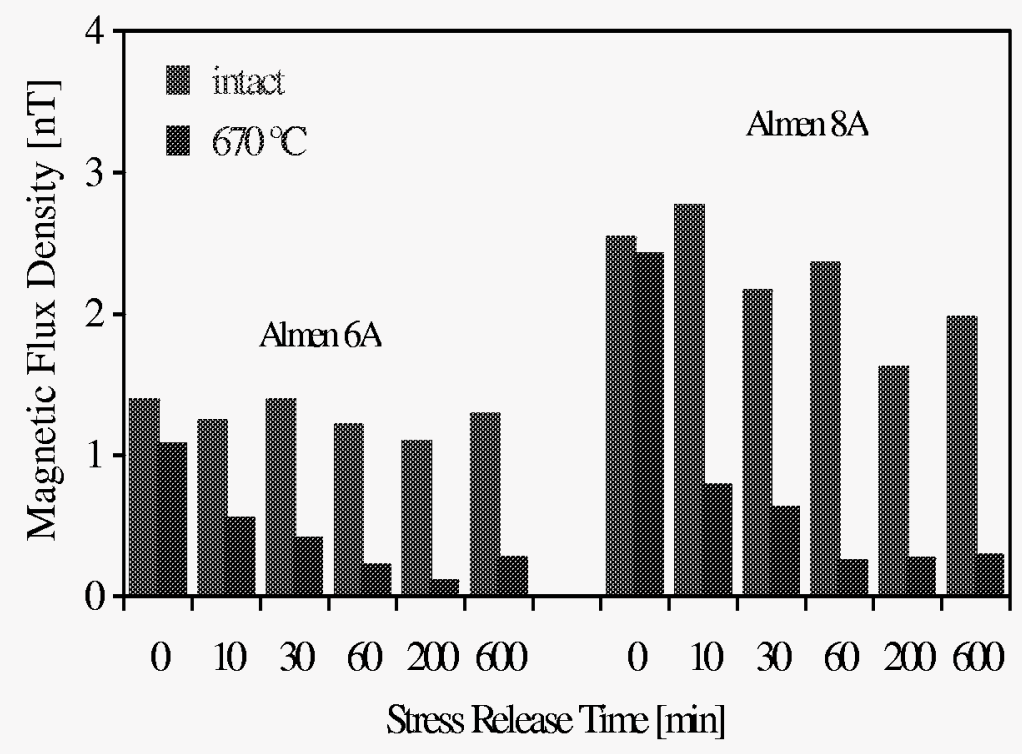

Figure 46 Peak-to-peak magnetic flux density versus stress release time at $670{ }^{\circ} \mathrm{C}$ in shotpeened IN100 specimens.

These experimental results illustrate the potential for a new noncontacting thermoelectric NDE technique to detect plastic deformation and the presence of residual stresses, which are very difficult to characterize by other, more conventional NDE methods. Beside the primary 
residual stress effect, the thermoelectric method is also sensitive to the secondary "material" effects of shot peening (local texture, increased dislocation density, hardening), but it is entirely insensitive to its "geometrical" by-product, i.e., the rough surface topography. Our initial experimental results indicate that unequivocal separation of residual stress relaxation from the parallel decay of secondary cold work effects is not feasible at this point and further research is needed to better understand the relationship of these two contrast mechanisms, especially in the case of low-conductivity materials like titanium alloys and nickel-base superalloys.

Despite the considerable complexity of the problem, our results indicate that nondestructive evaluation of near surface material variations in surface-treated metals is feasible, though additional research is needed to further optimize this promising NDE method. It is expected that the noncontacting thermoelectric technique could be also used for the characterization of different surface-treated metals, including laser shock peened and lowplasticity burnished components. This nondestructive inspection technique is aimed primarily at quantitatively assessing the degree of stress release during long-term service at elevated temperatures. This goal can be achieved by measuring the thermoelectric signature that is related to the weighted average of the compressive residual stress below the surface. This limited singleparameter characterization is acceptable since the residual stress profile is primarily determined by material and manufacturing process variables and will not change significantly its shape or average depth during thermally activated stress release except for the well-known accelerated near-surface relaxation due to the presence of cold work. Further work is needed to verify the feasibility of this technique by destructive profiling of the residual stress in specimens at different stages of thermal stress release using X-ray diffraction measurements. Although it is expected that the thermoelectric method will be found to be sensitive to secondary cold work effects as well as to primary residual stress effects, it still can be a very useful NDE tool since quantitative assessment of the level and distribution of cold work in surface-treated metals is of primary importance from the point of view of thermo-mechanical stability of the beneficial residual stresses. 
$\mathrm{X} \quad$ Thermoelectric signals produced by material embrittlement

The excellent sensitivity of the new noncontacting thermoelectric method can be also exploited to address crucial NDE issues such as the detection of High-Interstitial Defects in titanium alloys, which has been the focus of attention since the application of titanium to rotating components in gas turbines. Probably the best known such defects are hard-alpha inclusions, which are low-density, brittle regions of spuriously high nitrogen and oxygen content, that occur in titanium alloys. These inclusions are often cracked or include voids, which makes it much easier to detect them by conventional NDT methods including ultrasonic backscattering. However, the detection of uncracked hard-alpha inclusions represents a very significant challenge for NDT. In this project, we also investigated the feasibility of thermoelectric detection of hard-alpha inclusions in titanium.

Since real hard-alpha specimens are extremely hard to come by, we used TitaniumNitride coupons of different Nitrogen content (courtesy of Mike Gigliotti of GE CRC). Figure 47a shows the absolute thermoelectric power of five TiN coupons compared to that of Ti-6Al-4V (blue reference line). We also measured the thermoelectric signatures of four 0.1"-diameter 0.2"long TiN inclusions made by powder-metallurgy, which were then embedded in a Ti-6Al-4V host using diffusion bonding. Figure $47 \mathrm{~b}$ shows the peak-to-peak magnetic signatures produced by these inclusions just below the surface. Although the Nitrogen contents of the large coupons and the small embedded nuggets were not exactly the same, the observed trends are fairly similar, which indicates that the produced thermoelectric contrast could well correlate with the nitrogen content in hard-alpha inclusions as well. It should be mentioned that the spurious material signature of the textured $\mathrm{T}-6 \mathrm{Al}-4 \mathrm{~V}$ host was between 5-20 nT depending on the orientation of the specimen, therefore we used the previously described rotation technique to eliminate its adverse effect. In particular, we measured the magnetic signals at four different orientations rotated with respect to each other by $90^{\circ}$ in each step and averaged the results. This is a rather cumbersome, but highly efficient method to eliminate both the inhomogeneous and anisotropic material signatures, which otherwise would effectively hide the presence of the inclusion. 

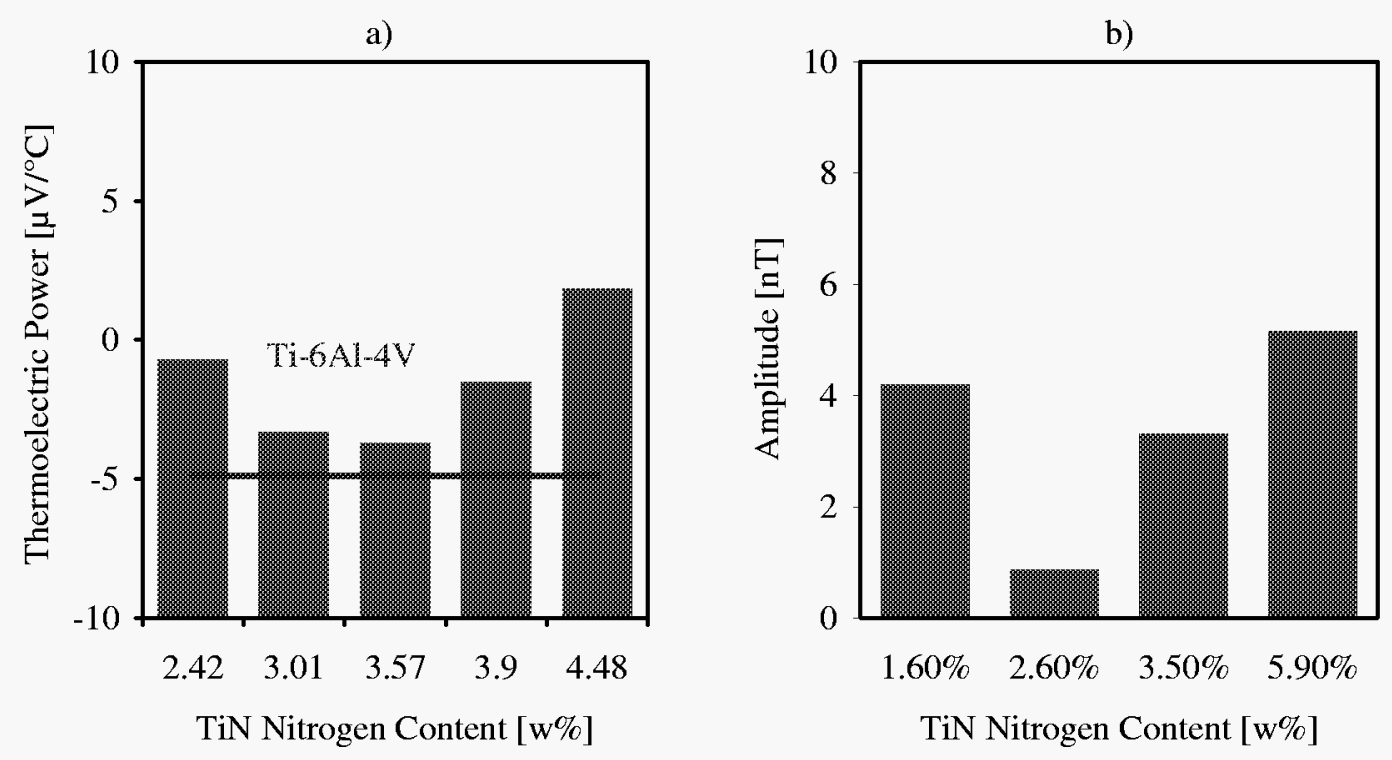

Figure 47 Absolute thermoelectric power of five TiN coupons (a) and the peak-to-peak magnetic signatures produced by four TiN inclusions in a Ti-6Al-4V host (b).

\section{Conclusions}

This project was aimed at developing a new noncontacting thermoelectric method for nondestructive detection of material imperfections in metals. The method is based on magnetic sensing of local thermoelectric currents around imperfections when a temperature gradient is established throughout a conducting specimen by external heating and cooling. The surrounding intact material serves as the reference electrode therefore the detection sensitivity could be very high if a sufficiently sensitive magnetometer is used in the measurements. This self-referencing, noncontacting, nondestructive inspection technique offers the following distinct advantages over conventional methods: high sensitivity to subtle variations in material properties, unique insensitivity to the size, shape, and other geometrical features of the specimen, noncontacting nature with a substantial stand-off distance, and the ability to probe relatively deep into the material.

Our experimental results indicate that spatially incoherent material noise produced by microstructural features (e.g., grains, second phases, or precipitations) exert a negligible effect on noncontacting thermoelectric inspection because of the low spatial resolution of the 
measurement. Therefore, our efforts were focused on the investigation of the spatially coherent background signature produced by macrostructural features (e.g., heat-affected, work-hardened, textured, or stressed regions). This spurious background signature is called material noise only because it interferes with, and often conceals, the flaw signal to be detected and ultimately determines the achievable probability of detection (POD) of a given flaw, although in certain applications it can be used as a useful thermoelectric signal to characterize the intact material itself. Many manufacturing processes produce hardened, textured, recrystallized, or otherwise modified surface layers that often sustain significant levels of residual stresses. As a result, the material properties in the affected skin region are perceivably different from those of the intact material in the interior of the specimen.

We have established that the noncontacting thermoelectric method is uniquely sensitive to subtle variations in material properties and that it is essentially insensitive to geometrical features such as surface curvature and roughness. The potential applications of this method cover a very wide range from detection metallic inclusions and segregations, inhomogeneities, and tight cracks to characterization of hardening, embrittlement, fatigue, texture, and residual stresses. In this project, we laid down the foundations of a new field of nondestructive materials characterization and made substantial advance towards all of the originally proposed research goals. We have successfully adapted the noncontacting thermoelectric method to a series of nondestructive materials characterization applications that are currently not accessible by any other known inspection method. In particular, we developed and experimentally verified a series of analytical models capable of predicting the thermoelectric signatures produced by inclusions of different material properties and geometrical features. In addition, we studied the feasibility of nondestructive detection and characterization of cracks and voids in textured polycrystalline materials, phase anomalies and anisotropic effects of the microstructure, and thermally induced residual stress relief in surface treated components. Building on the extensive and very promising results of this project, it will be possible to develop numerous new NDT techniques that will find application in energy production (nuclear, oil, and gas industries, power generators, etc.), material manufacturing (aluminum, steel, titanium, nickel-base super-alloys, etc.), and in the transportation industry (aerospace, automobile, etc.). 


\section{References}

[1] Henry, E. B., Stuart, C. M., and Tomasulo, W., in Nondestructive Testing Handbook, Vol. 9 (ASNT, Columbus, 1995) pp. 363-376.

[2] Stuart, C. M., J. Testing Eval. 15, 224 (1987).

[3] Morgner W., Mat. Eval. 9, 1081 (1991).

[4] Hu, J., and Nagy, P. B., Appl. Phys. Lett. 73, 467 (1998).

[5] Nagy, P. B., and Hu, J., in Rev. Prog. Quant. NDE, Vol. 17 (Plenum, New York, 1998) pp. 1573-1580.

[6] Hinken, J. H., and Tavrin Y., in Rev. Prog. Quant. NDE, Vol. 19 (AIP, Melville, 2000) pp. 2085-2092.

[7] Maslov, K., and Kinra, V. K., Mat. Eval. 59, 1081 (2001).

[8] Tavrin, Y., Krivoy, G. S., Hinken, J. H., and Kallmeyer, J. P., in Rev. Prog. Quant. NDE, Vol. 20 (AIP, Melville, 2001) pp. 1710-1716.

[9] Carreon, H., Nagy, P. B., in Proc. 7th ASME NDE Topical Conf., Vol. 20 (ASME, New York, 2001) pp. 209-215.

[10] Nagy, P. B., and Nayfeh, A. H., J. Appl. Phys. 87, 7481 (2000).

[11] Carreon, H., Nagy, P. B., and Nayfeh, A. H., J. Appl. Phys. 88, 6495 (2000).

[12] Nayfeh, A. H., and Faidi, W. I., Eur. Phys. J. - Appl. Phys. 19, 153 (2002).

[13] Nayfeh, A. H., Carreon, H., and Nagy, P. B., J. Appl. Phys. 91, 225 (2002).

[14] A. H. Nayfeh, W. I. Faidi, and M. I. Jaghoub, "The role of anisotropy in the thermoelectric detection of holes in metals," European Physical Journal - Applied Physics 22, 103-109 (2003).

[15] Carreon, H., Lakshminarayan, B., Faidi, W. I., Nayfeh, A. H., and Nagy, P. B., "On the role of material property gradients in noncontacting thermoelectric NDE," NDT\&E International 36,339 (2003).

[16] Carreon, H., Nagy, P. B., and Blodgett, M. P., Res. Nondestr. Eval. 14, 59 (2002).

[17] Nye, J. F., Physical Properties of Crystals, Their Representation by Tensors and Matrices (Clarendon Press, Oxford, 1985).

[18] Carslaw, H. S. and Jaeger, J. C. , Conduction of Heat in Solids (Clarendon, Oxford, 1959).

[19] Livens, G. H., Theory of Electricity (The University Press, Cambridge, 1926). 
[20] Besant, W. H. and Ramsey, A. S., Hydrodynamics (G. Bell and Sons, London, 1920).

[21] Kropschot, R. H., and Blatt, F. J., Phys. Rev. 116, 617 (1959).

[22] Rowe, V. A., and Schroeder, P. A., J. Phys. Chem. Sol. 31, 1 (1970).

[23] Blodgett, M., and Nagy, P. B., Appl. Phys. Lett. 72, 1045 (1998).

[24] Hauk, V., Structural and Residual Stress Analysis by Nondestructive Methods (Elsevier, Amsterdam, 1997).

[25] Thompson, R. B., Lu, W. Y., and Clark, A. V. Jr., in Handbook of Measurement of Residual Stress (Fairmont Press, Lilburn, 1996) pp. 149-178.

[26] Lavrentyev, A. I., Stucky, P. A., and Veronesi, W. A.,. in Rev. Prog. Quant. NDE, Vol. 19 (AIP, Melville, 2000) pp. 1621-1628.

[27] Glorieux, C., Gao, W., Kruger, S. E., Rostyne, K. V., Lauriks, W, and Thoen, J., J. Appl. Phys. 88, 4394 (2000).

[28] Schoenig, F. C., and Soules, J. A., Chang, H., and DiCillo, J. J., Mat. Eval. 53, 22 (1995).

[29] Chang, H., Schoenig, F. C., and Soules, J. A., Mat. Eval. 57, 1257 (1999).

[30] Blodgett, M. P., and Nagy, P. B., J. Nondestr. Eval. 23, 107. (2004).

[31] Yu, F., and Nagy, P. B., J. Appl. Phys. 96, 1257 (2004).

\section{Achievements}

Published Refereed Journal Papers

P. B. Nagy and A. H. Nayfeh, "On the thermoelectric magnetic field of spherical and cylindrical inclusions," Journal of Applied Physics 87, 7481-7490 (2000).

H. Carreon, P. B. Nagy, and A. H. Nayfeh, “Thermoelectric detection of spherical tin inclusions in copper by magnetic sensing," Journal of Applied Physics 88, 6495-6500 (2000).

A. H. Nayfeh, H. Carreon, and P. B. Nagy, "Role of anisotropy in noncontacting thermoelectric materials characterization," Journal of Applied Physics 91, 225-231 (2002).

A. H. Nayfeh and M. Al Jaghoub, "Thermoelectrically induced magnetic fields in laminated composite metals," European Physical Journal - Applied Physics 18, 79-87 (2002). 
A. H. Nayfeh and W. I. Faidi, "Thermoelectrically induced magnetic field of elliptical cylindrical and spheroidal inclusions," European Physical Journal - Applied Physics 19, 153-164 (2002).

H. Carreon, P. B. Nagy, and M. P. Blodgett, "Thermoelectric Nondestructive Evaluation of Residual Stress in Shot-Peened Metals," Research in Nondestructive Evaluation 14, 59-80 (2002).

A. H. Nayfeh, W. I. Faidi, and M. I. Jaghoub, "The role of anisotropy in the thermoelectric detection of holes in metals," European Physical Journal - Applied Physics 22, 103-109 (2003).

H. Carreon, B. Lakshminarayan, W. I. Faidi, A. H. Nayfeh, and P. B. Nagy, "On the role of material property gradients in noncontacting thermoelectric NDE," NDT\&E International 36, 339-348 (2003).

Proceeding papers

A. H. Nayfeh, P. B. Nagy, and H. Carreon, "On the exploitation of thermoelectric coupling for characterization of inclusions in metals," in Nondestructive Evaluation and Characterization of Engineering Materials for Reliability and Durability Predictions, AMD-Vol 240/NDEVol 18 (ASME, New York, 2000) pp. 71-82.

H. Carreon and P. B. Nagy, "Characterization of shot-peened surfaces by a noncontacting thermoelectric method," in Nondestructive Evaluation and Characterization of Engineering Materials for Reliability and Durability Predictions, AMD-Vol 240/NDE-Vol 18 (ASME, New York, 2000) pp. 31-39

H. Carreon, P. B. Nagy, and A. H. Nayfeh, "Noncontacting thermoelectric detection of spherical inclusions in copper by magnetic sensing," in Review of Progress in Quantitative Nondestructive Evaluation Vol. 20 (AIP, New York, 2001) pp. 1694-1701.

A. H. Nayfeh, H. Carreon, and P. B. Nagy, "On the exploitation of thermoelectric coupling for characterization of inclusions in metals," in Review of Progress in Quantitative Nondestructive Evaluation Vol. 20 (AIP, New York, 2001) pp. 1686-1693.

H. Carreon and P. B. Nagy, "Detection of surface imperfections in metals by a noncontacting thermoelectric method," in Proceedings of the 7th ASME NDE Topical Conference, NDEVol. 20 (ASME, New York, 2001) pp. 209-215. 
H. Carreon, A. H. Nayfeh, and P. B. Nagy, "Anisotropic effects in noncontacting thermoelectric material characterization," in Review of Progress in Quantitative Nondestructive Evaluation Vol. 21 (AIP, New York, 2002) pp. 1455-1462.

H. Carreon, P. B. Nagy, and M. Blodgett, "Thermoelectric nondestructive evaluation of residual stress in shot-peened metals," in Review of Progress in Quantitative Nondestructive Evaluation Vol. 21 (AIP, New York, 2002) pp. 1667-1674.

B. Lakshminarayan, H. Carreon, and P. B. Nagy, "Monitoring of the level of residual stress in surface-treated specimens by a noncontacting thermoelectric technique," in Review of Progress in Quantitative Nondestructive Evaluation Vol. 22 (AIP, New York, 2003) pp. $1523-1528$.

H. Carreon, B. Lakshminarayan, and P. B. Nagy, "Thermoelectric material signature due to the presence of property gradients," in Review of Progress in Quantitative Nondestructive Evaluation Vol. 23 (AIP, New York, 2004) pp. 445-452.

Ph. D. Dissertations

W. I. Faidi, "Theoretical investigations of the thermoelectrically induced magnetic field in materials characterization," (February, 2002).

H. Carreon, "Detection of surface imperfections in metals by a noncontacting thermoelectric method," (June, 2002).

\section{S. Theses}

B. Lakshminarayan, "Experimental investigation of the thermoelectric background signature in Titanium- and Nickel-base alloys," (June, 2003).

F. Yu, "Thermoelectric detection of fretting fatigue damage," (January 2004).

B. Abu-Nabah "Thermoelectric detection of applied stresses in metals," (in progress) 